Aus der Klinik für Strahlenheilkunde

der Medizinischen Fakultät Charité

der Humboldt-Universität zu Berlin

\title{
HABILITATION
}

\section{Methoden des Prozessmanagements in der Radiologie}

\author{
Zur Erlangung des akademischen Grades \\ Venia Legendi \\ Für das Fach \\ Diagnostische Radiologie
}

\begin{abstract}
vorgelegt der Medizinischen Fakultät Charité der Humboldt-Universität zu Berlin
\end{abstract}

von

Ulf Teichgräber

aus Berlin 
Dekane: Prof. Dr. med. Joachim W. Dudenhausen

Prof Dr. Martin Paul

Gutachter: 1. Prof. Dr. med. Manfred Thelen

2. Prof. Dr. med. Mathias Langer

Datum der Habilitation: $\quad$ 04.12.2003 


\title{
Zusammenfassung
}

Innerhalb eines Krankenhauses beeinflusst der Radiologe in einem Verbund von Fachabteilungen wesentlich die Liegezeiten von Patienten durch die diagnostische Information und die Verfügbarkeit seiner Dienstleistungen. Die Organisation und zeitliche Bemessung von radiologischen Untersuchungsabläufen werden dadurch um so wichtiger. Der Arzt wird im Rahmen seines Studiums und seiner Facharztweiterbildung nicht im Organisationsmanagement ausgebildet, entsprechendes Fachwissen muss er sich in der Regel in Eigenregie aus Fachliteratur und Kursen aneignen. Trotzdem wird vom Arzt über die medizinische Versorgung hinaus zunehmend verlangt, dass es seinen Arbeitsabläufe auch unter wirtschaftlichen Gesichtspunkten optimiert. In dieser Arbeit werden verschiedene Methoden des Prozessmanagements für ihre Anwendung in der Radiologie erprobt. Unter Zuhilfenahme von einfachen papierbasierten Methoden lässt sich bereits ein Großteil von Arbeitsprozessanalysen durchführen. Bei komplexen Arbeitsabläufen wird man eine Methode wählen, die möglichst genaue qualitative und quantitative Aussagen über zu erwartende Effekte liefert. Vorgestellt werden diesbezüglich die Netzplantechnik und die Prozesssimulation anhand des computertomographischen und sonographischen Arbeitsplatzes.

\author{
Prozessmanagement \\ Prozesssimulation \\ Netzplantechnik \\ Kritischer Pfad \\ CQI Continuous Quality Improvement
}

\begin{abstract}
Within a hospital, the radiologist considerably influences the patient's length of stay through the availability of service and diagnostic information. Therefore, coordination and timing radiologic examinations become increasingly more important. Physicians are not taught organizational management during their medical education and residency training, and the necessary expertise in economics is generally acquired through literature or specialized courses. Beyond the medical service, the physicians are increasingly required to optimise their work flow according to economic factors. This review introduces various tools for process management and its application in radiology. By means of simple paper-based methods, the work flow of most processes can be analysed. For more complex work flow, it is suggested to choose a method that allows for an exact qualitative and quantitative prediction of the effect of variations. This review introduces network planning technique and process simulation.
\end{abstract}

\author{
Process management \\ Process simulation \\ Network planning technique \\ Critical Pathway method \\ CQI Continuous quality improvement
}




\section{Inhaltsverzeichnis}

1.1 Betrachtung der Arbeitsprozesse im Krankenhaus

1.2 OPtIMIERUNG EINES ARBEITSPROZESSES

1.3 Methodik des Prozessmanagements 11

1.3.1 NETZPLANTECHNIK 12

1.3.1.1 Metra-Potential-Methode (MPM): $\quad 12$

1.3.1.2 Program Evaluation and Review Technique (PERT): 13

1.3.2 PROZESSSIMULATION 17

$\underline{2}$ ZIELSETZUNG 19

$\underline{3}$ ARBEITSPROZESSANALYSE FÜR DEN EINSATZ DER MOBILEN 20

3.1 COMPUTERTOMOGRAPHIE AUF DER INTENSIVSTATION 20

3.1.1 EINLEITUNG 20

3.1.1.1 Mobile Diagnostik 20

3.1.2 MATERIAL UND METHODIK:

3.1.2.1 Einsatzorte 23

3.1.3 ERGEBNISSE: $\quad 25$

3.1.3.1 Zeitnahme $\quad 25$

3.1.3.2 Korrelation der Entfernung und der Zeitmessung 28

3.1.3.3 Evaluation des intensivmedizinischen Personals 29

\begin{tabular}{ll}
3.1 .4 & DISKUSSION \\
\hline
\end{tabular}

4 ANWENDUNGEN DER NETZPLANTECHNIK

4.1 NetZPLANTECHNIK ZUR ZEITPLANUNG BEI ABDOMINELLEN

ULTRASCHALLUNTERSUCHUNGEN

4.1.1 EINLEITUNG

4.1.2 METHODE $\quad 35$

4.1.3 ERGEBNISSE 38

4.1.3.1 Phase der Strukturplanung 39

4.1.4 DISKUSSION 42

4.2 ANWENDUNG DER NETZPLANTECHNIK FÜR DIE ARBEITSPROZESSANALYSE IM
KLINISCHEN BEREICH

4.2.1 ZiELSETZUNG: QuAlität UNd EfFIZIENZ IM ROUTINEBEREICH

4.2.2 MATERIAL UND METHODIK $\quad 47$

4.2.3 BESCHREIBUNG DES BETRACHTETEN ARBEITSPLATZES 48

4.2.4 BENCHMARKING VON ARBEITSPROZESSEN 49

4.2.5 EINFÜHRUNG IN DIE NETZPLANTECHNIK 49

4.2.6 DEFINITION EINES NETZPLANS

4.2.7 AKTIVITÄTEN/VORGÄNGE

$\begin{array}{lll}4.2 .8 \text {.RESSOURCENPLANUNG } & 52\end{array}$ 
4.2.9 ANORDNUNGSBEZIEHUNGEN 52

4.2.9.1 Darstellungsformen in der Netzplantechnik 53

4.2.9.2 Vorgangsorientierter Netzplan $\quad 53$

4.2.9.3 Varianten der Netzplantechnik 54

4.2.9.4 Verfahren für deterministische Projektabläufe 56

4.2.9.5 Metra Potential Method $\quad 57$

4.2.9.6 Verfahren für stochastische Projektabläufe 58

4.2.9.7 Vergleich der Verfahren $\quad 59$

4.2.9.8 Ressourcenplanung $\quad 59$

4.2.10 SOFTWARE $\quad 60$

4.2.11 ANFERTIGUNG EINES NETZPLANS $\quad 60$

4.2.11.1 Phase I: Strukturermittlung 60

4.2.11.2 Phase II: Messdatenerfassung $\quad 62$

4.2.11.3 Phase III: Netzplan anfertigen 63

4.2.11.4 Phase IV: Auswertung 63

4.2.12 ERgEBNISSE: NETZPLAN FÜR DEN ULTRASCHALL ROUTINEARBEITSPLATZ 65

$\begin{array}{lll}4.2 .13 & \text { DISKUSSION } & 67\end{array}$

4.2.14 SCHLUSSFOLGERUNGEN

4.3 ANWENdung DeR NetZPLANTECHNIK In DER COMPUTERTOMOgRAPHIE

4.3.1 EINLEITUNG

4.3.1.1 Patientenorientierte Optimierung der Arbeitsprozesse $\quad 71$

4.3.1.2 Netzplantechnik $\quad 74$

4.3.1.3 Erfahrungen mit CPM und PERT in der klinischen Medizin 74

4.3.2 FRAGESTELLUNG

4.3.3 METHODIK $\quad 77$

4.3.3.1 Beschreibung der CT-Arbeitsplätze $\quad 77$

$\begin{array}{ll}\text { 4.3.3.2 Patientenkategorien } & 78\end{array}$

$\begin{array}{ll}\text { 4.3.3.3 Untersuchungskategorien } & 78\end{array}$

4.3.3.4 Erstellen des Prozessmodells $\quad 78$

4.3.3.5 Auswertung $\quad 85$

4.3.4 ERGEBNISSE $\quad 85$

4.3.4.1 Messdatenerfassung $\quad 85$

4.3.4.2 Die CT-Untersuchung wurde in drei Abschritten getrennt betrachtet: $\quad 86$

4.3.4.3 Erhobene Messdatenmenge $\quad 89$

4.3.4.4 Wahrscheinlichkeitsverteilung 89

4.3.4.5 Bestimmung der Zeitreserven und Optimierung des Netzplanes 94

4.3.5 PUFFERZEITEN $\quad 95$

$\begin{array}{ll}\text { 4.3.5.1 Auswertung } & 97\end{array}$

4.3.5.2 Auswertung von unterschiedlichen Kategorien innerhalb einer gleichen Untersuchungsart 100

4.3.6 DISKUSSION 102

4.3.6.1 Zeitmanagement 102

4.3.6.2 Verkürzung der Projektdauer unter Verwendung von CPM 103

4.3.6.3 Verringerung der Ausführungsdauer von kritischen Vorgängen 104

4.3.6.4 Parallele Durchführung der Vorgänge 105

4.3.6.5 Änderung der Ablaufstruktur 105

$\begin{array}{ll}\text { 4.3.6.6 Pufferzeiten } & 105\end{array}$

4.3.6.7 Reduktion der Varianz / Verwendung von PERT 106

4.3.6.8 Durchführungszeiten in unterschiedlichen Patientenkategorien 107

$\begin{array}{ll}\text { 4.3.6.9 Vorschläge zur Optimierung der Arbeitsabläufe im } & \\ \text { computertomographischen Arbeitsbereich } & 108\end{array}$ 


\section{$\underline{5}$ EFFIZIENZSTEIGERUNG DURCH EINE DIGITALE INFRASTRUKTUR AN}

EINEM ROUTINEULTRASCHALLARBEITSPLATZ- EINE QUANTITATIVE ABSCHÄTZUNG MITTELS PROZESSSIMULATION

5.1 EINLEITUNG

5.2 Material und Methoden

5.2.1 BESCHREIBUNG DES UNTERSUCHTEN ULTRASCHALLARBEITSPLATZES

5.2.2 DIGITALE INFRASTRUKTUR

5.2.3 PROZESSSIMULATION

5.2.3.1 Phase I

5.2.3.2 Phase II: Strukturermittlung

5.2.3.3 Phase III: Messdatenerfassung

5.2.3.4 Phase IV: Prozessmodell anfertigen

5.2.3.5 Phase V: Auswertung

6.1 LITERATUR

6.2 ABKÜRZUNGSVERZEICHNIS

6.3 ABBILDUNGSVERZEICHNIS

6.4 TABELLENVERZEICHNIS

6.5 DANKSAGUNG

6.6 Eidesstattliche Versicherung 


\section{Einleitung}

\subsection{Betrachtung der Arbeitsprozesse im Krankenhaus}

Der Aspekt der Kosteneffizienz im Gesundheitswesen gewinnt unter dem ökonomischen Kostendruck besondere Relevanz für Ärzte, technische Assistenten und Pflegepersonal, insbesondere im Hinblick auf Qualität und Verfügbarkeit medizinischer Leistungen. Die Nachfrage nach medizinischer Leistungen ist in den letzten Jahren kontinuierlich angestiegen. Verantwortlich hierfür ist einerseits der technische Fortschritt sowie die Anwendung neuartiger Behandlungsmethoden und andererseits die epidemiologische Transition. Hierunter wird der steigende Anteil der älteren Menschen an der Bevölkerung sowie die damit einhergehende Zunahme chronischer Erkrankungen verstanden (67). Die dadurch bedingte steigende Nachfrage nach Gesundheitsleistungen bei gleichzeitiger Wachstumsschwäche der solidarischen Finanzierungsmöglichkeiten hat den Gesetzgeber dazu bewegt, neue Abbrechungssysteme einzuführen, um somit die medizinischen Leistungserbringer zu kosteneffektivem Handeln zu motivieren. So wurde 1995 zunächst auf freiwilliger Basis ein Fallpauschalensystem mit Sonderentgelten für bestimmte Leistungen eingeführt. Dessen langfristig angelegte Entwicklung konnte den stark steigenden Kostendruck im Gesundheitssystem nicht abfangen, so dass die Einführung der Vergütung auf Basis von Diagnosis Related Groups (DRG) zum Jahr 2003 beschlossen wurde. Mit dem DRG-System werden Kosten pauschal für Diagnosen vergütet. Die Höhe der Vergütung orientiert sich an in Studien ermittelten Kosten, die durchschnittlich bei einem Patienten mit der gegebenen Kombination von Diagnosen entstehen. Mit diesem Betrag muss der Leistungserbringer haushalten; es liegt jetzt in seiner Verantwortung, inn effizient zu nutzen. Resultierend wird eine erhöhte Motivation erwartet, die eigenen Prozesse unter dem Aspekt der Kosteneffizienz zu überprüfen und zu gestalten.

Betrachtet man die stationäre Behandlung eines Patienten im System Krankenhaus, lassen sich zwei Bereiche abgrenzen, die sich aus prozesstechnischer Sicht grundlegend unterscheiden. Im Versorgungsbereich verbringt der Patient den größten Teil seines Krankenhausaufenthalts. Hier finden die Pflege und Überwachung statt, die Informationen aus den einzelnen Diagnostik- und Behandlungssträngen laufen hier zusammen und dienen als Grundlage für weitere Entscheidungen. In hochspezialisierten Funktionsbereichen wie beispielsweise einer 
radiologischen Klinik werden einzelne diagnostische und therapeutische Maßnahmen durchgeführt. Während der Fokus im Versorgungsbereich auf dem Patienten liegt und sich die Abläufe nach seiner Symptomatik richten, sind die Abläufe innerhalb eines Funktionsbereichs in erster Linie durch die Maßnahmen determiniert, die hier durchgeführt werden. Im Rahmen eines Krankenhausaufenthalts durchläuft ein Patient zahlreiche dieser Funktionsbereiche. Diese Bereiche diagnostizieren oder therapieren eine Anzahl von Patienten in einem Routineprogramm. Für jeden einzelnen Patienten muss dabei eine hohe Ergebnisqualität erzielt werden. Das beinhaltet eine an der Fragestellung ausgerichtete und relevante diagnostische Aussage oder ein relevantes therapeutisches Ergebnis. Diese Vorgehensweise soll die Verfügbarkeit spezieller Einzelleistungen erhöhen, zu einer verbesserten Qualität der Einzelmaßnahme führen und ihre Kosten reduzieren. Der bestehende Kostendruck kann insbesondere durch eine effiziente Arbeitsablaufplanung bei radiologischen Dienstleistungen positiv beeinflusst werden (73). Auch für den Patienten ergeben sich bei einer verbesserten Prozesseffizienz Vorteile in Form von verringerten Warte- und Untersuchungszeiten, was die erlebte Prozessqualität steigert. Für den Funktionsbereich ergibt sich eine höhere Frequenz an Untersuchungen und ein geringerer Arbeitsaufwand, was niedrigere Kosten pro Untersuchung und eine raschere Amortisation von Investitionen erwarten lässt.

\subsection{Optimierung eines Arbeitsprozesses}

Das Leitmotiv des Prozessmanagements ist die Kundenorientierung, die als wesentliche Voraussetzung zur Erlangung von Wettbewerbsvorteilen gilt. Weitere Merkmale umfassen die Prozessorientierung als Kontrapunkt zur Betrachtung von Kliniken oder Bereichen, eine ganzheitliche Betrachtungsweise von Unternehmensprozessen unter Kosten-, Qualitäts- und Zeitaspekten sowie die langfristige Ausrichtung eines Verbesserungsprozesses

Kunden- oder Patientenorientierung wurde in der Medizin bislang in erster Linie mit möglichst guter medizinischer Versorgung nach aktuellem wissenschaftlichen Kenntnisstand gleichgesetzt. Der Begriff der Versorgung ist hier nicht zufällig gewählt. Er betont den Aspekt der Notwendigkeit der medizinischen Handlung: im Falle einer Erkrankung muss der Patient versorgt werden. Dabei ist das „Wie“ zweitrangig, solange die Behandlung medizinisch adäquat erfolgt. Das aus diesem 
Verständnis heraus entwickelte Gesundheitssystem regulierte sich über den Versorgungsbedarf. Die dafür notwendigen finanziellen Mittel waren lange Zeit im ausreichenden Maße vorhanden. Eine Konkurrenzsituation innerhalb des Systems entstand allenfalls regional über eine begrenzte Anzahl bestimmter Krankheitsbilder. Der zunehmende Kostendruck im Gesundheitswesen führte zu einer Neubewertung der Prioritäten des medizinischen Handelns. Neben der bisher angestrebten Effektmaximierung im Sinne einer medizinisch bestmöglichen Patientenbehandlung wachsen, forciert durch die Einführung der DRG, die Bestrebungen zur Effizienzsteigerung, d.h. Reduktion des Mitteleinsatzes für das angestrebte Behandlungsziel. Neben der Notwendigkeit, mit einem begrenzten Budget haushalten zu müssen, entsteht einerseits zunehmend die Motivation, aus dem festen Budget Gewinn zu erwirtschaften. Dies verändert andererseits die Haltung der Leistungserbringer gegenüber den Patienten. Das Selbstverständnis verschiebt sich vom Versorger zum Dienstleister, womit der Kunden- oder Patientenzufriedenheit vermehrt Aufmerksamkeit gewidmet wird. Kundenzufriedenheit bedeutet in diesem Zusammenhang: „...keine Abweichung zuzulassen von den identifizierten Kundenwünschen“

Die Prozessorientierung ist ebenfalls ein Ergebnis des Umdenkens zu mehr Kosteneffizienz. Sie entstand aus der Bestrebung, bei gleichbleibender oder verbesserter Ergebnisqualität, eine höhere Effizienz zu erzielen. Im Mittelpunkt der Betrachtung steht nicht mehr die behandelnde Klinik mit den dazugehörigen Funktionsbereichen, sondern der Behandlungsprozess. Er kann sich aus Leistungen verschiedener Bereiche zusammensetzen. Spätestens mit Einführung der DIN ISO 9000 erlangte die Prozessorientierung große Popularität (82). Mit Einführung der viel diskutierten Disease-Management-Programme sollen Einsparpotenziale durch prozessorientierte, eng verzahnte Zusammenarbeit unterschiedlicher medizinischer Disziplinen nutzbar gemacht werden. Die Ganzheitlichkeit im Prozessmanagement ist auch ein Anspruch des Total Quality Managements (TQM), einer Philosophie der andauernden Prozessverbesserung (17; 19; 32; 44). Sie umschreibt den Anspruch, Unternehmensprozesse unter Kosten-, Qualitäts- und Zeitaspekten zu steuern mit dem Ziel, alle vom Kunden wahrgenommenen Leistungsmerkmale zu berücksichtigen. Ihre Realisierung bedingt ein eng verzahntes Zusammenspiel verschiedener Instrumente und Methoden des Kosten- Qualitäts- und Time-Based-Managements sowie der Organisationslehre 
(82), was bei Betrachtung der bestehenden Verhältnisse im deutschen Gesundheitswesen eine umfangreiche Aufgabe ist. Je nach Richtung, aus der die Initiative zu diesem Ansatz kommt, wird ihr Schwerpunkt unterschiedlich gesetzt sein. Die langfristige Orientierung des Verbesserungseffekts entstand aus der Einsicht heraus, dass Rationalisierungsprojekte klassischen Musters (z.B. Business Reengineering, Gemeinkostenwertanalyse) meist nur einen kurzfristigen Effekt aufweisen. Sie sind dadurch charakterisiert, dass eine Verbesserung durch eine singuläre Intervention erreicht werden soll. Neuere Ansätze des Reengineerings zielen auf einen kontinuierlichen Verbesserungsprozess in kleinen Schritten ab. Dazu werden in einem Reengineeringvorhaben Prozessdenken, Prozessorganisation und Prozessmanagement etabliert, durch die ein langfristig wirksamer Verbesserungskreislauf eingeleitet wird (82). Zahlreiche Werkzeuge wurden entwickelt, um Prozesse darzustellen und umzustrukturieren. Im englischen Sprachgebrauch wird die Summe all jener Maßnahmen in langfristiger Anwendung unter dem Begriff Continuous Quality Improvement (CQI) zusammengefasst (z.B. (3)).

Die Optimierung eines Arbeitsprozesses (im Folgenden synonym zum Begriff Arbeitsablauf verwendet) ist ihrerseits ein kontinuierlicher Prozess. Es existieren zahlreiche Methoden, die im Rahmen des Prozessmanagements zur Analyse und Optimierung von Prozessen eingesetzt werden. Sie kommen auf verschiedenen Ebenen zum Einsatz, die einerseits weiche Faktoren wie z.B. Konfliktmanagement sowie kommunikations- und motivationsfördernde Maßnahmen und andererseits harte Faktoren wie die Planung, Steuerung und Kontrolle aller formalen Aspekte eines Prozesses betreffen. Zu den formalen Aspekten zählen z.B. die personelle und die Geräteausstattung sowie die Struktur des Arbeitsprozesses oder des Informationsflusses. Welche Maßnahme des Prozessmanagements wann eingesetzt wird, richtet sich nach der Art des Prozesses und dem Optimierungsziel. Generell wird man eine Methode wählen, die mit geringst möglichem Aufwand eine effektive Verbesserung des Prozesses im Hinblick auf das wahrgenommene Problem ermöglicht. 


\subsection{Methodik des Prozessmanagements}

Zu Beginn einer jeden Prozessoptimierung steht das subjektiv empfundene Problem (z.B. Beschwerden von Patienten über lange Wartezeiten). Der erste Schritt im Prozessmanagement ist es, dieses Problem genau zu formulieren (Die Wartezeiten sind zu lang). Daraus ergibt sich eine umschriebene Aufgabenstellung für ein Optimierungsprojekt (Die Wartezeiten müssen verkürzt werden). Aus der Aufgabenstellung lassen sich Fragestellungen ableiten:

1. Wie lang sind die Wartezeiten wirklich?

2. Wo sind die Ursachen für lange Wartezeiten?

3. Welche Maßnahmen können die Wartezeiten verkürzen?

Erst danach erfolgt die Auswahl der passenden Methode zur Prozessoptimierung. Eine Prozessoptimierung betrifft immer ein Gruppe von Prozessbeteiligten. Jedes Gruppenmitglied bringt seine eigene Perspektive in den Prozess mit ein. Entscheidend für das Gelingen einer Prozessoptimierung ist die Einigkeit aller Gruppenmitglieder sowohl über das Problem als auch über die Maßnahmen zur Optimierung. Man sollte sich nicht zu schade sein, dass Problem in der Gruppe zu formulieren und schriftlich zu fixieren. Auch die Auswahl der Methodik sollte im Konsens erfolgen. Voraussetzung für eine Prozessoptimierung ist die Abbildung des bestehenden Prozesses in einem Modell. Innerhalb einer Gruppe aus mehreren Personen bedarf es dazu formaler Methoden. Die einfachsten und auch am häufigsten eingesetzten Verfahren werden unter den Begriff "Common Sense Methoden“ zusammengefasst. Viele Probleme lassen sich durch Beobachtung und logisches Überlegen Iösen. Hierzu werden keine komplexen Prozessmanagementmethoden benötigt. Als Hilfestellung können einfache papier- oder computerbasierte Darstellungsformen eingesetzt werden (z.B. Organigramme, Fish bone-Diagramme). Viele Arbeitsvorgänge, an denen mehrere Personen beteiligt sind (z.B. Anfertigung einer Computertomographie unter Beteiligung von MTRAs und Ärzten), sind aufgrund der Interaktionen und Abhängigkeiten wesentlich komplexer als sie zunächst erscheinen. Die simplen z.B. papierbasierten Methoden sind bei höheren Komplexitätsgrad überfordert, d.h. sie liefern nicht die nötige oder zutreffende Aussage, um das Problem zu lösen. Abbildung 1-1 zeigt den Zusammenhang von Komplexität, Aufwand und Nutzen für den Anwender. 


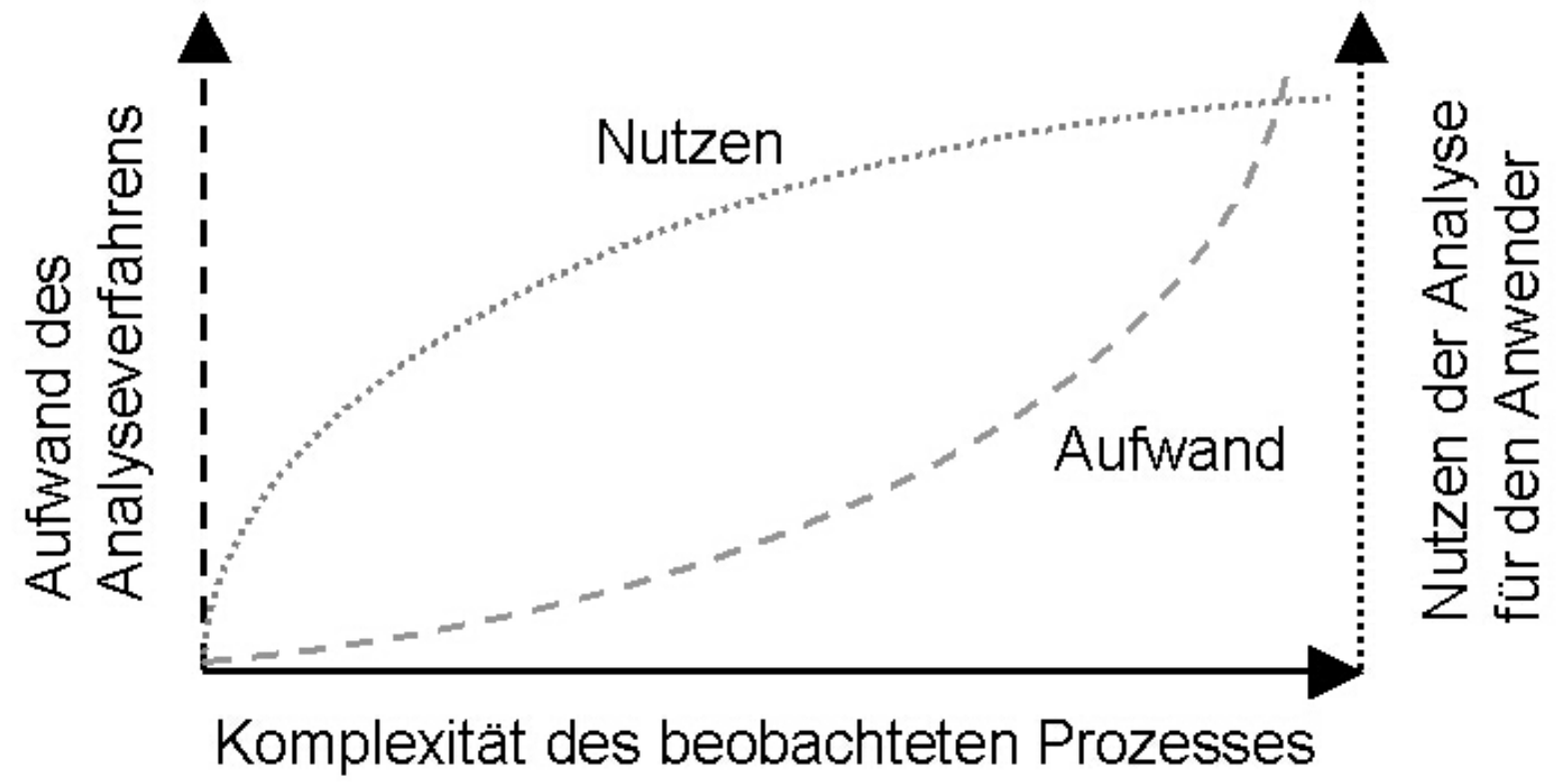

Abbildung 1-1: Zusammenhang von Komplexität, Aufwand und Nutzen für den Anwender bei Anwendung von Prozessmanagementmethoden.

\subsubsection{Netzplantechnik}

In den vergangenen Jahrzehnten wurden Strategien in amerikanischen Krankenhäusern entwickelt, um den Verbrauch an Ressourcen zu verringern sowie gleichzeitig den Qualitätsstandard zu halten oder zu verbessern $(31 ; 98 ; 102 ; 105$; 106). Dabei zeigte sich vor allem die Netzplantechnik des kritischen Weges - Critical Pathway Method (CPM) - als besonders erfolgreich, diesen Ansprüchen gerecht zu werden (46; 103). Der kritische Weg stellt die ideale Ablauffolge von Arbeitsschritten dar, um ein geplantes Ergebnis in möglichst kurzer Zeit zu erlangen. Netzplantechnik ist der zentrale Begriff der DIN 69900 Historisch gehen diese Ansätze der Netzplantechnik auf drei unabhängig voneinander entwickelte Verfahren zurück:

\subsubsection{Metra-Potential-Methode (MPM):}

Dieses Verfahren wurde 1956 in den USA entwickelt und verwendet Vorgangspfeilnetze. Ursprünglich wurde diese Methode in den Bereichen von Bauprojekten und technischen Entwicklungsprojekten angewendet, in denen sie nunmehr seit Jahrzehnten erfolgreich zum Einsatz kommen. Heute ist CPM noch vielfach ein Synonym für Vorgangspfeilnetze. Man spricht auch von CPMNetzplänen. 


\subsubsection{Program Evaluation and Review Technique (PERT):}

Dieses Verfahren, bei dem ursprünglich nur Ereignisknotennetze verwendet wurden, wurde ebenfalls 1956 in den USA entwickelt, und zwar speziell für die Planung und Überwachung militärischer Forschungs- und Entwicklungsprojekte. Es hat in seiner ursprünglichen Form kaum noch Bedeutung, wird aber manchmal als Synonym für Netzplantechnik verwendet.

Aus diesen fast zeitgleich entstandenen Ansätzen haben sich eine Vielzahl weiterer Netzplantechniken für spezielle Anwendungen in Industrie und Militär entwickelt (z.B. Precedence Diagramming Method (PDM), Graphical Evaluation and Review Technique (GERT)). Mittlerweile findet man für diese Verfahren hochentwickelte Anwendungsprogramme, die Anfangs- und Endzeitpunkte, den kritischen Weg, Pufferzeiten und andere nützliche Daten für große Projekte ermitteln. Erweitert erfolgen auch Berechnungen für optimistische, erwartete und pessimistische Zeitschätzungen, die der entsprechen. Im Gesundheitswesen wurden Überlegungen zur Anwendung dieser Verfahren in klinischen Abläufen erstmals in den frühen 70igern angestellt (9; 38; 69). 1974 stellte Shoemaker fest, dass Routine- oder Patientenprotokolle sinnvoll eingesetzt werden könnten, um eine umfassende Patientenversorgung und eine Evaluation des Heilungsfortschritts und des therapeutischen Nutzens zu gewährleisten. (86) Obwohl diese Vorschläge in Ansätzen Erfolge vorweisen konnten, fehlte zur damaligen Zeit der finanzielle Druck auf die Krankenhäuser, die ihre Kosten noch 1:1 bei den Versicherungen in Rechnung stellen konnten. Seitens der Ärzte wurde daher jegliche Einschränkung ihrer Behandlungsmethoden abgelehnt. Mit Beginn der 80iger Jahre rückten im Rahmen der Einführung prospektiver Haushaltführung der Krankenhäuser diese Ansätze wieder in den Vordergrund (21; 77). Begleitend machten die zum Teil enormen Prozessvarianten in den Behandlungsmethoden(es existierten große Variationen in der Behandlung eines Krankheitsbildes) die Problematik der Ineffizienz bewusst (26; 99). Dies führte dazu, dass immer mehr Kliniken in den Vereinigten Staaten vor allem im Pflegebereich (28) Programme (40) zur Umsetzung von Netzplanverfahren einführten. Eine Schlüsselmotivation für die Entwicklung von kritischen Wegen war die Verringerung der Liegedauer im Krankenhaus und damit der Kosten, die mit besonderen Diagnosestellungen und Behandlungsmethoden zusammenhängen (73). Auch in angrenzenden Disziplinen wie der Pharmazie (49) und der Forschung (51) wurde die 
Netzplantechnik erfolgreich eingesetzt. Weitere Vorteile sind in der Tabelle 1-1 angegeben.

Tabelle 1-1: Auswirkungen und Vorteile durch die Anwendung von Netzplantechnik (CPM/PERT)

\begin{tabular}{|lr|ll|}
\hline Förderung & Reduktion & \\
\hline Die Qualität der Pflege wird gesteigert & $\begin{array}{l}\text { Verringerung von Verspätungen und } \\
\text { Vebrauch von Ressourcen }\end{array}$ \\
\hline $\begin{array}{l}\text { Erleichterung, angestrebte Ziele zu } \\
\text { erreichen }\end{array}$ & Redutkion von Prozessvarianten \\
\hline $\begin{array}{l}\text { Verbesserung des } \\
\text { Leistungsverhältnisses }\end{array}$ & Preis- & $\begin{array}{l}\text { Verringerung der Liegedauer im } \\
\text { Krankenhaus }\end{array}$ \\
\hline
\end{tabular}

Die Entwicklung in den 90igern war durch die Berücksichtigung aller Behandlungsaspekte und individuellen Nutzungsmöglichkeiten der Netzplantechnik im klinischen Alltag geprägt (13; $18 ; \quad 50 ; \quad 51 ; \quad 55)$. Auch im Bereich der Radiologie wurde die Notwendigkeit von kosten- und ressourcensenkenden Maßnahmen erkannt (36; 74). Erfolge nach Änderungen im Arbeitsablauf im Umfeld von computertomographischen Untersuchungen wurden 1994 von Rhea et al. veröffentlich (75). Danach konnten nach dem Einsatz von Prozessmanagementtechniken die Kosten um 25\% verringert und der Patientendurchlauf um 50\% erhöht werden. Dies wurde durch den intensivierten Arbeitseinsatz während der Durchführung und der Reduzierung von ungenutzter Zeit erreicht. Dabei wurden zeitintensive und variable Vorgänge im Bezug auf den Patientendurchlauf kritisch durchleuchtet und ausgewertet. Anschließend erfolgte die Umsetzung in einem Netzplan mit begleitenden Infrastrukturumbauten wie den Einsatz von PACS und Mitarbeitereinweisungen. Die Ergebnisse zeigen dabei eine Verkürzung der Untersuchungsdauer von durchschnittlich 50 auf 35 Minuten auf. Die Verwendung der Netzplantechnik bei CT-Untersuchungen konnte in einer Studie erstmals die CPM/PERT-Methode mit der Berechnung von optimistischen, realistischen und pessimistischen Arbeitsabläufen gezeigt werden (94). Auf dem Gebiet der klinischen Radiologie wurde zuletzt im Massachusetts General Hospital in Boston, USA die Netzplantechnik zur erweiterten Zeitplanung und Qualitätskontrolle eingeführt. Hintergrund dieser Erhebung ist der zunehmende Konkurrenzdruck durch 
kleinere Institute und damit die zunehmend wichtigere Rolle der Zufriedenheit des Patienten (22). Parallel setzte die steigende Zahl der Institute, die ihre Radiologie durch den Einsatz von PACS filmlos machten, eine strukturierte Planung zur Effizienzsteigerung voraus

Zur Erstellung eines Netzplanes sollte eine Strukturplanung in vier Phasen erfolgen. Eine radiologische Untersuchung (z.B. Computertomographie) entspricht dabei dem durchzuführenden Projekt. Die Ereignisse entsprechen den Arbeitsschritten (=Vorgängen) der Untersuchung:

Tabelle 1-2: Erstellung eines Netzplans in vier Phasen

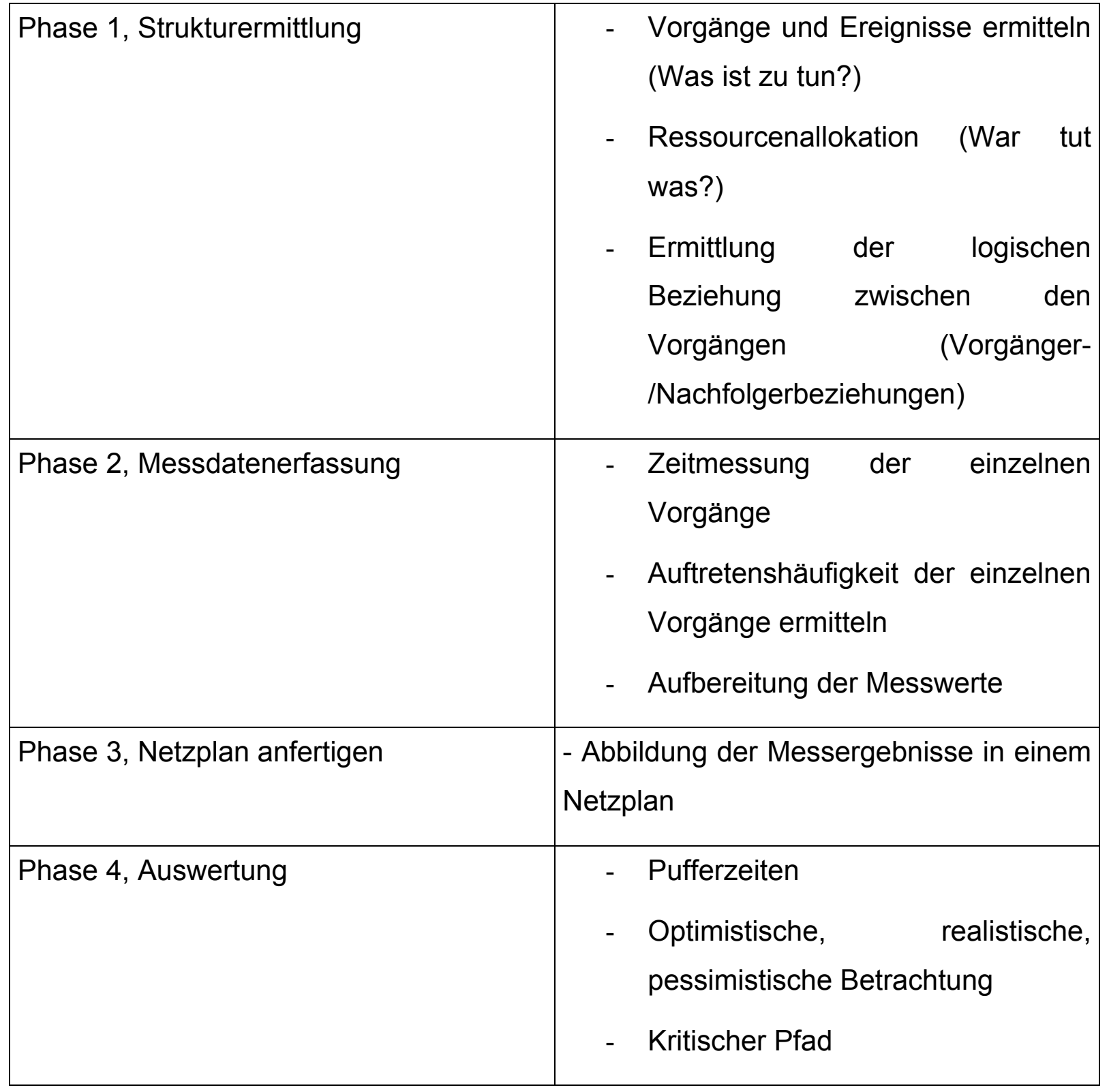

Durch die Zeitmessungen erfolgt eine Bestimmung der Vorgangs- und Verknüpfungsdauern der Arbeitsschritte, bzw. der Wahrscheinlichkeitsverteilung 
dieser Zeiten. Die Anordnung von Arbeitsschritten erfolgt im Netzplan in einer deterministischen zeitlichen Reihenfolge. Abbildung 1-2 zeigt ein Beispiel eines Netzplans einer computertomographischen Untersuchung. Auf der Grundlage eines Netzplanes können Gesamtprojektdauern (=Gesamtuntersuchungszeiten) und die Zeitreserven bestimmt werden. Unter Zeitreserven werden dabei vor allem die Pufferzeiten verstanden. Die Pufferzeit gibt den Zeitraum an, um welchen ein Arbeitsschritt verlängert werden kann, ohne dass sie Gesamtuntersuchungsdauer überschritten wird. Arbeitsschritte, die keine Pufferzeiten besitzen sind kritische Vorgänge, d.h. diese Arbeitsschritte besitzen keine Zeitreserven. Auf der Basis der gemittelten Zeitwerte können differenzierte Netzpläne mit drei unterschiedlichen Zeitschätzungen berechnet werden (siehe folgende Liste).

1. Optimistische Zeit: geringste Zeitdauer aufgrund eines verzögerungsfreien Untersuchungsablaufs (optimales Untersuchungstiming).

2. Pessimistische Zeit: extrem erhöhte Zeitdauer als Folge aller denkbaren Verzögerungen bei der Untersuchung.

3. Realistische Zeit: die Bestimmung erfolgt durch eine gewichtete Berechnung der gemittelten Zeitwerte aus der Zeitmessung mit optimistischen und pessimistischen Zeitberechnungen nach einer Formel (s.u.)

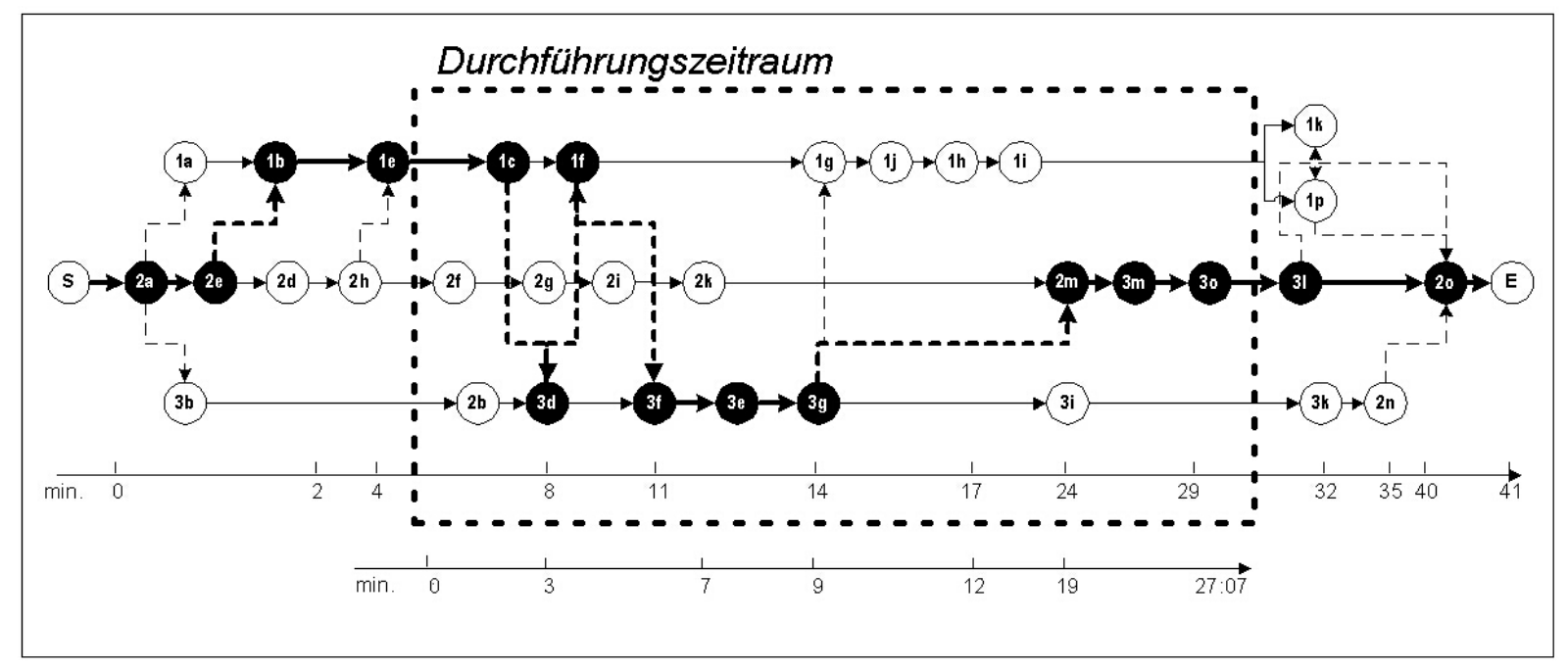

Abbildung 1-2: CPM/ PERT Netzplan einer CT-Untersuchung unter optimistischen Bedingungen. Kritischer Weg in schwarz markiert.

Bei der Netzplantechnik werden als wesentliche Einflussgrößen Zeit, Kosten und Ressourcen berücksichtigt. Die Verfügbarkeit leistungsfähiger Software macht es mittlerweile aber auch für kleinere und dynamische Projekte möglich, Netzplantechnik sinnvoll einzusetzen. Eine wesentliche Einschränkung der 
Netzplantechnik ist, dass Projekte einzigartig in ihrer Konfiguration nur einmal stattfinden. Es wird ein Untersuchungsablauf nur isoliert abgebildet. Im klinischen Alltag finden Überschneidungen bei der Durchführung von diagnostische Untersuchungen statt, d.h. während beispielsweise eine Computertomographie durchgeführt wird, wird bereits der nächste Patient vom Arzt aufgeklärt oder der Befund vom vorherigen Patienten geschrieben. Für die Prozessplanung und evaluation hat dies verschiedene Konsequenzen. Ein Netzplan kann nur ein statisches Abbild einer speziellen Prozesskonfiguration darstellen. Einschränkungen entstehen dadurch beispielsweise in der Beurteilung der Multikolinearität (56) sowie der Wechselwirkung verschiedener Objekte untereinander, mit Ressourcen und mit der Prozessstruktur. Prozessvariablen, die sich im Verlauf des Prozesses verändern und damit wiederum den Prozessablauf beeinflussen, lassen sich nicht darstellen. Die Dynamik eines Arbeitsprozesses lässt sich erst in der Simulation beurteilen. Der Einfluss z.B. sporadisch auftretender Ereignisse auf den durchschnittlichen Untersuchungsaufwand und die durchschnittliche Untersuchungsdauer ist erst mit einer großen Anzahl von Prozessdurchläufen unter verschiedenen Voraussetzungen zutreffend zu beurteilen. So wird im Simulationsmodell mit stochastisch modellierten Vorgangsdauern und Objektzahlen gearbeitet, um Wechselwirkungen zwischen den Ressourcen, Vorgängen und Objekten auch unter Extrembedingungen zu erkennen.

\subsubsection{Prozesssimulation}

Die Prozesssimulation wurde in den letzten zwei Jahrzehnten entwickelt, um Prozesse der Fertigungstechnik zu untersuchen. In den 90er Jahren fand sie Eingang in das Unternehmensmanagement, wo sie die Transparenz und den Ablauf aller Arten von Prozessen (Fertigungs-, Geschäfts-, Arbeitsprozesse) verbesserte. Prozesssimulation ist ein Werkzeug, mit dem die Dynamik von Prozessabläufen in Systemen visualisiert und evaluiert wird. Der Einfluss z.B. sporadisch auftretender Vorgänge auf den durchschnittlichen Untersuchungsaufwand und die durchschnittliche Untersuchungsdauer kann anhand einer großen Anzahl von Prozessdurchläufen unter verschiedenen Voraussetzungen zutreffend zu beurteilen Dabei handelt es sich um eine Simulation, also einer Imitation der Realität oder eines als Konzept existierenden Prozesses bzw. Systems in einem bestimmten Zeitraum. Die Prozesssimulation wird verwendet, um das Verhalten eines Systems zu beschreiben und zu analysieren, „was wäre wenn“-Fragen über das reale System zu 
beantworten und um bei der Planung eines realen Systems zu helfen (8). So wird im Simulationsmodell mit stochastisch modellierten Vorgangsdauern und Objektzahlen gearbeitet, um Wechselwirkungen zwischen den Ressourcen, Vorgängen und Objekten auch unter Extrembedingungen zu erkennen. In vielen Fällen beginnt ein neuer Prozesszyklus bereits, bevor der aktuelle abgeschlossen ist. Bei radiologischen Untersuchungen kommen Patienten bereits an und werden registriert, während vielleicht ein anderer Patient noch untersucht und für einen dritten gerade der Befund geschrieben wird. Dabei laufen drei Untersuchungen gleichzeitig ab. Damit hierbei die Überlappungseffekte und ihr Einfluss auf den Ressourcenbedarf korrekt widergegeben werden, geriete ein statischer Netzplan sehr komplex. Komplexe Interaktionen entstehen auch durch die asymmetrische Konfiguration von Untersuchungsplätzen und administrativer Instanz. Eine administrative Instanz arbeitet häufig für mehrer Arbeitsplätze gleichzeitig. Sie muss ihre Arbeitskraft je nach Bedarf auf mehrere Einheiten verteilen. Diese haben variierende Zykluszeiten je nach Untersucher und auch anderen Faktoren. Soll hier der Einfluss wechselnder personeller Besetzung in Administration und klinischer Tätigkeit untersucht werden, müssen die verschachtelten, zeitversetzt ablaufenden Prozesszyklen mit ihren wechselseitigen Einflüssen nachgebildet werden. Mit der statischen Netzplantechnik ist dies nicht zu verwirklichen. Prozesssimulation ist ein relativ aufwändiges Verfahren. Der Aufwand für die Datensammlung bewegt sich zwar im üblichen Rahmen einer wissenschaftlichen Studie. Jedoch erfordert die Modellerstellung mit Testen, Verifizierung, Validierung und Evaluierung der verschiedenen Szenarien einen erheblichen zusätzlichen personellen Aufwand, der über eine rein statistische Auswertung weit hinaus geht. Der Vorteil zuverlässiger, prospektiver Aussagen muss in Relation zu diesem Aufwand gesetzt werden. 


\section{Zielsetzung}

Durch die Einführung der G-DRG (German - Diagnosis Related Groups) wird die Verantwortung für wirtschaftliches Arbeiten in den Krankenhäusern auf den Leistungserbringer übertragen. In einem Verbund von Fachkliniken beeinflusst der Radiologe wesentlich die Liegezeiten von Patienten durch die diagnostische Information und die Verfügbarkeit seiner Dienstleistungen. Die Organisation und zeitliche Bemessung von radiologischen Untersuchungsmethoden wird dadurch um so wichtiger. Der Arzt wird im Rahmen seines Studiums und seiner Facharztweiterbildung nicht im Organisationsmanagement ausgebildet. Trotzdem wird vom Arzt über die medizinische Versorgung hinaus zunehmend verlangt, dass er seine Arbeitsabläufe auch unter wirtschaftlichen Gesichtspunkten optimiert. In der vorliegenden Arbeit werden Methoden des Prozessmanagements für ihre Anwendung in der Radiologie anhand von eigenen wissenschaftlichen Arbeiten vorgestellt:

1. Es werden die Arbeitsabläufe beim Einsatz der mobilen Computertomographie auf der Intensivstation mit der ortsfesten CT in der radiologischen Klinik verglichen.

2. Anwendung der Netzplantechnik zur Bestimmung der minimalen Untersuchungszeiten in der klinischen Routine bei abdominellen Ultraschalluntersuchungen.

3. Anwendung der CPM / PERT Methode an einem Ultraschallarbeitsplatz in einer radiologischen Klinik.

4. Anwendung der Netzplantechnik für computertomographische Untersuchungen in der diagnostischen Radiologie und Bestimmung von optimistischen, realistischen und pessimistischen klinischen Wegen (critical pathway).

5. Einsatz der Prozesssimulation zur quantitativen Abschätzung der Effizienzsteigerung an einem Routineultraschallarbeitsplatz durch eine digitale Infrastruktur. 


\section{Arbeitsprozessanalyse für den Einsatz der mobilen \\ 3.1 Computertomographie auf der Intensivstation}

3.1.1

Einleitung

\subsubsection{Mobile Diagnostik}

Die diagnostische Radiologie verwendet sehr verschiedene Instrumente, welche sowohl für die stationäre Anwendung in der radiologischen Klinik als auch für den Einsatz am Patientenbett verfügbar sind. Auf der Intensivstation werden häufig konventionelle Röntgenaufnahmen benötigt, aber auch im Operationssaal, beispielsweise, wenn komplizierte Frakturen reponiert werden müssen. Für diese Aufgaben stehen fahrbare Röntgengeräte zur Verfügung, die ohne großen technischen Aufwand mobil zu betreiben sind. Die interventionelle Durchleuchtung wird ebenfalls je nach Indikation stationär oder mobil durchgeführt. Beispielsweise leistet die mobile digitale Subtraktionsangiographie (DSA) im OP gute Dienste bei gefäßchirurgischen Eingriffen (10). Für die Radioisotopen gestützte Diagnostik außerhalb des nuklearmedizinischen Funktionsbereichs stehen mobile GammaKameras zur Verfügung. Bei Verdacht auf Lungenembolie können damit Szintigramme des pulmonalen Gefäßsystems von instabilen Patienten auf der Intensivstation erstellt werden (48). Sonographiegeräte werden aufgrund der kompakten Bauweise seit ihrer klinischen Einführung mobil verwendet. Im Gegensatz dazu wurden Computertomographen wegen der zugrundeliegenden technischen Prinzipien und der baulichen Komplexität bis vor kurzem nur als stationäre Geräte konzipiert. Jedoch gibt es seit neuerem mobil einsetzbare Computertomographen wie den Tomoscan M (Philips, Utrecht, Niederlande), wodurch CT-Untersuchungen vor Ort auf der Station oder im OP möglich geworden sind. Es sind vielfältige Einsatzmöglichkeiten für die mobile Computertomographie denkbar (23; 43; 52; 58; 59; 90; 92). In unserer Institution kommt die mobile CT in der Notaufnahme, in der Angiographie, im Operationssaal und auf den Intensivstationen zum Einsatz. Durch die flexiblen Einsatzmöglichkeiten aufgrund der Zulassung in verschiedenen Kliniken unseres Hauses wird dem mobilen Konzept des Geräts Rechnung getragen. Insbesondere wird die mobile CT in der Notaufnahme und auf den Intensivstationen durchgeführt. Gerade in diesen Bereichen könnte die mobile CT bei kritischen Patienten, für die ein Transport in die radiologische Klinik ein schwer kalkulierbares Risiko darstellt, eine Verbesserung der Intensivüberwachung und erzielen 
In der vorliegenden Studie werden die Arbeitsabläufe für den Einsatz der mobilen CT unmittelbar auf der Intensivstation im Vergleich zur ortsfesten CT in der radiologischen Klinik verglichen. Im weiteren werden die Arbeitabläufe der mobilen CT und dessen Akzeptanz beim intensivmedizinischen Personal im Vergleich zur CT in der radiologischen Klinik evaluiert.

\subsubsection{Material und Methodik:}

Für diese Studie wurde der mobile Computertomograph Tomoscan M der Firma Philips (Utrecht) verwendet. Das Gerät besteht aus den drei Komponenten Gantry, Untersuchungstisch und Steuerkonsole, von denen jede auf vier Rollen für den mobilen Einsatz gelagert ist (Abbildung 3-1 bis 3-3)

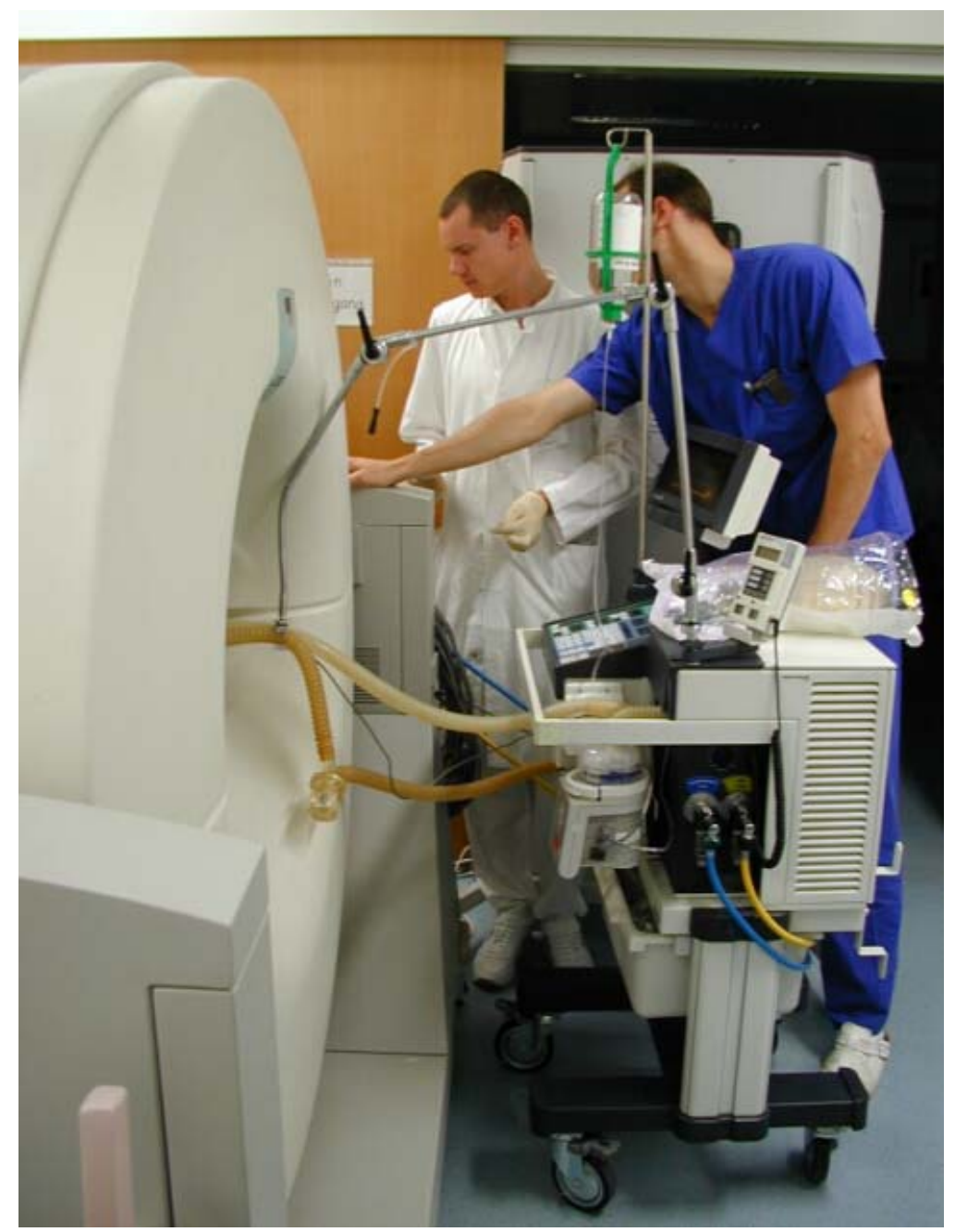

Abbildung 3-1: Gantry mit Beatmungseinheit 


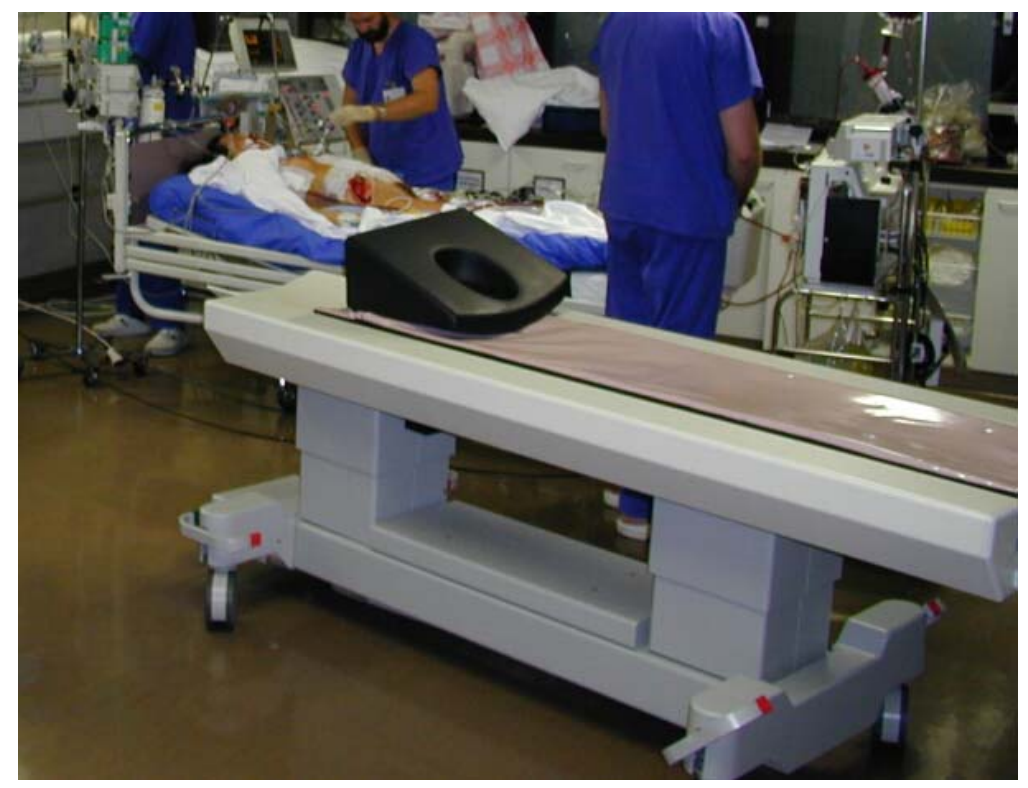

Abbildung 3-2 CT-Untersuchungtisch

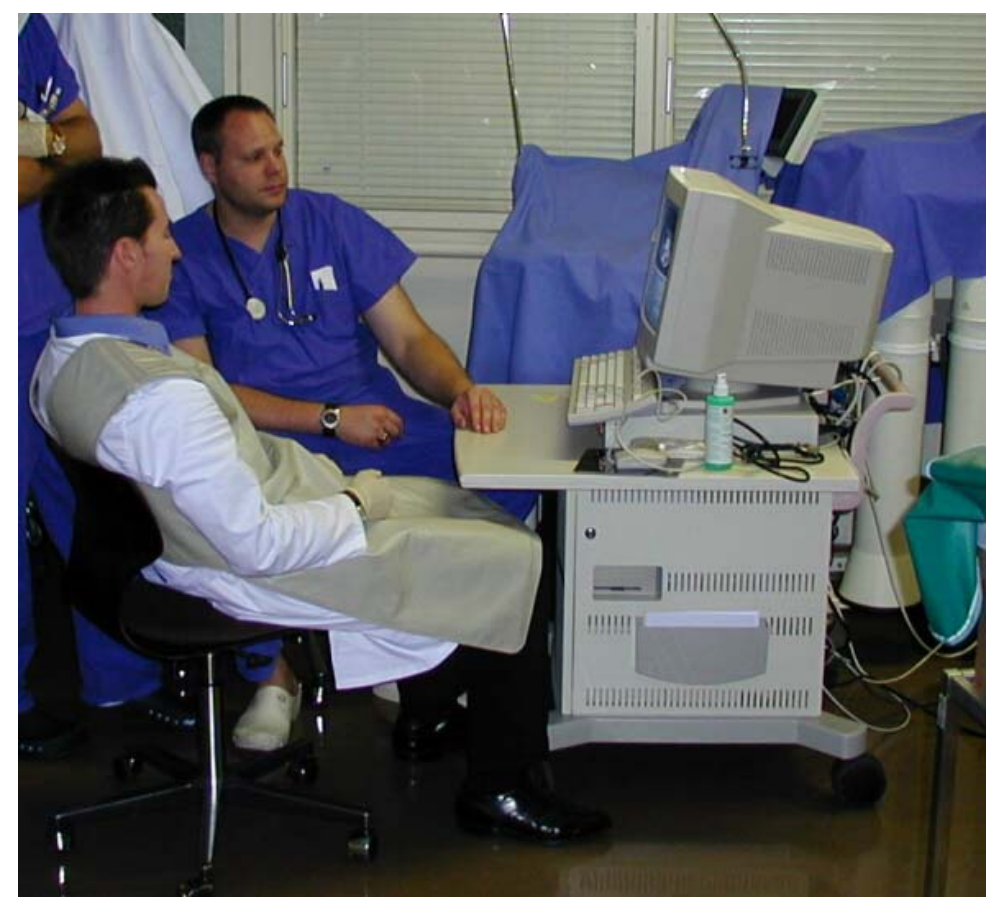

Abbildung 3-3: CT-Computerkonsole (Steuereinheit)

Für die Anfertigung einer CT-Untersuchung sind nur die Gantry und die Steuerkonsole unverzichtbar. Aufgrund der Möglichkeit von Translationsbewegungen der Gantry lassen sich kranielle CT-Untersuchungen auch ohne Verwendung des CT-Tisches durchführen, wenn der Patient in seinem Bett liegt (42). Die modulare Bauweise des Tomoscan M ist eine wesentliche Grundlage seiner Mobilität. Die einzelnen Komponenten können schnell und unkompliziert über Steckverbindungen voneinander getrennt und separat transportiert werden. Für den Transport der 
Komponenten bedarf es auf ebenen Strecken jeweils nur einer Person. Besonders bezüglich der Gantry, die mit $460 \mathrm{~kg}$ die schwerste Komponente darstellt, ist es jedoch wesentlich praktikabler, wenn mindestens zwei Personen für den Transport des Geräts zur Verfügung stehen. Die Gantry passt wie ein Patientenbett mit ausreichend Abstand zu den Wänden in die Fahrstühle des Klinikums, was eine wesentliche Voraussetzung für ihre Mobilität darstellt. Die Computerkonsole und die Gantry verfügen jeweils über eigene Netzstecker für die externe Stromversorgung. Bei vollem Ladezustand der Akkumulatoren können mehrere CT-Untersuchungen unabhängig vom Stromnetz durchgeführt werden. Der Durchmesser der Untersuchungsöffnung beträgt nur $60 \mathrm{~cm}$, im Gegensatz zu einer herkömmlichen CT Gantryöffnung von $70 \mathrm{~cm}$.

\subsubsection{Einsatzorte}

Die mobile CT kommt in Abhängigkeit vom Bedarf an unterschiedlichen Einsatzorten im Klinikum zum Einsatz. In der vorliegenden Studie wurde die Evaluierung der mobilen CT auf einer internistischen Intensivstation vorgenommen. Der Großteil der Untersuchungen erfolgte auf der Intensivstation in einem speziellen Interventionsraum der Station. Der Raum ist ein zentrales Element der Station und von den Patientenzimmern aus leicht zu erreichen. Im Interventionsraum stehen neben der mobilen CT verschiedene diagnostische Verfahren wie Durchleuchtung, Ultraschall, Endoskopie zur Verfügung. Zusätzlich sind OP-Leuchten und ein Intensivmonitor an der Decke befestigt (Abbildung 3-4).

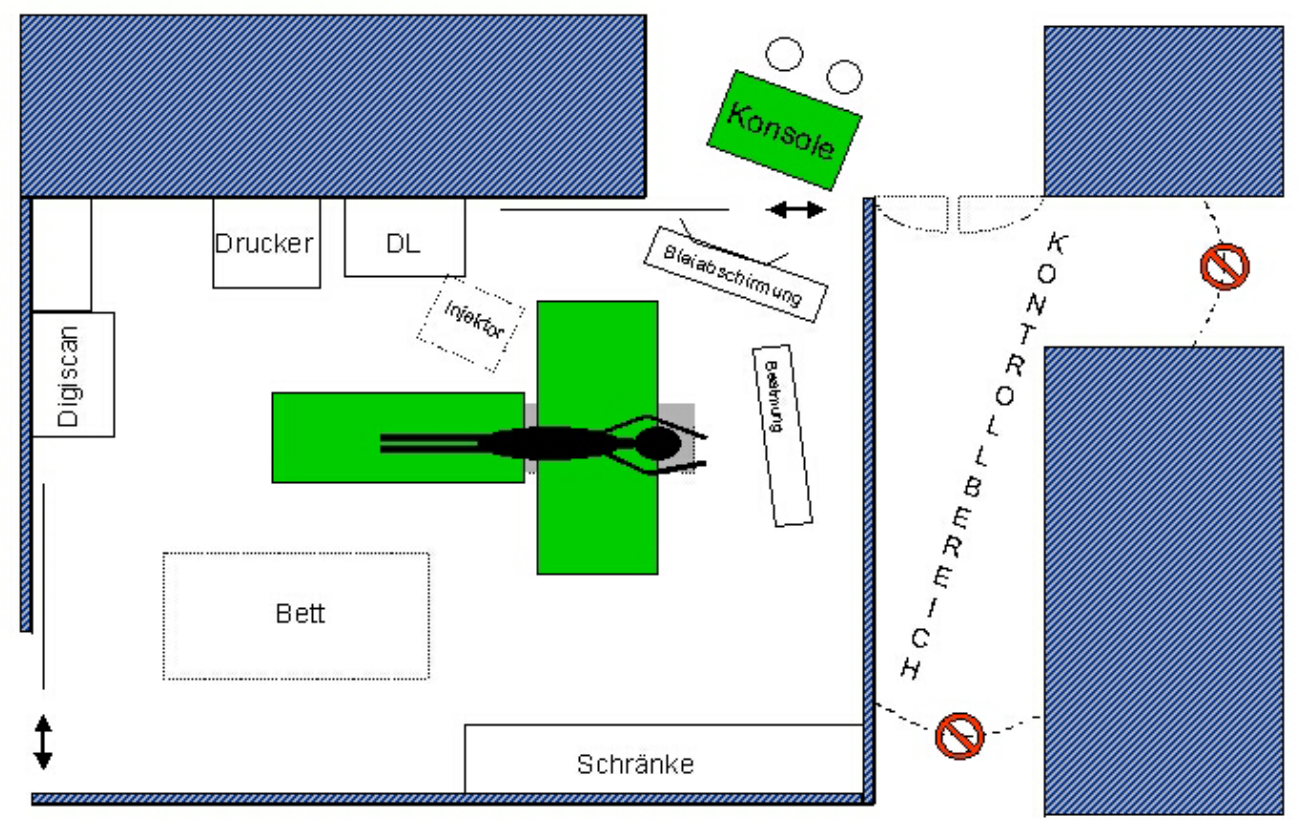

Abbildung 3-4: Grundriss des Interventionsraums auf der Intensivstation 
Darüber hinaus bestand auch die Möglichkeit der CT-Untersuchung direkt in bestimmten Patientenzimmern. Der relativ hohe bauliche Aufwand zum Strahlenschutz war der Grund für die Nutzung des Interventionsraums als Hauptuntersuchungsort.

Es wurden insgesamt 44 konsekutive CT-Untersuchungen mit der Stoppuhr begleitet. Je 22 intensivpflichtige Patienten wurden entweder mit der mobilen CT oder in der radiologischen Klinik untersucht. Die ermittelten Zeitwerte der einzelnen CTArbeitsschritte wurden für die statistische Analyse in einer Rangfolge-Skala angeordnet. Die CT-Untersuchung wurde für die Zeitmessung in einzelne Arbeitsschritte untergliedert: Vorbereitung, Hintransport, Umlagern, Untersuchung, Umlagern, Rücktransport, Nachbereitung. Die Vorbereitung (Vorgang 1) des Patienten für Transport und Untersuchung stellte den ersten Arbeitsschritt dar. Zur Vorbereitung gehörte die Diskonnektion des Patienten von Perfusoren und Infusomaten mit nicht lebensnotwendigen Medikamenten. Des weiteren beinhaltete sie die Umstellung von der stationären auf eine mobile Beatmungseinheit (Oxylog Fa. Drager, Medumat) sowie gegebenenfalls eine Intensivierung der Sedierung. Die Zeitmessung begann, sobald der erste Handgriff für die Vorbereitung von Arzt oder Pflegekraft getätigt wurde. Wenn der Patient bereit für den Transport war, endete die Erfassung der Vorbereitungszeit. Die Transportzeit (Vorgang 2) begann, sobald das Bett vom Intensivplatz entfernt werden konnte und endete bei Ankunft am CT-Untersuchungsplatz, d.h., dass sich auch Wartezeiten auf den Transportdienst oder ein noch nicht einsatzbereites CT zur Transportzeit

hinzuaddierten. Nach der Ankunft des Patienten im Untersuchungsraum erfolgte seine Umlagerung (Vorgang 3) auf die CT-Liege. Der Untersuchungsvorgang (Vorgang 4) war in zwei Teilschritte gegliedert. Der erste Teilschritt beinhaltete die Positionierung der CT-Liege in eine für die Untersuchung geeignete Stellung. Der zweite Teilschritt bestand in der Bildakquisition. Bei Einsatz von Kontrastmittel wurden die Zeiten zur Einrichtung der Kontrastmittelpumpe sowie die Zeiten von Nativ- und Kontrastmittelaufnahmen addiert. Der Untersuchungsvorgang endete, sobald die letzte Aufnahme erfolgt war. In unserer Studie wird nicht in Nativ- und Kontrastmitteluntersuchung unterschieden, da die Anteile beider Verfahren an der Gesamtzahl der Untersuchungen mit mobiler und 
stationärer

CT

gleich

sind.

Nach Beendigung der Untersuchung laufen die Arbeitsschritte 1-3 in umgekehrter

Reihenfolge ab: Umlagerung (Vorgang 5), (Rück-) Transport (Vorgang 6), Nachbreitung (Wiedereinbindung des Patienten in seinen Intensivplatz (Vorgang 7). Die Zeiten von Hin- und Rücktransport wurden für die Gesamttransportzeit addiert. Entfernungsbestimmung mit Distanzlaufrad: auf der nephrologischen Intensivstation wurden die Entfernungen der Patientenzimmer zur mobilen CT im Interventionsraum bestimmt. Weiterhin wurden die Distanzen der Patientenzimmer aller Intensivstationen zu den CT-Untersuchungsräumen in der radiologischen Klinik erfasst. Evaluierung des Intensivpersonals mittels anonym auszufüllendem Fragebogen: Es wurde ein Fragebogen erstellt, der die subjektive Einschätzung des Intensivpersonals der nephrologischen Intensivstation anhand folgender Parameter evaluiert: Arbeitserleichterung, logistischer Aufwand, (Integration in klinische Routine) und Patientenbelastung.

\subsubsection{Ergebnisse:}

\subsubsection{Zeitnahme}

Der Arbeitsablauf zur Durchführung einer mobilen CT im Interventionsraum der Intensivstation ist in Abbildung 5 in einem Gantt-Diagramm getrennt für die Humanressourcen Radiologe, Pflegepersonal und Intensivmediziner aufgeführt. Die Gesamtzeiten der Untersuchungen mit den beiden Methoden beliefen sich auf im Median 55 (47-76) Minuten für die mobile und 65 (51-84) Minuten für die ortsfeste. Im folgenden Text werden die Mediane der Untersuchungszeiten angegeben. Die Dauer der Transporte betrug für die mobile CT 3,1 Minuten und für die ortsfeste 9. Für letztere musste noch in eine Transferzeit differenziert werden, um der Notwendigkeit eines Transportdienstes zwischen Station und radiologischer Klinik aufzuzeigen. In 37 von 44 Fällen kam es zu Verzögerungen durch Wartezeiten auf den Transportdienst.

Die Erstellung des Topogramms mit der mobilen CT dauerte 7,9 Minuten, mit der ortsfesten 4,9 Minuten. Ebenso war die Zeit zur Bilddatenakquisition mit 4 Minuten mit der ortsfesten CT geringer als mit der mobilen, bei der im Median 6 Minuten vergingen (Abbildung 3-5). 


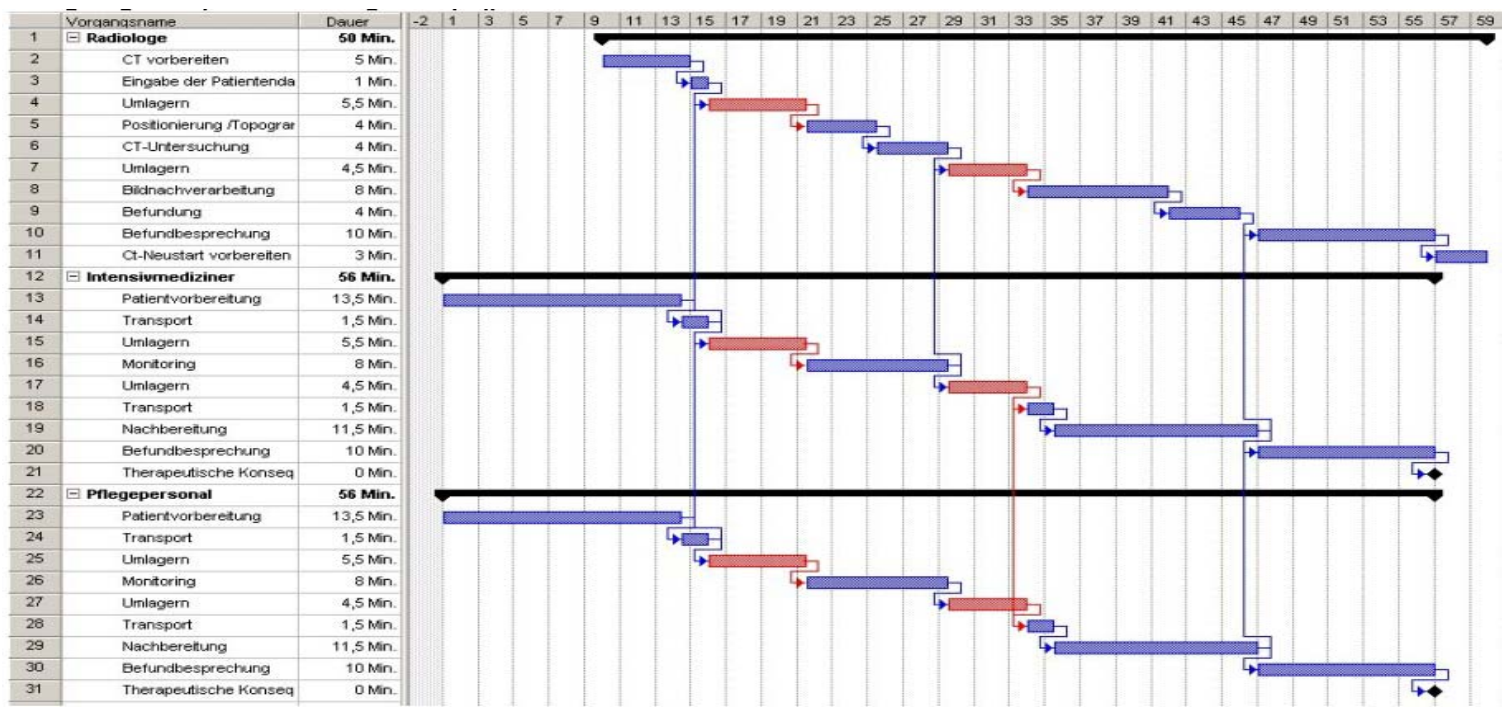

Abbildung 3-5: Gantt-Diagramm mit dem Arbeitsablauf zur Durchführung einer mobilen Computertomographie im Interventionsraum der Intensivstation.

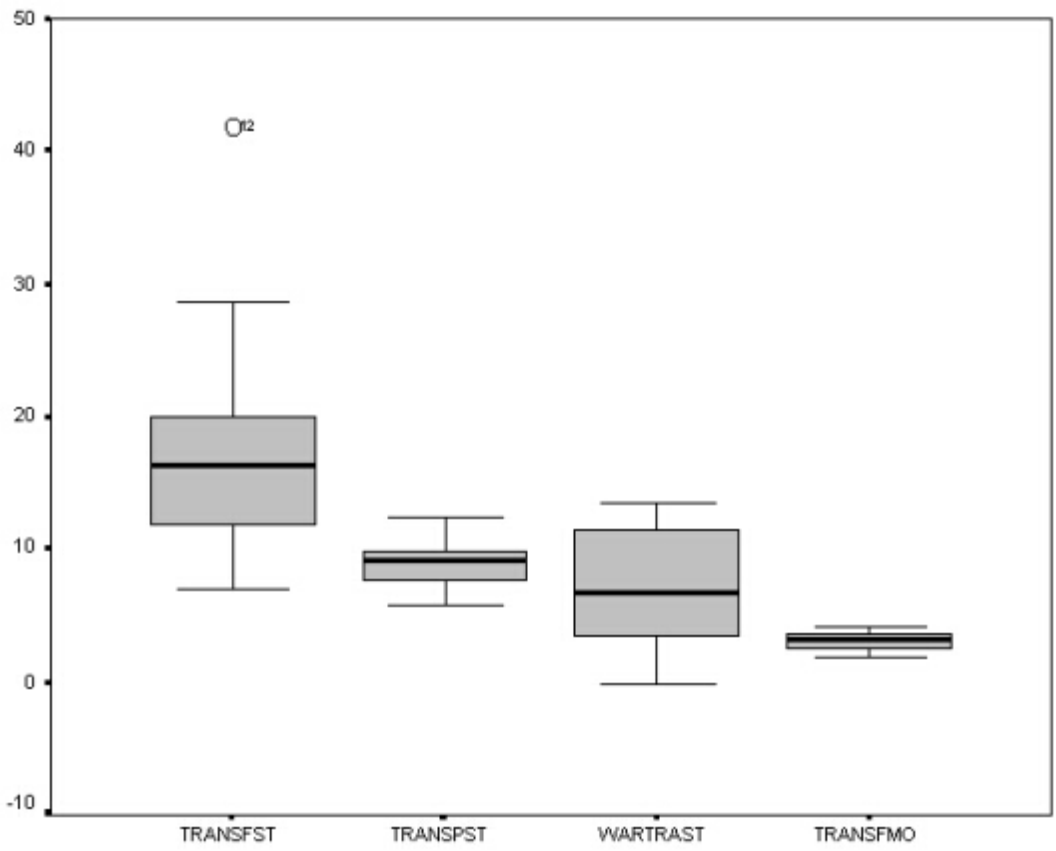

Abbildung 3-6: Transferzeiten von den Patientenzimmern zu den Untersuchungsräumen. 


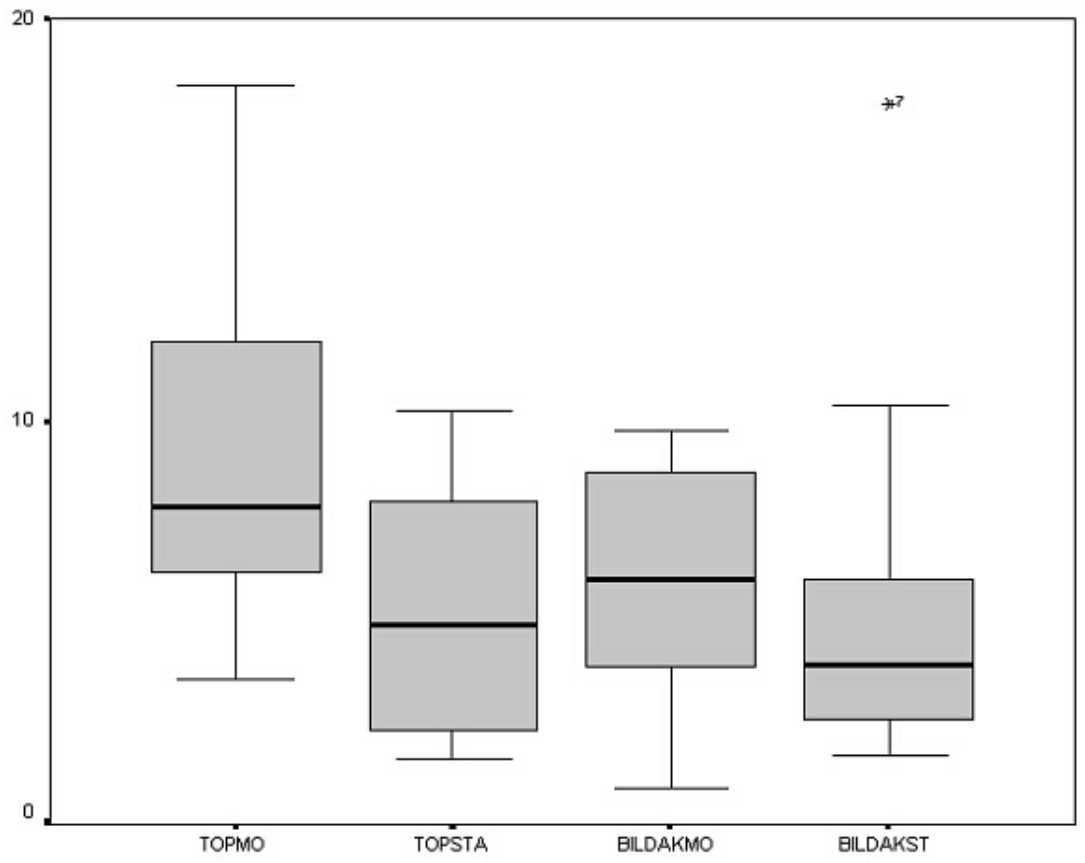

Abbildung 3-7: Zeitdauer der Topogrammerstellung und der Bildakquisition. 


\subsubsection{Korrelation der Entfernung und der Zeitmessung}

Die kürzeste Wegstrecke für den Transport zur mobilen CT lag bei 11 Metern, die längste bei 67 Metern für die Untersuchung im Interventionsraum. Die kürzeste Distanz von einem Intensivzimmer zur stationären CT betrug 68 Meter. Für die längste Wegstrecke von einem Intensivzimmer zur stationären CT waren 407 Meter zurückzulegen. Die Transportzeit stieg mit der Distanz in einem nahezu linearen Verhältnis an (Abbildung 3-8).

\section{Zeit-Entfernungskorrelation Radiol. Abteilung}

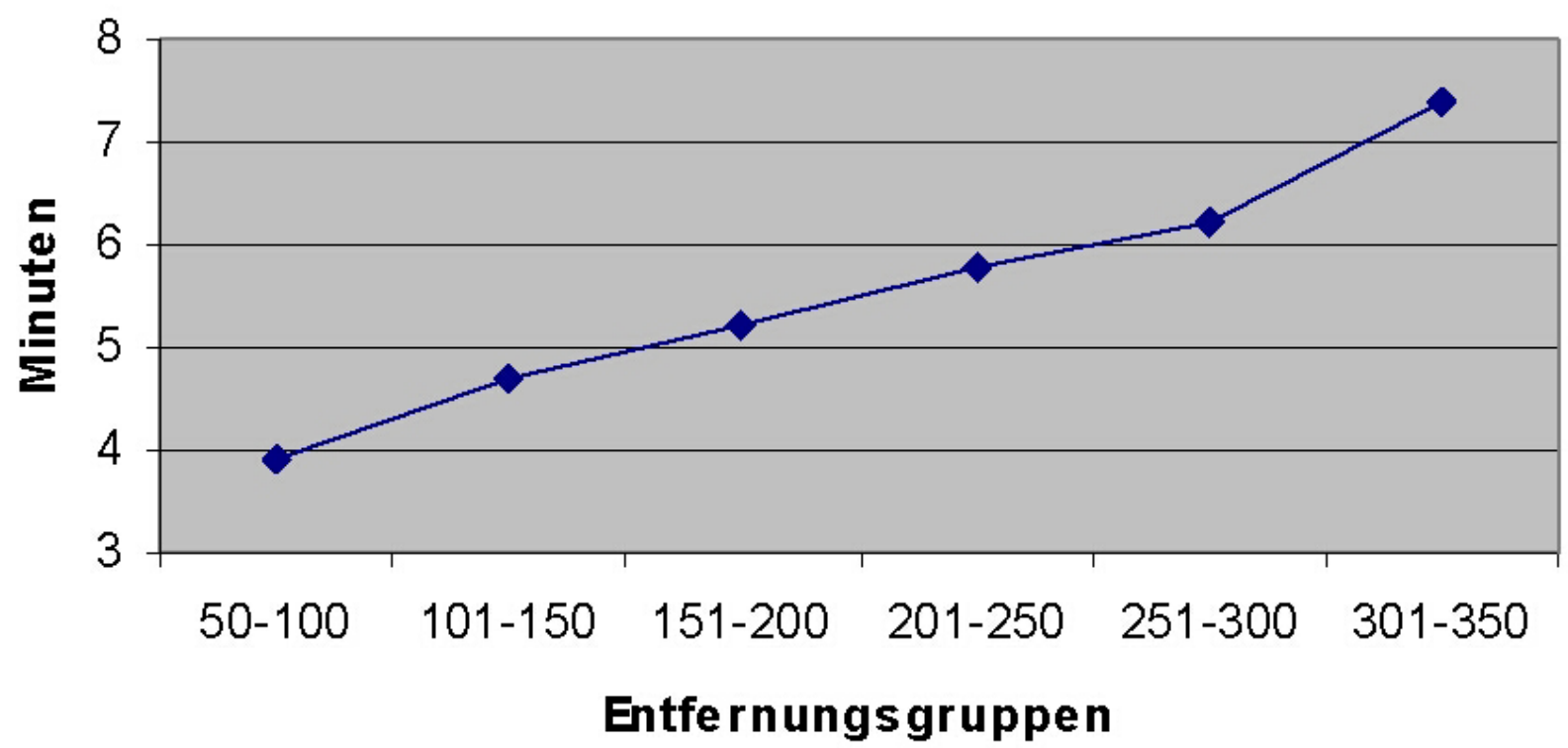

Abbildung 3-8: Zeit-Entfernungskorrelation für den Transport eines Patienten von der Intensivstation zur radiologischen Klinik. 


\subsubsection{Evaluation des intensivmedizinischen Personals}

Der Fragebogen zur Evaluierung der mobilen CT durch das Personal der Intensivstation wurde von 16 der 22 Ärzte und von 32 der insgesamt 75 Pflegekräfte ausgefüllt. Die mobile CT empfanden 13 der 16 Ärzte als eine Arbeitserleichterung gegenüber der stationären. Ein ähnliches Bild bot die Frage nach dem logistischen Aufwand für die mobile CT im Vergleich zur stationären. 14 der 16 Ärzte kamen zu dem Schluss, dass die mobile CT leichter plan- und durchführbar sei. Die Belastung für den Patienten bei der mobilen CT sei nach Ansicht von 10 Ärzten geringer als bei der stationären. Die CT auf der Intensivstation wird von 12 Ärzten favorisiert. 5 der befragten Ärzte bevorzugen jedoch weiterhin die Durchführung der CT-Untersuchung in der

Radiologischen

Klinik.

Die mobile CT wurde ebenso von den Pflegekräften in den genannten Punkten als überwiegend positiv bewertet.

\subsubsection{Diskussion}

Der zeitliche Gesamtaufwand von der mobilen und der stationären CT ist in etwa gleich. Dennoch gibt es erhebliche zeitliche Differenzen zwischen den Methoden, wenn man die Ergebnisse der Arbeitsschritte im einzelnen betrachtet. Wie zu erwarten war, ist die Transferzeit bei der mobilen CT (Median: 3,1 Minuten) deutlich kürzer als bei der ortsfesten CT (Median: 16,1), da es sich um einen Transport innerhalb der Intensivstation handelt. Diese Differenz war zum einen in den deutlich geringeren Distanzen für den Transport zur mobilen CT und im Fehlen von Hindernissen (Fahrstuhl) begründet. Zum anderen trugen die Wartezeiten auf den Transportdienst erheblich zu diesem Zeitunterschied bei (im Vergleich war der Median der Transferzeiten für die ortsfeste CT ohne zeitliche Verzögerungen 9 Minuten). Es ist schwierig einzuschätzen, welche Bedeutung die genannten Verzögerungen für den klinischen Tagesablauf hatten, zumal es sich jeweils nur um wenige Minuten handelte. Auf der Station konnte das Personal die Wartezeit mit anderen notwendigen Tätigkeiten überbrücken. Diese Option war im CTUntersuchungsraum in der radiologischen Klinik nicht gegeben. Die Durchführung der CT-Untersuchung (Topogrammerstellung und Bildakquisition) dauert mit der mobilen CT deutlich länger als mit der ortsfesten CT. Dies hat technische Gründe, da aufgrund der kompakten Bauweise der mobilen CT Kompromisse bei der technischen Ausstattung eingegangen werden mussten 
(längere Rotationszeit mit 2 Sekunden, Geringere Rechenkapazität der Workstation). Der geringe Unterschied der Gesamtuntersuchungszeit zugunsten der mobilen CT ist somit ausschließlich auf die unmittelbare Nähe zum Patienten zurückzuführen und dürfte in der Praxis kaum eine Bedeutung haben. Der Zeitpunkt der Untersuchung lässt sich bei der mobilen CT flexibler bestimmen. Hierbei bedarf es lediglich der Absprache des Stationspersonals mit dem Radiologen. Bei der ortsfesten CT in der Radiologischen Klinik muss der Termin zusätzlich mit den Bedürfnissen der anderen Stationen des Hauses abgestimmt werden. $\mathrm{Zu}$ bedenken ist jedoch auch, dass für die mobile CT ein Arzt aus der radiologischen Klinik zur Verfügung stehen muss. Der Radiologe betreibt bei uns die mobile CT selbständig ohne medizinisch-technische Assistenz und ist zusätzlich für die Stationssonographien verantwortlich. Dadurch lässt sich die mobile CT mit der sonographischen Arbeit auf den Intensivstation abstimmen. Für die Untersuchung mit der mobilen CT müssen der Patient und das Begleitpersonal die Intensivstation nicht verlassen. Treten Komplikationen auf, so kann unverzüglich auf das gesamte Spektrum der intensivmedizinischen Therapie zurückgegriffen werden (6;73). Ein weiterer Vorteil ist, dass das Begleitpersonal die Station für die Untersuchung nicht verlässt. Durch die mobile CT könnte demnach die Qualität der intensivmedizinischen Überwachung aller Patienten während der CTUntersuchung verbessert werden. In diesem Zusammenhang ist besonders darauf hinzuweisen, dass bei der mobilen CT die gesamte intensivmedizinische Überwachung und Medikation des Patienten während der Untersuchung beibehalten werden kann. Letzteres könnte sich vor allem bei kritischen Patienten als vorteilhaft erweisen. Bei Patienten mit Ventilationsstörungen ist die Beatmung mit Behelfsgeräten wie Oxylog, Medumat oder Ambubeutel nur kurzzeitig notwendig (während des Transports bei Untersuchung im Interventionsraum) oder kann sogar ganz entfallen (Untersuchung im Patientenzimmer). Diesen Geräten fehlen diverse Beatmungsoptionen, da sie nur für die kurzzeitige Beatmung bei Transport oder Reanimation außerhalb der Intensivstation konzipiert sind. Bestimmte Einstellungen der Ventilation sind aber für Patienten, die unter Oxygenierungsstörungen (z.B. ARDS) leiden, erforderlich $(7 ; 61 ; 63)$. Die mobile CT ermöglicht es weiterhin, alle über den zentralen Venenkatheter infundierten Medikamente auch während der Untersuchung zu applizieren. Für die stationäre CT war es ohne Ausnahme so, dass die Infusionsautomaten mit den nicht lebensnotwendigen Medikamenten diskonnektiert wurden. Die Möglichkeit zur CT-Untersuchung direkt auf der 
Intensivstation ermöglicht die Verwirklichung des AUDI-Prinzips (Aggressive Use of Diagnostic Imaging) (92; 95). Das Prinzip liegt im umfassenden und unverzüglichen Einsatz radiologischer Screeningverfahren bei neu aufgenommenen Patienten auf der Intensivstation. Die CT bietet gegenüber der konventionellen Röntgenaufnahme eine höhere Sensitivität für bestimmte auch lebensbedrohliche Gesundheitszustände (z.B. Pneumothorax, Hämothorax, Wirbelsäulenverletzungen). Dies trifft besonders bezüglich der notfallmäßig angefertigten ersten Röntgenaufnahmen zu (45). AUDI zielt darauf $a b$, frühzeitig die endgültige Diagnose zu stellen und dadurch den Patienten von Beginn an adäquat behandeln zu können. Durch die Verfügbarkeit der mobilen CT auf der Intensivstation kann die erweiterte Diagnostik und initiale Behandlung parallel durchgeführt werden. Ein weiterer Transport des Patienten durch das Krankenhaus wegen diagnostischer Untersuchungen wird damit überflüssig.

Ein weiterer wesentlicher Vorteil der mobilen CT ist die Möglichkeit der CTUntersuchung im Isolierzimmer oder der Isolierstation. Hierbei ist vor allem der Einsatz auf der onkologischen Intensivstation bemerkenswert. Patienten, die sich im Zustand nach Knochenmarkstransplantation befinden, müssen für einen gewissen Zeitraum isoliert werden. Aufgrund ihrer Immunsuppression in der Phase der Aplasie oder Neutropenie sind sie stark infektionsgefährdet (65). Gerade bei diesen Patienten ist, vor allem wenn sie klinische Entzündungszeichen bieten, eine frühzeitige, hochsensitive Diagnostik indiziert. Die CT bedeutet eine Verbesserung der Diagnostik, vor allem, da die konventionelle Röntgen-Thoraxaufnahme nur gering sensitiv für die Detektion von Lungeninfiltraten in der Frühphase nach Knochenmarktransplantation ist (97). Bei Infektionen, die zumeist den Respirationstrakt betreffen, oder sonstigen Komplikationen (Medikamententoxizität, Graft versus Host-Reaktion) kann somit rechtzeitig gezielt medikamentös therapiert werden. Bisher konnte auf der onkologischen Intensivstation nur die konventionelle Röntgenaufnahme unter sterilen Bedingungen im Isolierzimmer angefertigt werden. Bei weitergehenden Fragestellungen wurde eine CT-Untersuchung in der radiologischen Klinik unter eingehender Risikoabwägung durchgeführt. Hierfür musste die Isolierung für die Zeit der Untersuchung aufgehoben werden, was ein erhöhtes Infektionsrisiko für den Patienten bedeutete. Mit der mobilen CT auf der 
Intensivstation kann möglicherweise die Prognose bei isolierten Patienten in Zukunft verbessert

werden.

Die Evaluierung des intensivmedizinischen Personals ergab eine überwiegend positive Einschätzung der CT-Untersuchung auf der Intensivstation. Ähnliche Erfahrungen haben Mirvis et al. in ihrer Studie über die mobile CT gemacht (100). Die überwiegende Mehrzahl der behandelnden Ärzte und Pflegekräfte bewertete die mobile CT als eine Erleichterung für den Patienten und die eigene klinische Tätigkeit. Der logistische Aufwand für die Organisation der CT-Untersuchung ist ebenfalls nach Ansicht der Mehrzahl der befragten Personen bei der mobilen CT geringer als bei den bisherigen ortsfesten CT-Untersuchungen in der radiologischen Klinik. Die positive Einschätzung der mobilen CT wird auch dadurch unterstrichen, dass die Intensivstation gegenüber der radiologischen Klinik von der überwiegenden Zahl der Befragten als Untersuchungsort bevorzugt wird. Die genannten Einschätzungen sind bis jetzt nicht in Zahlen quantifizierbar, etwa anhand einer erniedrigten Morbidität oder Mortalität der untersuchten Patienten. Weitergehende Studien müssen in Zukunft klären, welchen quantifizierbaren Effekt die mobile CT auf das Wohl des Patienten

besitzt.

Zusammenfassend lässt sich festhalten, dass sich der Einsatz der mobilen CT auf der Intensivstation bewährt hat. Für den überwiegenden Teil der intensivpflichtigen Patienten bevorzugen wir eine Durchführung der Untersuchung in einem Interventionsraum unmittelbar auf der Intensivstation. Die Unterschiede der mobilen zur stationären CT liegen bei dieser Methode in der kürzeren Transportzeit und dem Verbleiben des Patienten sowie des begleitenden Intensivmediziners auf der Intensivstation. Letzteres gewährleistet das ständige Vorhandensein der gesamten intensivmedizinischen Ausrüstung, das bei Bedarf zur Behandlung des Patienten in vollem Umfang zur Verfügung steht. Daraus resultiert die hohe Akzeptanz der mobilen CT beim intensivmedizinischen Personal. Die Durchführung der CTUntersuchung direkt im Patientenzimmer bleibt nur Patienten vorbehalten, bei welchen ein Transport unmöglich ist (IAPP, Extrakorporale Lunge, Dialyse). Das Aufstellen der mobilen CT direkt im Patientenzimmer ist mit einem erheblichen zeitlichen Aufwand verbunden und aus Strahlenschutzgründen den anderen im gleichen Zimmer befindlichen Patienten nicht zuzumuten $(71$; 92). Nach Bedarf kann die mobile CT-Einheit auch an anderen Einsatzorten innerhalb des Krankenhauses wie anderen Intensivstationen, OP, Angiographie oder Notaufnahme zur Verfügung 
stehen. Durch die hohe Flexibilität kann die mobile CT insbesondere bei großen Kliniken sinnvoll ausgelastet werden. 


\section{Anwendungen der Netzplantechnik \\ 4.1 Netzplantechnik zur Zeitplanung bei abdominellen Ultraschalluntersuchungen}

\subsubsection{Einleitung}

Die Organisation und zeitliche Bemessung von diagnostischen Untersuchungsmethoden ist insbesondere unter dem zunehmenden Kostendruck in Kliniken und Arztpraxen ein zentrales Thema. In der vorliegenden Studie sollen Maßnahmen aus dem Bereich des Prozessmanagement vorgestellt werden, wie sie auch vom Arzt in seiner klinischen Praxis angewendet werden können. Die Methoden der Netzplantechnik (NPT) kommen vor allem bei der Produktionsplanung und -durchführung zur Anwendung. Die Netzplantechnik umfasst die Verfahren der Durchführungsplanung (und -kontrolle ) von Projekten auf der Grundlage von Netzplänen. In diesem Zusammenhang wird unter einem Projekt ein abgrenzbares Vorhaben mit einer komplexen Realisierungsplanung verstanden. In der vorliegenden Studie repräsentiert die Ultraschalluntersuchung das Projekt. Bei der Durchfühungskontrolle werden die einzelnen Realisierungsmaßnahmen näher betrachtet. Bei der Ultraschalluntersuchung repräsentieren die einzelnen Arbeitsschritte die Realisierungsmaßnahmen. Die NPT ist somit ein wichtiges Instrument bei der Organisationsplanung. Einzelne Arbeitsschritte werden bei der NPT auf der Grundlage von Netzplänen dargestellt. Mit Netzplänen lassen sich komplexe Arbeitsabläufe zur Darstellung bringen, Engpassanalysen durchführen und Arbeitsabläufe kontrollieren. Die wichtigsten Verfahren der Netzplantechnik sind die "Critical Pathway Method“ (CPM), „Program Evaluation and Review Method" (PERT) und "Metra Potential Method" (MPM). Aus diesen fast gleichzeitig entstandenen Verfahren der NPT haben sich im Laufe der Zeit über 50 abgeleitete Verfahren entwickelt. Die CPM wurde Ende der 50er und Anfang der 60er Jahre von Morgan Walker und James Kelly entwickelt und kam bei den Firmen Du Pont und Remington Rand erstmals zur Anwendung. Insbesondere die CPM hat sich wegen ihrer Übertragbarkeit und universalen Anwendbarkeit für den Bereich der klinischen Medizin gegenüber anderen Netzplantechniken durchgesetzt. Mit der CPM lassen sich Struktur-, Zeit-, Kosten- und Beschäftigungsbzw. Kapazitätsplanungen durchführen. In der vorliegenden Studie wurde der Versuch unternommen, die NPT auf die Durchführung der Ultraschalluntersuchung anzuwenden. 


\subsubsection{Methode}

Zur Erstellung eines CPM-Netzplans für B-Bild Ultraschalluntersuchungen soll eine Strukturplanung in drei Phasen durchgeführt werden. Die Ultraschalluntersuchung entspricht dabei dem durchzuführenden Projekt und die Ereignisse entsprechen den Arbeitsschritten der Ultraschalluntersuchung. In die Betrachtungen werden nur abdominelle B-Bild Ultraschalluntersuchung ohne Einsatz von Farb- oder Duplexsonographie eingeschlossen. Als Patienten dienten mobile Patienten, welche entweder von einer onkologischen Station, der onkologischen Tagesklinik oder ambulant zur Ultraschalluntersuchung kamen. Bettlägerige Patienten wurden bei den Messungen nicht berücksichtigt. Die abdominelle Ultraschalluntersuchungprotokoll beinhaltet die folgenden Untersuchungsgebiete: Leber, Gallenblase, Milz, Pankreas, Nebennierenregion, Nieren, Retroperitoneum (Suche nach Lymphomen), große abdominelle Gefäße, Blase, gegebenenfalls Prostata oder Uterus. Es befanden sich zwei Ultraschallgeräte, ein Toshiba SSA 340A (Toshiba Tokio, Japan) und ein Toshiba SSA 100A (Toshiba, Tokio, Japan) im Einsatz. Für die abdominellen Ultraschalluntersuchungen wurde ein Curved-Array Schallkopf $3,5 \mathrm{MHz}$ verwendet. Die Ultraschallgeräte befanden sich in zwei getrennten Untersuchungsräumen, die schriftliche Befunderstellung erfolgte in einem separaten Raum an einem PersonalComputer. Im Funktionsbereich Ultraschall arbeiten jeweils zwei Ärzte und eine Krankenschwester. Die Ärzte arbeiteten an beiden Geräten alternierend. Die bei den Zeitmessungen berücksichtigten Ärzte waren fachlich qualifiziert mit langjähriger Ultraschallerfahrung und besitzen den Status eines DEGUM Ausbilders. In der ersten Phase der Strukturplanung wurde das Projekt (abdominelle USUntersuchung) in einzelne Ereignisse (Arbeitsschritte) zerlegt. Hierzu haben zwei Ärzte die Anzahl von 10 Ultraschalluntersuchungen als neutrale Beobachter begleitet und die Arbeitsschritte definiert. Die Arbeitsschritte wurden in einer Ereignisliste zusammengeführt. Jedem Arbeitsschritt wurde dabei ein Buchstaben zugewiesen. In der Ereignisliste wurden alle vorkommenden Arbeitsschritte mit Zeitdauer und gegenseitiger Abhängigkeit eingetragen. In der zweiten Phase der Strukturplanung wurden die Arbeitsschritte in eine Vorgänger-Nachfolger-Beziehung gebracht. Dies erfolgte durch ein Beziehungsschema, in welchem die Beziehungen der einzelnen Arbeitsschritte zueinander in graphischer Form dargestellt wurden. Das Beziehungsschema der Arbeitsschritte zeigt in der Horizontalen die zu betrachtenden Ereignisse, die jeweils dann beginnen können, wenn die anderen in der Vertikalen angeordneten Ereignisse abgeschlossen sind. Betrachtet man zwei Arbeitsschritte 
(Ereignisse), z.B. Ereignis $A=$ Untersuchung des Patienten durch den Arzt und Ereignis $\mathrm{B}=$ Erstellung des Befundes durch den Arzt, so können folgende 4 Beziehungsarten unterschieden werden:

1. Ende-Start-Beziehung $=B$ erst beginnen, wenn $A$ beendet ist.

2. Start-Start-Beziehung $=B$ erst beginnen, wenn $A$ begonnen hat.

3. Start-Ende-Beziehung $=B$ erst beenden wenn $A$ begonnen hat.

4. Ende-Ende-Beziehung $=B$ erst beenden, wenn $A$ beendet ist.

Im weiteren wurden in der zweiten Phase die Zeitmessungen der zuvor definierten Arbeitsschritte durchgeführt. Es wurden insgesamt bei 30 abdominellen Ultraschalluntersuchungen die einzelnen Arbeitsschritte mit der Stoppuhr gemessen. Durch die Zeitmessung erfolgte eine Bestimmung der Vorgangs- und Verknüpfungsdauern der Arbeitsschritte, bzw. die Wahrscheinlichkeitsverteilung dieser Zeiten. In der dritten Phase der Strukturplanung wurden zunächst an Hand des Beziehungsschemas die parallel durchführbaren Arbeitsschritte ermittelt. Dann erfolgte die Abbildung des Projektes durch einen pfeilorientierten Netzplan. Der Arbeitsvorgang wurde hierbei als Pfeil und der eigentliche Arbeitsschritt (Ereignis) als Knoten dargestellt (Abbildung 4-1). Es werden anhand der in der Phase 2 erhobenen Zeitmessungen die jeweils frühsten („early start" = ES) und spätesten Zeitpunkte für den Eintritt des Ereignisses (,early finish“ = EF) bestimmt. Darüber hinaus wird der früheste ("late start“ = LS) und späteste Zeitpunkt („late finish“ = LF) für die Beendigung eines Ereignisses bestimmt. Diese Daten werden für jeden Verknüpfungspunkt (Arbeitsschritt) angegeben. Die Verknüpfung der einzelnen Arbeitsschritte erfolgt nach den oben genannten feststehenden Regeln. Der Netzplan beginnt genau mit einem Startpunkt (Quelle) und genau einem Endpunkt (Senke). Der Start der Ultraschalluntersuchung wurde mit dem Abruf des Patienten aus dem Wartebereich definiert und das Ende mit dem Aushändigen des schriftlichen Befundes festgelegt. Endeten oder begannen in einem Arbeitsschritt mehrere Vorgänge, die nicht alle voneinander abhängig waren, wurden zur Darstellung der Zusammenhänge Scheinvorgänge benutzt. Scheinvorgänge sind fiktive Vorgänge mit einer Zeitdauer von 0 und dienen der Darstellung von logischen Verknüpfungen. 


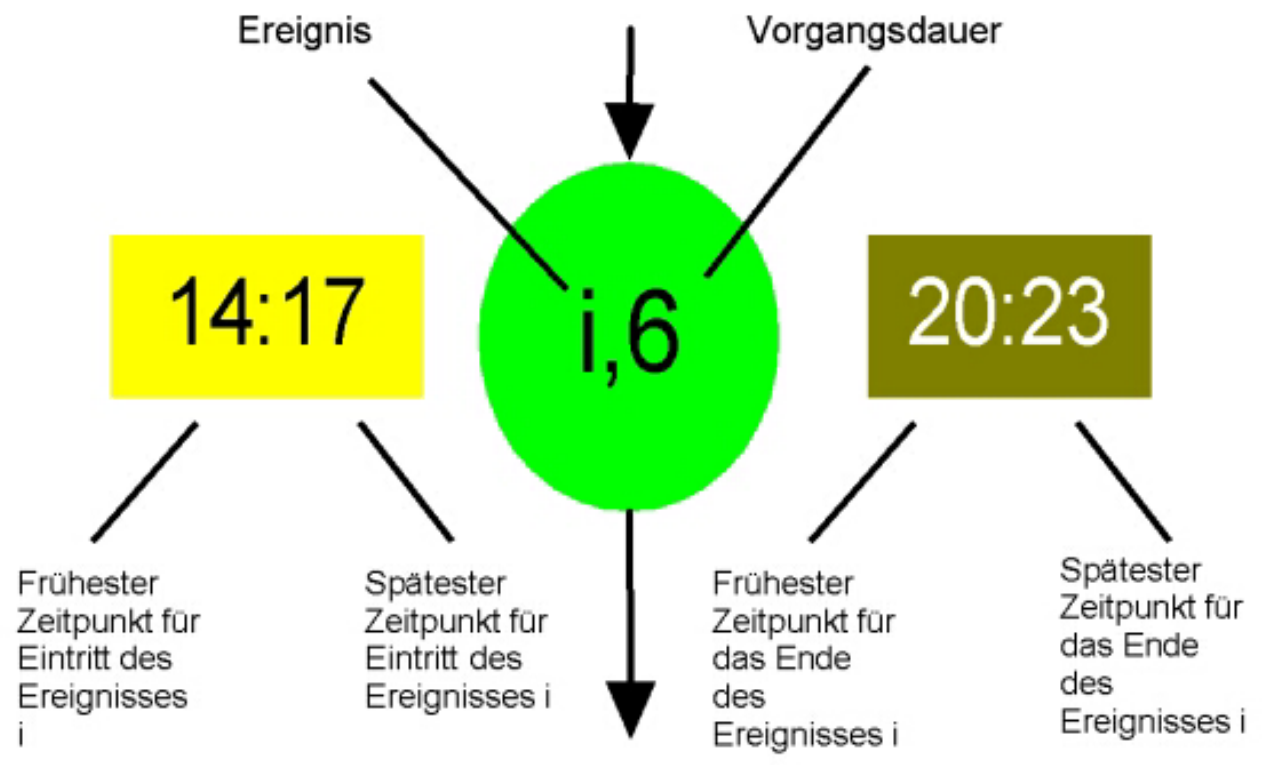

Abbildung 4-1: Beschreibung der Daten eines Arbeitschrittes im Netzplan.

Auf der Grundlage des Netzplanes wurden die Gesamtprojektdauer und die Zeitreserven bestimmt. Unter den Zeitreserven werden dabei die Puffer- und Schlupfzeiten der Ultraschalluntersuchung verstanden. Die Pufferzeit gibt den Zeitraum an, um welchen ein Arbeitsschritt verlängert werden kann, ohne dass die Gesamtuntersuchungsdauer überschritten wird. Vorgänge mit einer Pufferzeit von 0 sind kritische Vorgänge, d.h. diese Arbeitsschritte besitzen keine Zeitreserven. Es können die Gesamtpufferzeit (GP), die freie Pufferzeit (FP) und die unabhängige Pufferzeit (UP) unterschieden werden. Die FP ist der Zeitraum, um den die Vorgangsdauer erhöht werden kann, wenn alle Vorgänge zu den geplanten frühesten Anfangszeitpunkten (FAZ) beginnen. Die UP beschreibt den Zeitraum, um den die Vorgangsdauer eines Arbeitsschrittes erhöht werden kann, wenn alle vorausgegangenen Arbeitsschritte zu den geplanten spätesten Anfangszeiten und alle nachfolgenden Vorgänge zu den geplanten frühesten Anfangszeiten beginnen. Die Pufferzeiten der einzelnen Arbeitsschritte werden dadurch berechnet, dass von ihrem ES-Wert der dazugehörende EF-Wert subtrahiert wird (FP = ES - EF). Es wurden die Zeitreserven einer abdominellen Ultraschalluntersuchung bestimmt. Die minimale Zeit für die Durchführung einer abdominellen Ultraschalluntersuchung wurde gemessen und auf dieser Grundlage der kritische Weg der Ultraschalluntersuchung bestimmt. 


\subsubsection{Ergebnisse}

Es wurde die abdominelle Ultraschalluntersuchung nach einer unabhängigen Beobachtung in einzelne Ereignisse (Arbeitsschritte) zerlegt und in Tabelle 4-1 zusammengefasst. Insgesamt wurden 14 Arbeitsschritte definiert ohne den Anfang und Endpunkt als Arbeitsschritt zu werten.

Tabelle 4-1: Arbeitsschritte der abdominellen Ultraschalluntersuchung

\begin{tabular}{|c|c|c|c|}
\hline Ereignis & Beschreibung & Vorgänger & $\Delta \mathrm{t}[\mathrm{min}]$ \\
\hline$A$ & Quelle & & 0 \\
\hline$B$ & Abruf des Patienten & $A$ & 0,5 \\
\hline C & $\begin{array}{ll}\text { Aufnahme } & \text { der } \\
\text { Patientendaten } & \end{array}$ & B & 3 \\
\hline D & Lagerung des Patienten & C & 1 \\
\hline$E$ & Kurzanamnese & $D$ & 2 \\
\hline $\mathrm{F}$ & Ultraschalluntersuchung & $E$ & 6 \\
\hline G & Akte anlegen & $\mathrm{F}$ & 2 \\
\hline $\mathrm{H}$ & $\begin{array}{l}\text { Abruf des nächsten } \\
\text { Patienten }\end{array}$ & $G$ & 0,5 \\
\hline I & $\begin{array}{l}\text { Aufnahme des } \\
\text { nächsten Patienten }\end{array}$ & $\mathrm{H}$ & 3 \\
\hline $\mathrm{J}$ & $\begin{array}{l}\text { Lagerung des nächsten } \\
\text { Patienten }\end{array}$ & 1 & 1 \\
\hline $\mathrm{K}$ & Säuberung & $F, j$ & 2,5 \\
\hline $\mathrm{L}$ & Verabschiedung & $F$ & 2,5 \\
\hline$M$ & Dokumentation & $\mathrm{L}$ & 6 \\
\hline$N$ & Akte vervollständigen & $\mathrm{K}, \mathrm{m}$ & 2 \\
\hline $\mathrm{O}$ & Versand & $N$ & 1 \\
\hline$p$ & Senke & $\mathrm{O}$ & 0 \\
\hline
\end{tabular}




\subsubsection{Phase der Strukturplanung}

In der Abbildung 4-1 ist das Beziehungsschema von Vorgänger-NachfolgerBeziehungen der einzelnen Arbeitschritte aufgezeigt. Die in der Vertikalen befindlichen Arbeitsschritte können bis auf zwei Ausnahmen beginnen, wenn der unmittelbar vorherige Arbeitsschritt abgeschlossen ist. Das Ereignis k (Säuberung des Patienten) kann erst erfolgen, wenn die Arbeitsschritte $f$ (Ultraschalluntersuchung) und $\mathrm{j}$ (Lagerung des nächsten Patienten) abgeschlossen sind. Der Vorgang von $f$ nach $j$ entspricht einem Scheinvorgang, da dieser Vorgang keiner wirklichen Tätigkeit entspricht, jedoch eine Bedingung für den Beginn des Arbeitsschrittes $k$ darstellt. Die Ergebnisse der Zeitmessung an 30 Ultraschalluntersuchungen sind in Tabelle 3-1 als die jeweils kürzest möglichen Zeiten für die Durchführung der einzelnen Arbeitsschritte angegeben. Dies entspricht der Angabe der kürzesten Vorgangsdauer.

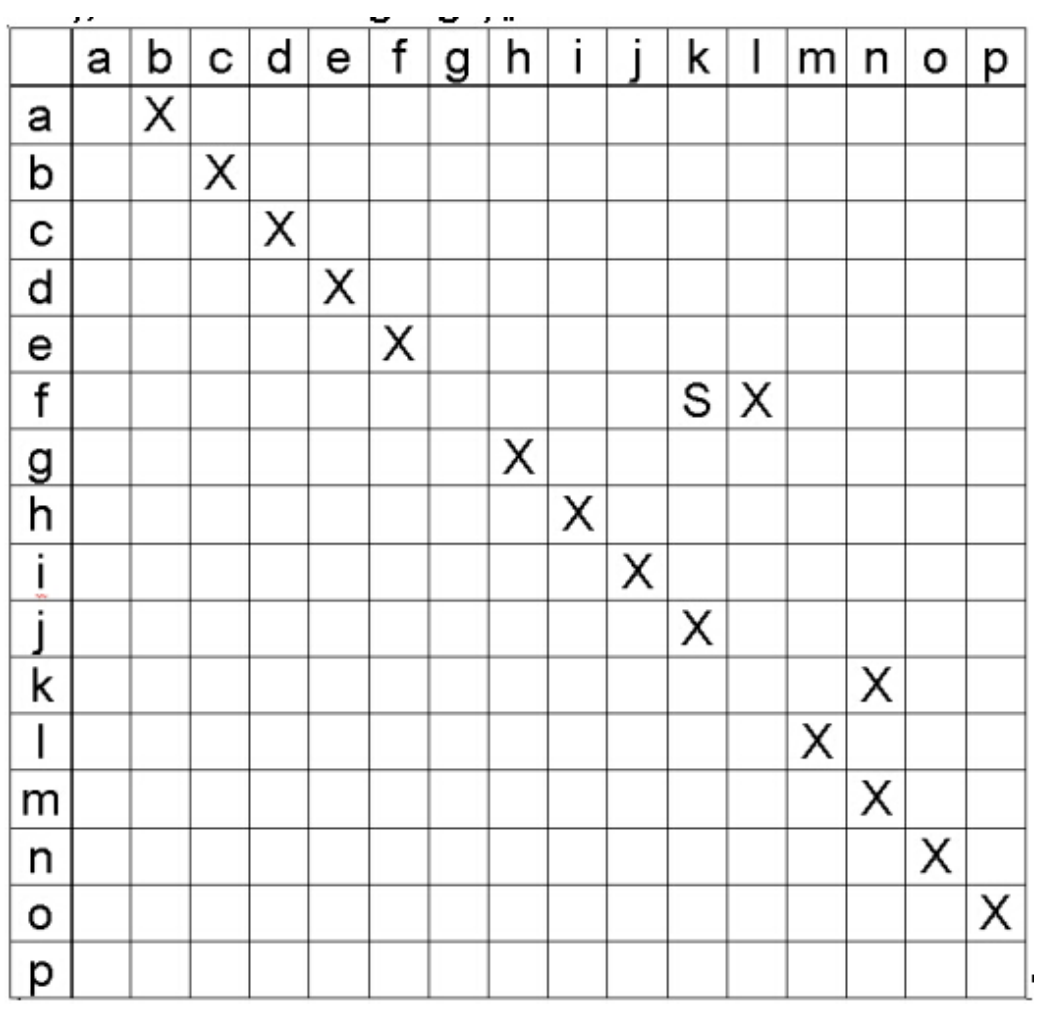

Abbildung 4-2: Beziehungsschema der Arbeitsschritte der abdominellen Ultraschalluntersuchung ( $X=$ Vorgänge (Arbeitsschritte), $S=$ Scheinvorgänge)

Im Beziehungsschema werden die Vorgänger-Nachfolger-Beziehungen der Arbeitsschritte aufgezeigt. Das Beziehungsschema zeigt in der Horizontalen die zu 
betrachtenden Ereignisse, die jeweils dann beginnen können, wenn die anderen, in der vertikalen angeordneten Ereignisse abgeschlossen sind. Scheinvorgänge sind fiktive Vorgänge mit einer Zeitdauer von 0 und dienen der Darstellung von logischen Verknüpfungen.

In Abbildung 4-3 ist der auf den Ergebnissen der Phase 1 und 2 erstellte Strukturplan für die Durchführung einer abdominellen Ultraschalluntersuchung dargestellt. Aus dem Strukturplan ergibt sich eine kürzest mögliche Zeit von 24 Minuten für die Durchführung einer kompletten abdominellen Ultraschalluntersuchung (Gesamtprojektdauer) einschließlich der Vor- und Nachbereitung des Patienten und die Erstellung des schriftlichen Befundes. Für die eigentliche Ultraschalluntersuchung durch den qualifizierten Facharzt werden dabei mindestens 6 Minuten benötigt. In unserer Klinik beträgt die Zeitvorgabe 40 Minuten für die Durchführung. Diese Zeitvorgabe ist im Netzplan berücksichtigt und wurde für den gesamten Netzplan ausgehend von der Senke rückwärts gerechnet. Um die Gesamtprojektdauer von 24 Minuten einhalten zu können, müssen die Arbeitsschritte a, b, c, d, e, f, I, m, n, o in der kürzest möglichen Zeit durchgeführt werden. Dies entspricht dem kritischen Weg. Die Arbeitsschritte des kritischen Weges besitzen keine FP, wenn diese zum FAZ beginnen. Die Arbeitsschritte des Arztes befinden sich alle auf dem kritischen Pfad. Somit bestehen für den Arzt keine Zeitreserven bei der Untersuchung. Anders verhält es sich bei der Krankenschwester: Die Arbeitsschritte g, h, i, j und k befinden sich nicht auf dem kritischen Pfad. Es bestehen somit für die Krankenschwester vor Beginn des Arbeitschrittes Säuberung ( $k$ ) eine freie Pufferzeit von 1,5 Minuten und anschließend nach Beendigung der Säuberung besteht eine weitere freie Pufferzeit von insgesamt 6 Minuten, ohne dass die Gesamtprojektzeit gefährdet ist. Somit ergibt sich eine Gesamtzeitreserve von 7,5 Minuten für die Krankenschwester bei einer Ultraschalluntersuchung. 


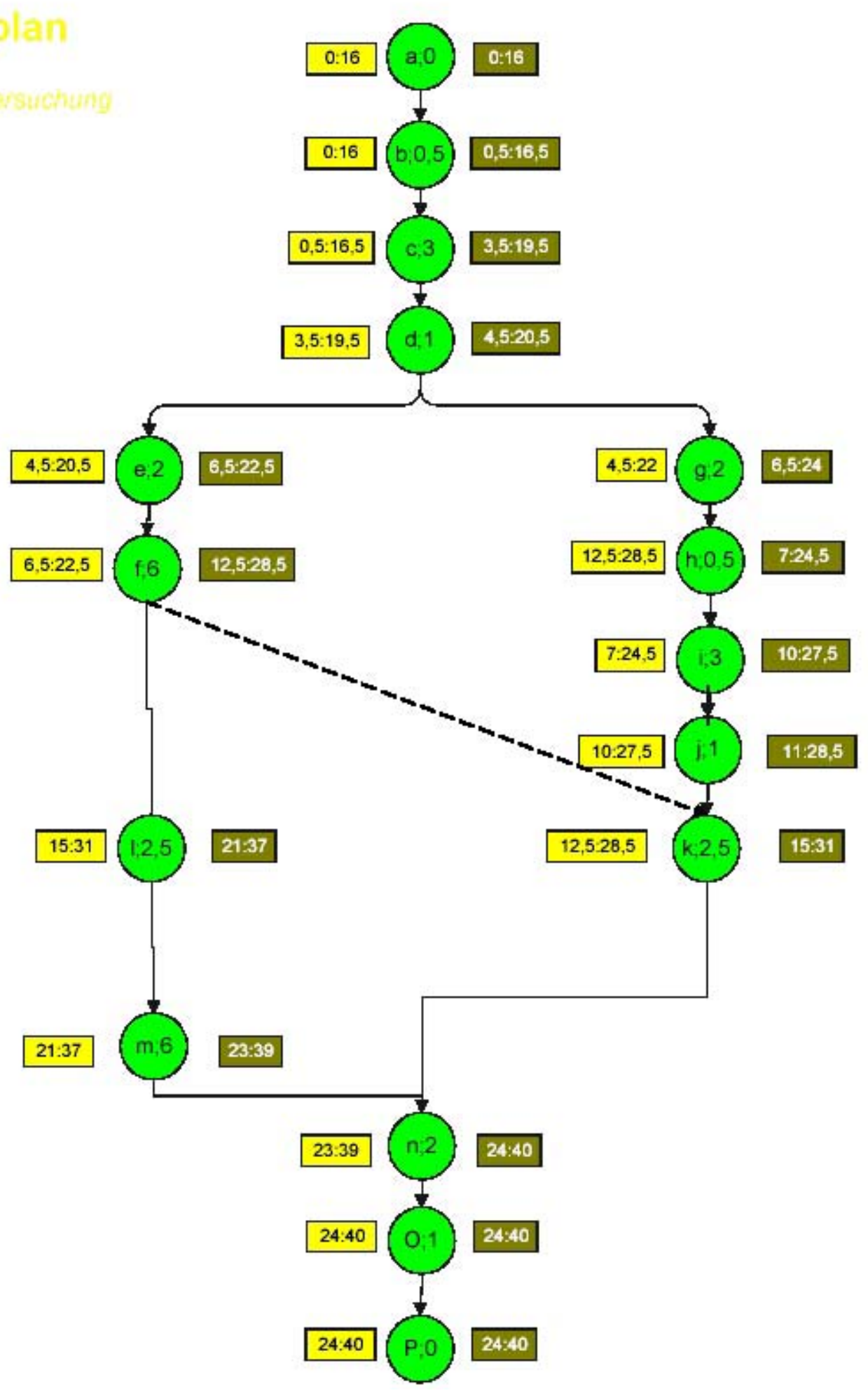

Abbildung 4-3: Strukturplan abdominelle Ultraschalluntersuchung. 


\subsubsection{Diskussion}

Die Netzplantechnik ermöglicht in anschaulicher Weise, wie viel Zeit für einzelne Arbeitschritte bezogen auf einen Arbeitsprozess benötigt wird und in welchen Beziehungen die einzelnen Arbeitschritte zueinander stehen. Somit ist der minimale Zeitbedarf für die Durchführung einer abdominellen Ultraschalluntersuchung ohne Verwendung des Farbdopplers 24 Minuten. Diese Zeitspanne beinhaltet die Vorbereitungs- und Nachbereitungszeit. Für die reine Ultraschalluntersuchung am Gerät werden vom Arzt mindestens 6 Minuten benötigt. Die eigentliche Ultraschalluntersuchung (mit dem Schallkopf am Patienten) beansprucht mindestens $25 \%$ von der Gesamtuntersuchungszeit. Die schriftliche Befundtexterstellung erfolgt unmittelbar nach Abschluss der Ultraschalluntersuchung und beansprucht weitere 6 Minuten. Der Arzt führt 4 von insgesamt 14 Arbeitsschritten selbst durch. Der Arzt muss insgesamt mindestens 16,5 Minuten für eine Untersuchung aufwenden. Dies beinhaltet eine Kurzanamnese von 2 Minuten, die Ultraschalluntersuchung, das kurze Abschlussgespräch von mindestes 2,5 Minuten und die anschließende Dokumentation am Computer. Alle Arbeitsschritte des Arztes sind „kritisch“ zu bewerten, d.h. eine eventuelle Zeitüberschreitung eines Arbeitsvorganges des Arztes würde die Gesamtzeit der Ultraschalluntersuchung verlängern. Aus dem Netzplan in Abbildung 4-3 ergibt sich, dass der kritische Pfad durch die Arbeitsschritte a, b, c, d, $\mathrm{e}, \mathrm{f}, \mathrm{I}, \mathrm{m}, \mathrm{n}, \mathrm{o}$ und $\mathrm{p}$ definiert ist. Die vier Arbeitsschritte des Arztes (e, f, I, m) befinden sich alle auf dem kritischen Pfad. Als Konsequenz daraus ergibt sich, dass für den Arzt keine Pufferzeiten im Sinne von Pausen bezogen auf eine Untersuchung bestehen. Wenn der Arzt Pausen während einer Untersuchung einlegen würde, hätte dies automatisch zur Folge, dass sich die Gesamtuntersuchungszeit von 24 Minuten verlängern würde. Pausen zwischen den Untersuchungen hätten weiterhin zur Folge, dass sich die Gesamtzahl der an einem Arbeitstag durchgeführten Untersuchungen verringern würde. Ungewollte Pausen finden beispielsweise schon durch Telephonate, welche der Arzt eventuell beantworten muss statt. Auch ein komplizierter Casus kann zu einer deutlichen Verlängerung der Gesamtuntersuchungszeit führen. Bei der Planung von Untersuchungskapazitäten muss dies berücksichtigt werden. Die Arbeitsschritte der Krankenschwester befinden sich nicht alle auf dem „kritischen Pfad“. Für die Krankenschwester bestehen bezogen auf eine Untersuchung Zeitreserven von insgesamt 7 Minuten. Dieses Zeitintervall bezieht sich auf die minimale Gesamtuntersuchungszeit von 24 Minuten. Bei Verzögerungen der Ultraschalluntersuchung, die durch den Arzt hervorgerufen 
werden, erhöhen sich sogar die Pufferzeiten der Krankenschwester. Die Krankenschwester besitzt bei einem Einsatz im Ultraschallfunktionsbereich somit genügend freie Kapazitäten, welche bei der Arbeitsplanung berücksichtigt werden müssen. Die Krankenschwester könnte neben der Vor- und Nachbereitung der Ultraschalluntersuchung noch weitere Tätigkeiten ausführen, ohne dass es zu einer Beeinträchtigung bei der Ultraschalluntersuchung käme. Zwischen den Arbeitsschritten Ultraschalluntersuchung (Arzt) und Säuberung des Untersuchungsraums (Krankenschwester) besteht eine Scheintätigkeit. Die Scheintätigkeit ist eine fiktive Tätigkeit, hat eine Zeitdauer von 0 und dient ausschließlich zur Darstellung logischer Verknüpfungen: Die Ultraschalluntersuchung muss erst beendet sein, bevor die Krankenschwester mit der Säuberung des Untersuchungsraums beginnen kann. Ohne die Verwendung von Scheintätigkeiten (gestrichelte Linie in Abbildung 4-3) wäre eine Berechnung der Zeitreserven nicht zuverlässig möglich. Unsere Studienergebnisse sind mit denen von Reuß et al. vergleichbar (72), welche in zwei Umfragen in Kliniken und Arztpraxen den Zeitaufwand für Ultraschalluntersuchungen erfasst haben. Für Kliniken ergab sich dabei ein Gesamtzeitaufwand von durchschnittlich 21,7 Minuten für die Durchführung einer Ultraschalluntersuchung. Die reine Untersuchungszeit (mit dem Schallkopf am Patienten) betrug für den sonographischen Oberbauchstatus durchschnittlich 12,4 Minuten. Bei den eigenen Messungen wurde hierfür eine minimale Untersuchungszeit von 6 Minuten bestimmt. Aus unserer praktischen Erfahrung ist eine durchschnittliche Zeit von 12 Minuten reiner Untersuchungszeit ein realistisches Zeitintervall. Bei der CPM finden Durchschnittszeitwerte jedoch keine unmittelbare Berücksichtigung.

Unsere Ergebnisse zeigen deutlich, dass die von der Kassenärztlichen Vereinigung (KV) veranschlagten 6 Minuten für die reine Untersuchungshaft gerade der minimalen Untersuchungszeit (mit dem Schallkopf am Patienten) unter optimalen Bedingungen entspricht (2). Es sollte jedoch bei einer Kostenerstattung nicht von minimalen Untersuchungszeiten sondern von Durchschnittswerten ausgegangen werden. Diese Zeitwerte sind bei der unmittelbaren Ultraschalluntersuchung mit höheren Werten als 6 Minuten zu veranschlagen. Darüber hinaus müssen auch die Vor- und Nachbereitungszeiten bei der Ultraschalluntersuchung, welche $75 \%$ der Gesamtuntersuchungszeit beanspruchen, mit angerechnet werden, so dass in den 
Abrechnungssystemen der gesamte Zeitbedarf für die diagnostische Untersuchung Berücksichtigung finden sollte. $\mathrm{Zu}$ beachten ist auch, dass sich durch eine verbesserte Ultraschallgerätetechnik über die letzten 15 Jahre nicht nur die diagnostische Qualität, sondern auch die Untersuchungszeiten wesentlich verringert haben. So ist man 1985 noch von einem Zeitbedarf von 30-40 Minuten für einen Oberbauchstatus ausgegangen (57). Heutzutage erscheint ein Zeitbedarf von 6 bis 10 Minuten als realistisch.

Die in unserer Studie aufgezeigten Zeitwerte lassen sich nicht uneingeschränkt auf andere Kliniken oder eine private Arztpraxis transferieren. Allerdings stellt unserer Ultraschallfunktionsbereich eine typische Konstellation dar, wie sie auch in anderen Kliniken vorzufinden ist. Keine Berücksichtigung fand bei unseren Zeitmessungen, dass auch Nicht-Fachärzte oder Ärzte, die in der Ultraschallausbildung befindlich sind, Untersuchungen durchführen. In diesen Fällen muss ein Oberarzt (in unserem Fall ein DEGUM Ausbilder) die Untersuchungsergebnisse verifizieren. Dies ist mit einem nicht unerheblichen Zeitaufwand verbunden, der vielfach die eigentliche Untersuchungszeit übersteigt. Insbesondere in Universitätskliniken ist dies ein wesentlicher Faktor, der berücksichtigt werden muss. Die im Netzplan angeführten Vorgangsdauern entsprechen jeweils dem minimalen Zeitintervall für die Durchführung eines Arbeitsschrittes. Deshalb muss noch einmal betont werden, dass die Zeit von 24 Minuten der geringst möglichen Zeitspanne entspricht, in der die komplette Untersuchung durchgeführt werden kann. Eine weitere Verkürzung der Untersuchungszeit hätte einen deutlichen Qualitätsverlust zur Folge. Es muss natürlich anhand des Netzplanes geprüft werden, ob die einzelnen Arbeitsschritte wie auch der Arbeitsalgorithmus noch Optimierungspotential bieten. In unserer Institution werden die Ultraschalluntersuchungen immer von zwei Ärzten und einer Schwester an zwei Ultraschallgeräten parallel durchgeführt. Diese Konstellation findet in unserem Netzplan Berücksichtigung. Wir sehen bei unseren Arbeitsalgorithmen kaum Möglichkeiten, die Gesamtuntersuchungszeiten weiter zu verringern. Ein Ansatzpunkt wäre gegebenenfalls die Einführung einer computergestützten Spracherkennung zur schriftlichen Befundtexterstellung. Hiermit könnte eine geringe Zeitersparnis von eventuell 2 Minuten bezogen auf die Gesamtuntersuchungszeit erzielt werden. Bei der Einführung einer computergestützten Spracherkennung steht allerdings mehr die Entlastung des Arztes, der durch eine verbesserte Dokumentation nicht mehr als Schreibkraft 
fungiert, im Vordergrund als der reine Zeitgewinn. Die in der vorliegenden Studie verwendete Netzplantechnik ist ein effektives Instrument im Sinne des Qualitätsmanagements für Kliniken und Arztpraxen. Es lassen sich der Arbeitsablauf (=workflow) und die Maßnahmen, die im Laufe einer Behandlung oder Untersuchung ergriffen werden, analysieren. Erst auf der Grundlage von Netzplänen sollte eine Beschäftigungs- sowie Kostenplanung erfolgen. 


\subsection{Anwendung der Netzplantechnik für die Arbeitsprozessanalyse im klinischen Bereich}

\subsection{1 \\ Zielsetzung: Qualität und Effizienz im Routinebereich}

Die moderne Medizin zeichnet sich durch eine zunehmende Diversifizierung in diagnostische und therapeutische Funktionsbereiche mit einem hohen Grad an Spezialisierung aus. Im Rahmen eines Krankenhausaufenthalts durchläuft ein Patient zahlreiche dieser Funktionsbereiche. Für jeden einzelnen Patienten muss dabei eine hohe Ergebnisqualität erzielt werden. Das beinhaltet eine an der Fragestellung ausgerichtete, relevante diagnostische Aussage oder ein relevantes therapeutisches Ergebnis. Diese Vorgehensweise soll die Verfügbarkeit spezieller Einzelleistungen erhöhen, zu einer verbesserten Qualität der Einzelmaßnahme führen und die Kosten der Einzelmaßnahmen reduzieren. Der Aspekt der Kosteneinsparung gewinnt unter dem wachsenden Kostendruck im Gesundheitswesen besondere Bedeutung für Ärzte und Pflegepersonal. Er kann durch eine effiziente Arbeitsablaufsplanung positiv beeinflusst werden (73). Auch für den Patienten ergeben sich bei einer verbesserten Effizienz Vorteile in Form von verringerten Warte- und kürzeren Untersuchungszeiten, was die erlebte Prozessqualität steigert. Für den Funktionsbereich ergibt sich ein höherer Patientendurchsatz, was niedrigere Kosten pro Untersuchung erwarten lässt. Der vorliegende Beitrag stellt eine Methode vor, die auf Erhöhung der Effizienz standardisierter Abläufe bei gegebener Ergebnisqualität abzielt. Die Optimierung eines Arbeitsablaufes findet mit unterschiedlichen Maßnahmen auf verschiedenen Ebenen statt. Im Bereich des Projektmanagements, dem zahlreiche Ansätze für die Arbeitsablaufsplanung entlehnt sind, werden harte und weiche Maßnahmen unterschieden (81). Die weichen Maßnahmen betreffen den Faktor Mensch. Dazu zählen z.B. Konfliktmanagement sowie kommunikations- und motivationsfördernde Maßnahmen. Harte Maßnahmen zielen auf die Planung, Steuerung und Kontrolle aller formalen Aspekte eines Projekts. Zu den formalen Aspekten zählen z.B. der Informationsfluss, die personelle wie auch die Geräteausstattung und der Arbeitsablauf. Die im Folgenden vorgestellte Netzplantechnik (NPT), welche den harten Maßnahmen zuzuordnen ist, lässt sich im Zusammenhang mit weitgehend standardisierten Arbeitsabläufen verwenden, um diese im Hinblick auf Effizienz zu evaluieren. Im Rahmen einer Arbeitsablaufsanalyse werden für einen Arbeitsablauf Kenngrößen (Indikatoren) definiert, deren Veränderung als Maß für eine Verbesserung oder Verschlechterung des Ablaufs 
dient. Die Wahl der Kenngrößen richtet sich nach den Eigenschaften des Ablaufs. Für in hohem Maße standardisierte Abläufe, wie sie in Funktionsbereichen häufig anzutreffen sind und hier untersucht werden, zeichnet sich die Zeit als Kenngröße dadurch aus, dass sie metrisch skaliert und mit geringem Aufwand präzise messbar ist. In anderen Bereichen der klinischen Versorgung, in denen der Arbeitsablauf häufig spontan durch die Symptomkonstellation des Patienten bestimmt ist (z.B. auf einer Intensivstation), müssen andere Kenngrößen wie z.B. eine Bewertung des Informationsflusses oder der Mitarbeiterzufriedenheit im Zusammenhang mit anderen Evaluationsmethoden herangezogen werden

Die NPT wurde in den 50er Jahren im Rahmen der Planung und Überwachung größerer Projekte entwickelt (56). Mit ihr lassen sich auch Arbeitsablaufsstrukturen abbilden, planen und analysieren. So wird transparent, dass der Arbeitsablauf einer Ultraschalluntersuchung nicht nur durch die medizinische Maßnahme, sondern vor allem durch untersuchungsassoziierte administrative sowie vor- und nachbereitende Tätigkeiten bestimmt wird. Er umfasst bei näherer Betrachtung 10 Einzelarbeitsschritte, von denen die eigentliche Ultraschalluntersuchung nur einer ist (91;92). Wir haben die NPT im Funktionsbereich Sonographie in der diagnostischen Radiologie exemplarisch angewendet mit dem Ziel, den Arbeitsprozess in Hinblick auf Ressourceneinsatz und Kosten zu optimieren.

\subsubsection{Material und Methodik}

Als Vorläufer heutiger Netzplantechniken wurde das Gantt-Diagramm bereits im zweiten Weltkrieg entwickelt. Diese Technik ist auch heute noch im Einsatz, um zeitliche Zusammenhänge in Form eines Balkendiagramms zu visualisieren (81). Komplexe Ablaufstrukturen lassen sich hiermit jedoch nicht übersichtlich darstellen. Die wichtigsten Verfahren der Netzplantechnik sind die Critical Pathway Methode (CPM), Program Evaluation and Review Method (PERT) und Metra Potenial Method (MPM). Aus diesen fast gleichzeitig entstandenen Verfahren der NPT haben sich im Laufe der Zeit über 50 abgeleitete Verfahren mit spezifischen Besonderheiten entwickelt; jedes dieser Verfahren kann auch als ein Sonderfall einer übergeordneten Netzplantheorie betrachtet werden. Die Auswahl des Verfahrens richtet sich nach den Anforderungen des Projekts. Die CPM und PERT haben sich wegen ihrer Überschaubarkeit und universalen Anwendbarkeit für den Bereich der klinischen Medizin gegenüber anderen Netzplantechniken durchgesetzt. Mit ihnen lassen sich 
Struktur-, Zeit-, Kosten- und Beschäftigungs- bzw. Kapazitätsplanungen durchführen $(18 ; 49)$.

Unter einem Projekt wird ein abgrenzbares Vorhaben mit einer komplexen Realisierungsplanung (Projektplanung) verstanden. In der vorliegenden Arbeit repräsentiert die Ultraschalluntersuchung das Projekt. Bei der Projektplanung werden die einzelnen Realisierungsmaßnahmen näher betrachtet; diese entsprechen hier den einzelnen Arbeitsschritten der Untersuchung. Im Folgenden bezeichnen wir Arbeitsschritte synonym als Vorgänge oder Aktivitäten.

\subsubsection{Beschreibung des betrachteten Arbeitsplatzes}

Der beobachtete Arbeitsplatz ist Teil der radiologischen Klinik der Universitätsklinik Charité, Campus Virchow-Klinikum. Als Routine- und Volumenarbeitsplatz konzipiert, werden hier Patienten aus dem gesamten Klinikum mit verschiedensten Fragestellungen sonographisch untersucht. Dabei kommt die B-Bild-Untersuchung ebenso zum Einsatz wie die farbkodierte Dopplersonographie. Es stehen drei Sonographiegeräte in drei Untersuchungsräumen zur Verfügung. Ein viertes Gerät für die Versorgung von Patienten mit infektiösen Erkrankungen steht in einem weiteren Raum; diese Untersuchungen wurden hier nicht berücksichtigt. Die Untersuchungsbefunde werden von den Ärzten in Formularen an drei Apple Macintosh Computern erstellt und ausgedruckt. Dazu teilen sich drei Ärzte und ein Student im Praktischen Jahr einen Arbeitsraum mit 2 medizinisch-technischen Röntgenassistenten (MTRA) und einem Zivildienstleistenden, die dort administrative Aufgaben wahrnehmen.

Die Administration mit Zeitplanung und Abrechnung erfolgt computergestützt über das klinikinterne RIS (Radiologisches Organisations- und Befundsystem), wofür zwei Terminals zur Verfügung stehen. Befunde und Bilder werden konventionell archiviert. Zwei Telefone stehen zur Verfügung, von denen in der Regel eines von den MTRA für Terminabsprachen, das andere für Telefonate seitens der Ärzte benutzt wird. Der Arbeitsplatz ist als Ausbildungsplatz konzipiert. Laut Plan sind ihm ein Oberarzt mit langjähriger Ultraschallerfahrung, ein Assistenzarzt mit bis zu einjährigerErfahrung und ein Anfänger, in der Regel ein Arzt im Praktikum, zugeteilt. Zusätzlich ist regelmäßig ein Medizinstudent im Praktischen Jahr anwesend. Zum Zeitpunkt der Untersuchung hatten der Oberarzt ca. 40.000 Untersuchungen, der 
Assistenzarzt ca. 1200 Untersuchungen und der Anfänger ca. 200 Untersuchungen durchgeführt.

Pro Tag werden im Routinebereich durchschnittlich 33 [Range 9-55] Untersuchungen an 27 [Range 9-46] Patienten durchgeführt.

\subsubsection{Benchmarking von Arbeitsprozessen}

Im klinischen Bereich können aus prozesstechnischer Sicht zwei Arten von Arbeitsprozessen unterschieden werden. Arbeitsprozesse mit vorhersagbarem, weil in erster Linie durch die Ausführenden determiniertem Ablauf finden in den Funktionsbereichen statt, wo in großer Zahl spezielle und wohldefinierte therapeutische und diagnostische Maßnahmen durchgeführt werden. Im Stationsbereich, speziell in der Intensivbehandlung, werden Abläufe stärker durch das Symptombild des Patienten bestimmt und sind von den Ausführenden nur eingeschränkt oder auf einer übergeordneten, unscharfen Ebene planbar. Prozessqualität kann über unterschiedliche Parameter (Indikatoren, Kenngrößen) erfasst werde. Die Wahl der Parameter ist von der Art des Prozesses sowie von der Sichtweise des Betrachters abhängig: Was erachtet er als entscheidend für die Beurteilung von Prozessqualität? Neben metrisch erfassbaren Größen wie der Zeit lassen sich auch subjektive Bewertungen heranziehen wie z.B. die Kunden- oder Mitarbeiterzufriedenheit. Die Erfassung von Zeit macht nur in Bereichen Sinn, deren Abläufe in hohem Maße standardisiert und damit auf dieser Ebene vergleichbar sind. In dieser Arbeit wird ein Arbeitsablauf aus einem diagnostischen Funktionsbereich betrachtet. Eine Gruppe von Spezialisten führt wiederholt eine eng definierte Menge von Vorgängen in einer bestimmten Reihenfolge aus. Für einen solchen Ablauf lässt sich die Zeit als leicht zu erfassende metrische Größe zur Beurteilung der Prozessqualität heranziehen. Beide hier verwendeten Verfahren bedienen sich der Zeit als Kenngröße zur Prozessbeurteilung.

\subsubsection{Einführung in die Netzplantechnik}

Die Netzplantechnik wurde als Werkzeug der Projektplanung im Rahmen des Projektmanagements entwickelt. Sie ist somit eine Teildisziplin im weiten Feld des Projektmanagements. Im Folgenden wird der Kontext des Projektmanagements beschrieben, in den die Netzplantechnik eingebettet ist. Projektplanung ist eine Teildisziplin des Projektmanagements. Sie umfasst die - 
soweit möglich - gedankliche Vorwegnahme aller Aktivitäten, die im Verlauf eines Projekts durchzuführen sind (81). Um im Rahmen komplexer Projekte die Übersicht zu wahren, wurden Werkzeuge zur Visualisierung sowie Kalkulation der projektbestimmenden Größen Zeit und Kosten entwickelt. Die Netzplantechnik als zentrales Werkzeug für diese Aufgabe wird im Folgenden vorgestellt.

\subsubsection{Definition eines Netzplans}

Die im Folgenden beschriebenen Verfahren werden unter der Bezeichnung Netzplantechnik zusammengefasst. Diese ist laut DIN 69900 definiert als „alle Verfahren zur Analyse, Beschreibung, Planung, Steuerung, Überwachung von Abläufen auf der Grundlage der Graphentheorie, wobei Zeit, Kosten, Einsatzmittel und weitere Einflussfaktoren berücksichtigt werden können“ Mathematisch ist ein Netzplan eine spezielle Form eines Graphen. Unter einem Graphen versteht man eine (endliche oder unendliche) Menge von Knoten, die durch eine (endliche oder unendliche) Menge von Kanten einander zugeordnet sind (81). Dieser kann zeichnerisch dargestellt werden, indem z.B. die Knoten als Kreise und die Kanten als Verbindungslinien gezeichnet werden. Sind alle Knoten über Kanten dergestalt miteinander verbunden, dass jeder Knoten von jedem anderen Knoten über eine oder mehrere Kanten erreicht werden kann, spricht man von einem zusammenhängenden Graphen. Kanten können mit einer Richtung versehen werden, sie heißen dann Pfeile. Sind alle Kanten in einem Graphen Pfeile, spricht man von einem gerichteten Graphen. Ein Pfeil, der einen Knoten mit sich selbst verbindet, heißt Schleife. Kanten können auch Werte zugeordnet werden, welche die Kanten näher spezifizieren. Sind allen Kanten Werte zugeordnet, spricht man von einem bewerteten

Graphen.

Definition: Ein endlicher, zusammenhängender, bewerteter und gerichteter Graph ohne Schleifen heißt Netzplan (81). Nicht alle Varianten der Netzplantechnik verwenden Netzpläne im mathematischen Sinne.

\subsubsection{Aktivitäten/Vorgänge}

Aktivitäten oder Vorgänge (die Begriffe werden synonym verwendet) beschreiben die einzelnen Arbeits-, Projekt- oder Prozessschritte, die zum Erreichen von Zielen durchgeführt werden müssen. Im Netzplan sind sie charakterisiert durch. 
1. ihre Position im Projektgeschehen mit der Verknüpfung zu anderen Vorgängen

2. ihre Auftretenswahrscheinlichkeit,

3. ihre Dauer und

4. die zur Durchführung nötigen Ressourcen.

Die Position der Vorgänge wird über ihre Anordnungsbeziehung beschrieben (s.u.). Die Auftretenswahrscheinlichkeit $\mathrm{p}_{\mathrm{A}}$ beschreibt die Wahrscheinlichkeit, mit der eine Aktivität in einem Projekt tatsächlich durchgeführt wird. In Arbeitsabläufen kommt nicht jede Aktivität regelmäßig in jedem Zyklus oder immer an der gleichen Stelle vor. Je nach Auftretenswahrscheinlichkeit und Position eines Vorgangs im Arbeitsablauf lassen sich vier Typen unterscheiden. Für diese Arbeit werden die vier Typen den Bezeichnungen $\mathrm{la}$, Ib, Ila und Ilb zugeordnet. Die römische Ziffer steht für die Position des Vorgangs im Arbeitsablauf (I: feste Position; II: variable Position), der Buchstabe für die Auftretenshäufigkeit (a: regelmäßiges Auftreten $\left(p_{A}=1\right) ; b$ : unregelmäßiges Auftreten $\left(p_{A}<1\right.$ oder $\left.\left.p_{A}>1\right)\right)$.

1. Typ la: Der Vorgang findet in jedem Zyklus genau einmal an einer festgelegten Stelle im Arbeitsablauf statt.

2. Typ Ib: Der Vorgang wird nicht regelmäßig durchgeführt, ist jedoch an einer festgelegten Stelle im Arbeitsablauf positioniert. So wird z.B. beim fortgeschrittenen Untersucher nicht jede Untersuchung vom Oberarzt nachbefundet; wenn er das jedoch tut, geschieht das immer unmittelbar nachdem der Untersucher seine Untersuchung abgeschlossen hat.

3. Typ Ila: Der Vorgang wird genau einmal pro Zyklus durchgeführt, kann jedoch an einer beliebigen Stelle im Arbeitsablauf oder sogar zeitlich getrennt vom eigentlichen Arbeitsablauf durchgeführt werden. Dies gilt für viele administrative Tätigkeiten wie Anmeldung, Bilder in Archivtüten sortieren etc., die dann ausgeführt werden, wenn gerade nichts anderes zu tun ist.

4. Typ Ilb: Der Vorgang findet unregelmäßig statt und kann irgendwo im Arbeitsablauf auftreten. Dies ist z.B. beim Abholen der Filme vom Drucker der Fall $\left(p_{A}<1\right.$, da die Filme für mehrere Untersuchungen zusammen geholt werden) oder bei der Anmeldung von Patienten $\left(p_{A}>1\right.$, da einige Patienten abgemeldet und für diese wieder neue angemeldet werden, um auf die gewünschte Patientenzahl zu kommen).

Die Dauer kann entweder deterministisch oder stochastisch sein. Deterministische Dauern sind von vornherein bekannt und festgelegt. Mit stochastischen Mitteln werden Vorgangsdauern beschrieben, die im Vorfeld nur abgeschätzt werden 
können. Dabei wird ein Zeitraum angegeben, innerhalb dessen die Dauer des Vorgangs mit einer gegebenen Wahrscheinlichkeit liegt.

Die Ressourcen können Arbeitskraft und Material sein.

\subsection{8 .Ressourcenplanung}

Ereignisse sind Strukturelemente ohne Dauer. So hat z.B. jeder Vorgang ein Anfangs- und ein Endereignis; jedes Projekt beginnt mit einem Startereignis und endet mit dem Zielereignis. Teilziele können als Ereignisse im Netzplan modelliert werden. Ereignisse, denen im Projektverlauf besondere Bedeutung zukommt, heißen Meilensteine. Ereignisse sind charakterisiert durch ihre Position im Projektgeschehen.

\subsubsection{Anordnungsbeziehungen}

Sind die einzelnen Vorgänge und Ereignisse definiert, werden ihre Abhängigkeiten über Anordnungsbeziehungen festgelegt. Im ersten Ansatz wird man sich die Abfolge der Aktivitäten und Ereignisse aus den Vorgaben logischer bzw. technischer Zusammenhänge herleiten:

1. Welcher Vorgang muss begonnen oder abgeschlossen oder welches Ereignis muss eingetreten sein, damit dieser Vorgang durchgeführt werden kann?

2. Diese Frage bestimmt den Vorgänger der aktuell betrachteten Aktivität bzw. des aktuell betrachteten Ereignisses.

3. Welcher Vorgang kann im Anschluss an den Beginn oder das Ende dieser Aktivität durchgeführt werden? Diese Frage bestimmt den Nachfolger der aktuell betrachteten Aktivität bzw. des aktuell betrachteten Ereignisses.

4. Je nach Art der Abhängigkeit unter den Vorgängen sind verschiedene Typen von Anordnungsbeziehungen definiert. Nicht alle lassen sich in jeder Netzplantechnik modellieren.

5. Die Ende-Anfang (EA) Beziehung wird am häufigsten verwendet. Sie beschreibt die Beziehung zwischen dem Ende des einen und dem Beginn eines anderen Vorgangs. Nach DIN 69900 heißt diese Abhängigkeit Normalfolge (NF).

6. Die Anfang-Anfang Beziehung (AA-Beziehung, DIN 69900: Anfangsfolge $(\mathrm{AF})$ ) beschreibt eine Beziehung zwischen dem Beginn zweier Vorgänge.

7. Die Anfang-Ende Beziehung (AE-Beziehung, DIN 69900: Sprungfolge (SF)) beschreibt die Beziehung zwischen dem Beginn des einen und Ende eines anderen Vorgangs. 
8. Die Ende-Ende Beziehung (EE-Beziehung, DIN 69900: Endfolge (EF)) beschreibt eine Beziehung zwischen dem Ende zweier Vorgänge.

Zusätzlich kann ein zeitlicher Abstand Z zwischen den Beziehungspunkten definiert werden. Dieser Abstand kann kleiner, größer oder gleich Null sein. Es können Grenzwerte für den Abstand definiert werden: Der Zeitabstand, der den frühesten Zeitpunkt für den Beginn oder, je nach Anordnungsbeziehung, das Ende eines Vorgangs modelliert, wird mit MINZ, der Zeitabstand für den spätesten Zeitpunkt mit MAXZ bezeichnet. Die jeweiligen Bedingungen, die damit modelliert werden können, heißen MAX bzw. MIN plus Kürzel der Anordnungsbeziehung (z.B. MAXEA, MINAA). Beispiel: Wenn ein Vorgang drei Tage vor Ende seines Vorgängers oder später beginnen kann, liegt hier eine MINEA-Bedingung vor mit MINZ=-3t.

\subsubsection{Darstellungsformen in der Netzplantechnik}

Wahlweise können nur Vorgänge, nur Ereignisse oder eine Kombination von Vorgängen und Ereignissen dargestellt werden. Diese können als Pfeile oder Knoten innerhalb eines Netzplans auftreten. Daraus ergeben sich verschiedenen Möglichkeiten der Darstellung eines Projektablaufs.

\subsubsection{Vorgangsorientierter Netzplan}

Hier werden bei der Planung nur Vorgänge betrachtet. Ereignisse werden nicht dargestellt. Die Beschreibung der Vorgänge und deren Reihenfolge kann auf zwei Arten erfolgen: Vorgangspfeilnetz (Abbildung 4-4): Hier werden die Vorgänge als beschriftete Pfeile dargestellt, die über Knoten miteinander verbunden sind. Die Knoten werden üblicherweise als Kreise gezeichnet.

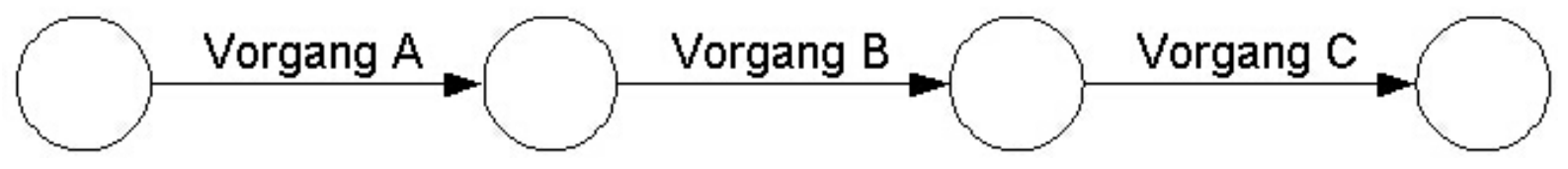

Abbildung 4-4: Vorgangspfeilnetz 


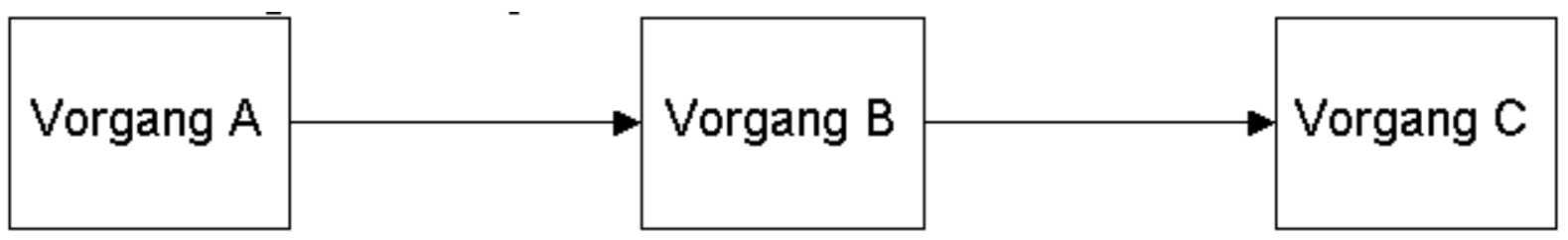

Abbildung 4-5: Vorgangsknotennetz

Ereignisorientierter Netzplan: Bei einem ereignisorientierten Netzplan liegt der Fokus der Planung und Darstellung des Projektablaufs auf den Ereignissen. Da es nicht üblich ist, Ereignisse als Pfeile darzustellen, ergibt sich nur die Darstellungsform. Ereignisknotennetz (Abbildung 4-6): Hier werden die Ereignisse als beschriftete Knoten dargestellt. Sie werden durch Pfeile so verknüpft, wie es ihrer Reihenfolge im Projektablauf entspricht. Aufgrund einer Reihe von Vorteilen gegenüber den anfangs favorisierten Vorgangspfeilnetzen ist dies heute die übliche Darstellungsart (81).

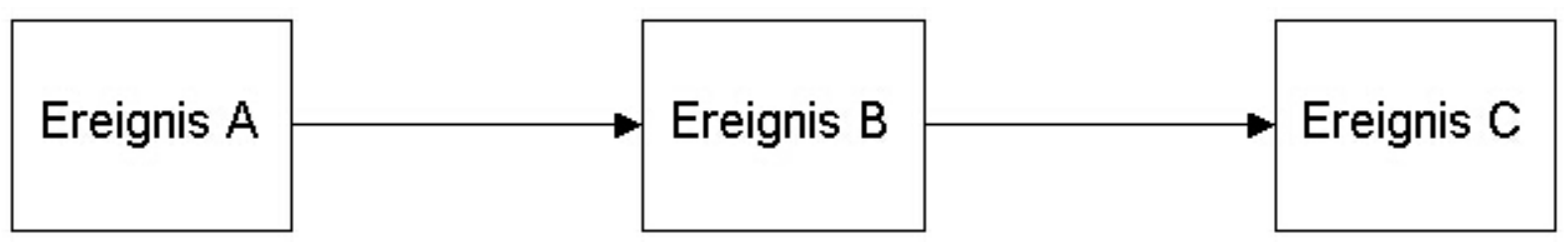

Abbildung 4-6: Ereignisknotennetz

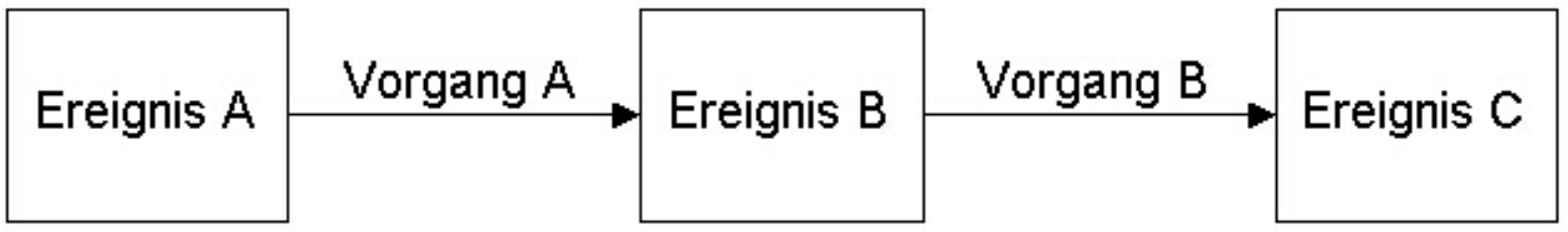

Abbildung 4-7: Gemischtorientierter Netzplan

\subsubsection{Varianten der Netzplantechnik}

Alle unten aufgeführten Varianten der Netzplantechnik sind letztlich Spezialfälle eines allgemeinen Ablaufmodells der Netzplantechnik, in dem alle Ablaufelemente und alle Abhängigkeiten berücksichtigt werden und bei dem die vollständige Ablaufstruktur aus dem Netzplan ersichtlich ist. Es gibt nicht verschiedene Netzplantechniken, sondern nur Varianten eines allgemeinen Modells, die für Spezialfälle anwendbar sind. 


\section{Ein Vorläufer: Gantt-Diagramm}

Ein Vorläufer heute üblicher Netzplantechniken wurde erstmals von Henry L. Gantt zur Planung einer Produktionsstraße im ersten Weltkrieg entwickelt (GanttDiagramm). Es handelt sich um ein formales Instrument für deterministische Projektabläufe. Die einzelnen Vorgänge wurden als Balken mit einer Länge entsprechend ihrer Dauer aufgezeichnet. Anhand ihrer Reihenfolge konnte abgeleitet werden, welche Vorgänge wann beginnen würden. So zeigt der Balkenplan (in seiner Grundform; Varianten wurden entwickelt) nur die zeitliche Anordnung von Vorgängen, nicht aber deren logische Abhängigkeiten. Diese Darstellungsform ist heute noch im Einsatz, wenn es um die Visualisierung der Termin- und Ressourcenplanung und um die Präsentation des Projektablaufs geht; auch in dieser Arbeit wird sie verwendet, um zeitliche Zusammenhänge zwischen Vorgängen zu verdeutlichen. Nachteile entstehen, wenn die Steuerung und Simulation komplexer Projekte mit den Abhängigkeiten der einzelnen Vorgänge visualisiert werden sollen. Hier wird der Balkenplan aufgrund seines Umfangs zu unübersichtlich und ist daher nur für die Grobplanung und die Personal- und Einsatzmittelplanung anzuwenden.

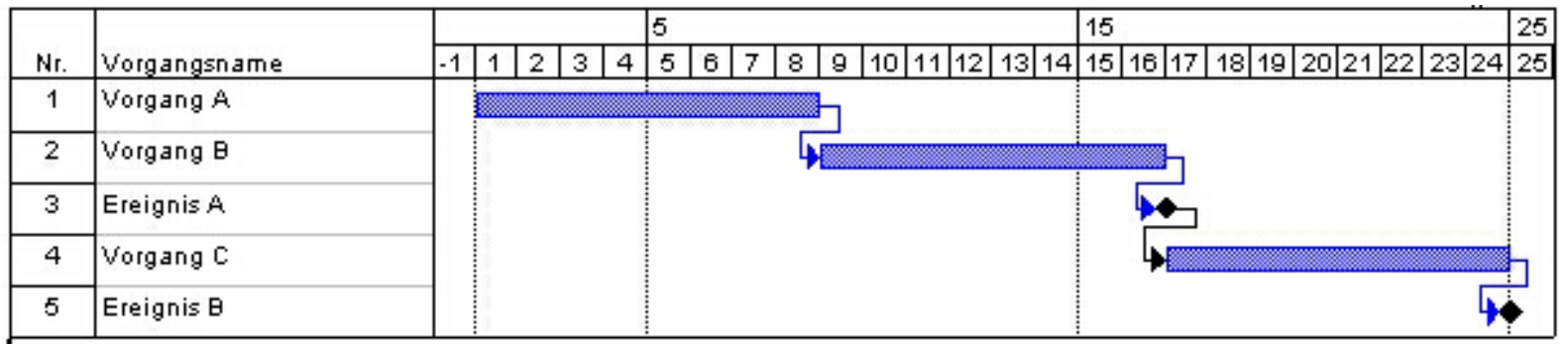

Abbildung 4-8: Gantt-Diagramm

Aus diesem Grund wurden Mitte der 50er Jahre fast zeitgleich neuere Verfahren in den USA und Frankreich entwickelt. Alle Verfahren unterscheiden zwischen Vorgängen (den eigentlichen Aktivitäten mit einer bestimmten Dauer) und Ereignissen (definierte Zeitpunkte oder Ergebnisse ohne Dauer, z.B. das Erreichen einer Zielsetzung oder das Ende eines Vorgangs). Grundsätzlich werden zwei Verfahren der Netzplantechnik unterschieden. Verfahren für deterministische Projektabläufe kommen zum Einsatz, wo die einzelnen Vorgänge mit ihrer Dauer und Anordnung vorhersagbar sind (CPM, MPM, PERT). Bei stochastischen Projektabläufen sind die einzelnen Vorgänge zum Zeitpunkt der Planung noch nicht vorhersagbar, wie das z.B. bei komplexen Entwicklungs- oder 
Forschungsprojekten der Fall ist. Hier werden andere Verfahren verwendet (GERT, GAN).

\subsubsection{Verfahren für deterministische Projektabläufe}

In deterministischen Projektabläufen sind alle vorkommenden Aktivitäten mit ihrer Dauer bekannt. Anordnungsbeziehungen können nur über UND logisch verknüpft sein. Es sind keine Wiederholungen und keine Ausführungsbedingungen vorgesehen; alle Vorgänge des Projekts werden genau einmal durchlaufen. Ein Feedback (Reaktion auf nicht vorhersehbare Ereignisse im Projekt) ist nicht vorgesehen.Critical Pathway Method (CPM): CPM wurde 1956 vom Chemieunternehmen DuPont entwickelt, um komplexe Bauprojekte zu managen. Vorgänge werden mit Hilfe von Pfeilen visualisiert, die über Ereignisknoten miteinander verbunden sind. CPM entspricht damit einem (gemischtorientierten) Vorgangspfeilnetz. Scheinvorgänge wurden eingeführt, um Abhängigkeiten zwischen Ereignissen darzustellen, die nicht durch reale Vorgänge verknüpft sind.

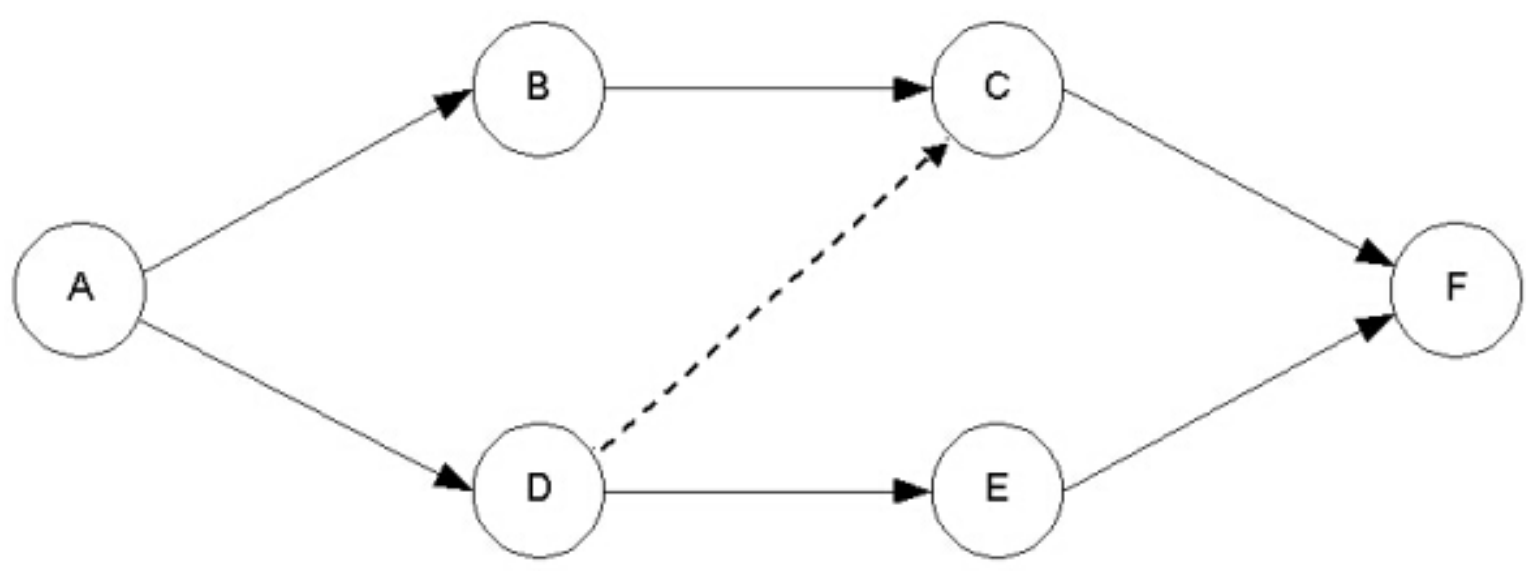

Abbildung 4-9: Schematische Darstellung eines Netzplans mit Scheinvorgang (gestrichelte Linie)

Program Evaluation and Review Technique (PERT): PERT wurde 1957 von der USamerikanischen Navy im Rahmen der Entwicklung der Polaris-Rakete erfunden. Mit beachtlichem Erfolg: Gegenüber der erwarteten Projektdauer wurden zwei Jahre Entwicklungszeit eingespart. Auch PERT stellt Ereignisse als Knoten dar, die durch Pfeile miteinander verbunden sind (Ereignisknotennetz). Diese Pfeile entsprechen 
jedoch keinen Vorgängen; Information über Vorgänge ist in dieser Darstellungsform nicht

enthalten.

PERT enthält bereits ein stochastisches Element in Form von unscharfen Vorgangsdauern. Dieses Element wird für die Berechnung von optimistischen, realistischen und pessimistischen Zeiten herangezogen: Für jeden Vorgang wird die optimistische, die realistische und die pessimistische Zeit ermittelt. Die optimistische Zeit entspricht der kürzesten gemessenen Zeit, die pessimistische der längsten gemessenen Zeit. Die realistische Zeit errechnet sich aus dem Mittelwert aller Messwerte, der pessimistischen und der optimistischen Zeit. Der Mittelwert wird dabei viermal so hoch gewichtet wie die beiden anderen Werte, sodass sich die folgende Formel ergibt:

$t_{\text {real }}=\frac{t_{o p t}+4 * \bar{t}+t_{\text {pess }}}{6}$

Die Verteilung der Gewichtung wurde von den Entwicklern der Methode willkürlich festgelegt.

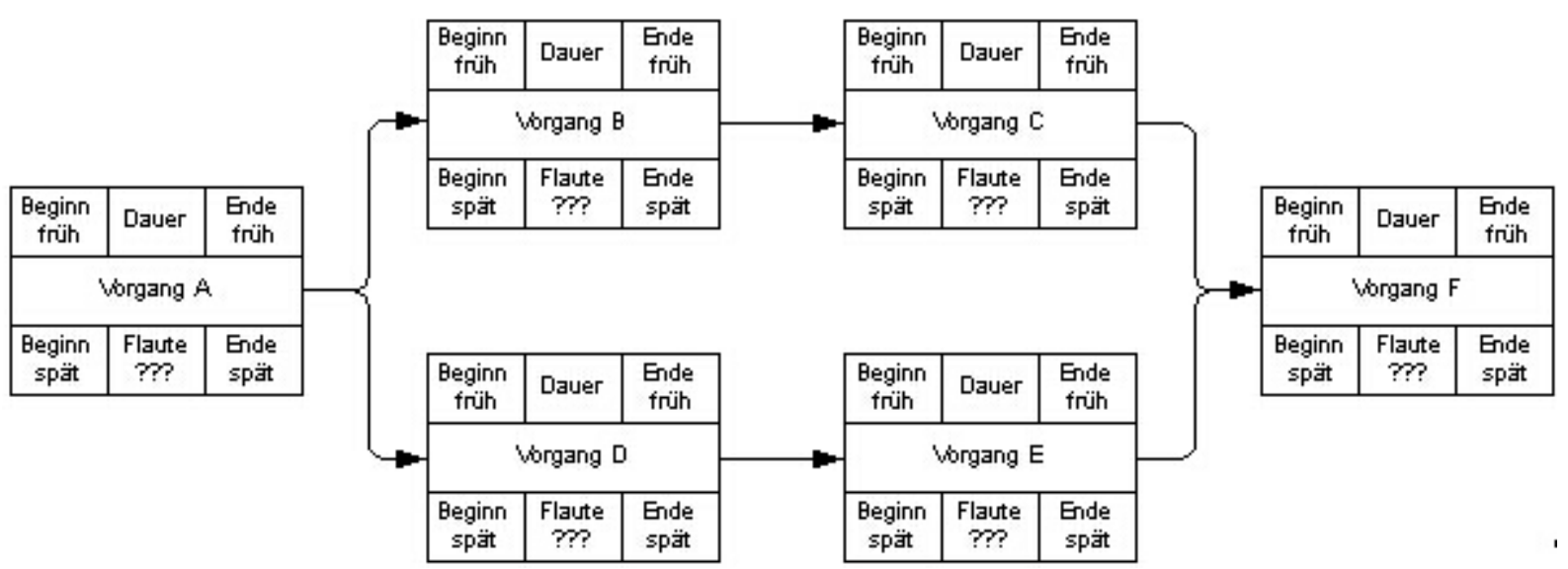

Abbildung 4-10: Metra-Potential-Skizze

\subsubsection{Metra Potential Method}

Metra Potential Method (MPM): Die Metra Potential Method entstand 1957 in Frankreich. Sie verwendete als Erste Vorgangsknotennetze: Vorgänge werden als Knoten dargestellt, die zur Darstellung ihrer Abhängigkeiten über Pfeile miteinander verbunden sind. 


\subsubsection{Verfahren für stochastische Projektabläufe}

Viele Projekte, speziell im Bereich Forschung und Entwicklung, genügen nicht den Anforderungen, die für die Planung mit deterministisch ausgerichteten Netzplantechniken gelten. Insbesondere drei Einschränkungen wurden identifiziert:

1. In vielen Projekten werden einige Vorgänge nicht mit Sicherheit ausgeführt, sondern nur mit einer bestimmten Wahrscheinlichkeit in Abhängigkeit vom Projektverlauf.

2. Der Beginn eines Vorgangs kann von mehreren anderen Vorgängen abhängig sein in der Art, dass die Ausführung einer Teilmenge der vorausgehenden Vorgänge als Voraussetzung genügt. Diese Art von logischer Verknüpfung („ODER“) ist in deterministischen Projektabläufen ebenfalls nicht vorgesehen.

3. Im Verlauf eines Projekts kann es nötig sein, einen oder mehrere Vorgänge wiederholt durchzuführen, bis ein gewünschtes Ziel erreicht ist.

Aus diesem Grund wurden die vorhandenen Techniken um Auftretenswahrscheinlichkeiten von Vorgängen und Beziehungen, Schleifen (Wiederholungen), logische Verknüpfungen und Bedingungen erweitert. Die erweiterten Netzplanmodelle nähern sich einem allgemeinen Netzplanmodell an. Von der Perspektive des erweiterten Netzplanmodells aus gesehen, können deterministische Projektabläufe als Spezialfälle von stochastischen Projektabläufen betrachtet

werden.

Mitte der 60er Jahre machte Eisner einen ersten Schritt in der Erweiterung deterministischer Projektmodelle durch die Einführung von Entscheidungskriterien für Vorgänge (sogenannte Knotenausgänge). Seine Ideen wurden aufgegriffen und in eigenständige Netzplantechniken eingebaut. Generalized Activity Networks (GAN): GAN wurde von Elmaghraby entwickelt (66). Er führte neben der Berücksichtigung stochastischer Vorgangsdauern, wie sie bereits aus PERT bekannt waren, und den Knotenausgängen von Eisner verschiedene Knoteneingänge als logische Verknüpfungen Graphical Evaluation and Review Technique (GERT): GERT wurde etwa zeitgleich zu GAN von Pritsker und Happ entwickelt (101). Analog zu Elmaghraby führten sie verschiedene Knoteneingänge als logische Verknüpfungen ein. Außerdem fügten sie zur stochastischen Vorgangsdauer auch eine Ausführungswahrscheinlichkeit hinzu. 


\subsubsection{Vergleich der Verfahren}

Tabelle 4-2: Netzplantechniken im Vergleich. (IOR: Inclusive OR. IOR ist WAHR, wenn aus einer Menge von Bedingungen eine oder mehrere erfüllt sind. EOR: Exclusive OR. EOR ist WAHR, wenn aus einer Menge von Bedingungen genau eine erfüllt ist.)

\begin{tabular}{|c|c|c|c|c|c|c|c|c|c|c|c|}
\hline \multirow{3}{*}{$\begin{array}{l}\text { Netzplan } \\
\text { technik }\end{array}$} & \multirow[t]{3}{*}{ Darstellung } & \multicolumn{5}{|c|}{ Vorgänge } & \multirow[t]{3}{*}{ Ereignisse } & \multirow{2}{*}{\multicolumn{3}{|c|}{$\begin{array}{l}\text { Logische } \\
\text { Operanden }\end{array}$}} & \multirow{3}{*}{$\begin{array}{l}\text { Besonderh } \\
\text { eiten }\end{array}$} \\
\hline & & \multicolumn{3}{|l|}{ Auftreten } & \multicolumn{2}{|c|}{ Vorgangsdauer } & & & & & \\
\hline & & $\begin{array}{l}\text { Determin } \\
\text { istisch }\end{array}$ & $\begin{array}{l}\text { stocha- } \\
\text { stisch }\end{array}$ & $\begin{array}{l}\text { Wahrsch } \\
\text { einlichkei } \\
t\end{array}$ & $\begin{array}{l}\text { Determi } \\
\text { nistisch }\end{array}$ & $\begin{array}{l}\text { stocha- } \\
\text { stisch }\end{array}$ & & UND & IOR & EOR & \\
\hline CPM & Ereignisknoten & $\bullet$ & & & $\bullet$ & & $\bullet$ & $\bullet$ & & & $\begin{array}{l}\text { Scheinvorg } \\
\text { änge }\end{array}$ \\
\hline PERT & Ereignisknoten & • & & & & - & • & • & & & \\
\hline MPM & Vorgangsknoten & - & & & - & & & • & & & \\
\hline GERT & Vorgangsknoten & & - & • & & - & & • & • & - & \\
\hline GAN & Vorgangsknoten & & - & & & - & & • & - & - & \\
\hline
\end{tabular}

\subsubsection{Ressourcenplanung}

Seinen Wert für die Projektplanung und -überwachung erhält ein Netzplan durch die Möglichkeit, den Ressourceneinsatz zu planen, da Ressourcen den Projektverlauf und die zu erwartenden Kosten bestimmen. Ressourcen können Arbeitskraft oder Material sein; ihre Zuteilung erfolgt in den Einheiten [Arbeitskraft $x$ Zeit] bzw. [Material $x$ Zeit]. Jedem Vorgang werden die Ressourcen zugeteilt, die für seine Durchführung nötig sind. Bei Vorgängen, die eine freie Gestaltung der Menge einer zugeteilten Ressource erlauben, hat dies den Vorteil, dass später die Dauer eines Vorgangs flexibel über die zugeteilte Ressourcenmenge reguliert werden kann. In der vorliegenden Arbeit waren alle ermittelten Vorgänge an genau eine Arbeitskraft gebunden: Jeder Vorgang wurde im gesamten Verlauf von einer Arbeitskraft durchgeführt, somit waren die Dauern der Einzelvorgänge nicht ressourcengesteuert. Durch die Integration von Ressourcen in einen Netzplan werden drei Dinge ermöglicht. Erstens ist eine genaue Ressourcenplanung in Abhängigkeit von den durchzuführenden Aktivitäten möglich. Dies erlaubt zweitens eine genaue Kostenplanung. Drittens wird die logische durch die ressourcengesteuerte Ablaufplanung ergänzt, sodass beispielsweise Engpässe an Arbeitskraft oder Material und damit Verzögerungen vorausschauend vermieden werden können. 
Auch können zeitkritische Vorgänge verkürzt werden durch die Zuteilung zusätzlicher Ressourcen.

Von den Materialressourcen finden nur diejenigen Eingang in die Kapazitätsberechnungen, deren Veränderung einen erwarteten Einfluss auf den Projektablauf haben. Einige Materialressourcen wie z.B. der Befundungs- und Organisationsraum wurden gleichbleibend von allen beteiligten Arbeitskräften genutzt und in die Kapazitätsplanung nicht aufgenommen. Sie sind in der Beschreibung des Arbeitsplatzes aufgeführt und finden in das Projektmodell insofern Eingang als sie die Voraussetzung sind, um den Arbeitsprozess in der hier dargestellten Weise durchführen zu können.

Beim Material unterscheidet man Verbrauchs- und Gebrauchsmaterial. Beide Arten verursachen Kosten: Verbrauchsmaterial muss jeweils neu beschafft werden, um eine Aktivität durchführen zu können. Es wird einem Vorgang in festen Mengen zugeteilt und beeinflusst die Kostenplanung, nicht jedoch die Zeitplanung. Gebrauchsmaterial verursacht Anschaffungs-, Abschreibungs- und Wartungskosten und wird einem Vorgang in Material mal Zeiteinheit zugeteilt. In der Kapazitätsberechnung beeinflusst es die Kosten- und Zeitplanung und verhält sich damit analog zur Arbeitskraft.

\subsubsection{Software}

Da der Prozess der Erstellung eines Netzplans bis hin zu einer optimalen Anordnung der Aktivitäten unter Berücksichtigung der Ressourcen komplex ist, bedarf es geeigneter Werkzeuge zur Durchführung. In der vorliegenden Arbeit wurde das Programm „Project 2000“ der Firma Microsoft verwendet, welches sowohl unscharfe Vorgangsdauern nach PERT wie auch die Analysemethode des Kritischen Pfades beherrscht.

\subsubsection{Anfertigung eines Netzplans}

Ein Netzplan wird in vier Phasen erstellt.

\subsubsection{Phase I: Strukturermittlung}

Vorgänge und Ereignisse ermitteln: Zur Definition der Ereignisse wurden die einzelnen Arbeitsschritte, die zur Durchführung einer Ultraschalluntersuchung notwendig sind, durch zwei nicht in den Arbeitsablauf eingebundene Beobachter (Ärzte) ermittelt. Sie begleiteten je 10 Untersuchungen und notierten alle identifizierten Aktivitäten in einer Vorgangsliste.

Die einzelnen Vorgänge und Ereignisse wurden in Stufen ermittelt. Ausgehend von 
einem groben Übersichtsplan, der übergreifende Arbeitsschritte darstellt, wurden unter Mitwirkung der MTRA und Ärzte feinere Strukturen innerhalb der übergreifenden Abschnitte definiert. Die Abschnitte wurden aufgabenorientiert und nicht ressourcenorientiert definiert; Ressourcen wurden den einzelnen Arbeitsschritten erst zugeordnet, nachdem die Vorgänge in hinreichender Auflösung festgelegt waren.

Ressourcen zuteilen:

Jedem Vorgang werden die Ressourcen, die zur Durchführung nötig sind, zugeordnet. Bei der Protokollierung der Vorgänge wird bereits miterfasst, welche Person den Vorgang durchführt und welche Materialien dafür notwendig sind. Die einzelnen Mitarbeiter werden in Abhängigkeit von ihren Kompetenzen Ressourcengruppen zugeteilt. In der Projektplanung kann dann jedem Vorgang ein oder mehrere Mitarbeiter zugeteilt werden, dessen Kompetenzprofile mit dem Anforderungsprofil der Aktivität übereinstimmt. In der vorliegenden Arbeit werden die Ressourcengruppen Ärzte und MTRA unterschieden.

Anordnungsbeziehungen definieren (logisch/technisch):

Die Vorgänge werden in logische Beziehung zueinander gesetzt. Zusätzlich wird ein Startereignis als Quelle und ein Endereignis als Senke definiert. Alle Vorgänge müssen zwischen diesen beiden Ereignissen liegen. Für jeden Vorgang wird ermittelt, welche Vorgänge unmittelbar vor der Durchführung ausgeführt sein müssen und welche daran anschließend. Dabei werden in diesem Schritt sowohl logische Zusammenhänge als auch vorher festgelegte Regeln für die Durchführung einer Untersuchung berücksichtigt. So ist z.B. eine Regel, dass ein neuer Patient erst aufgelegt werden darf, wenn der Untersuchungsraum gereinigt ist. Ein logischer Zusammenhang ist z.B., dass die Untersuchung durch den Arzt erst beginnen kann, wenn der Patient auf der Untersuchungsliege liegt. Es können mehrere parallele unabhängige Vorgangsstränge existieren, die allein über Quelle und Senke zusammenhängen. Ein Strang aus Typ-la-Vorgängen (der auch Typ-lb-Vorgänge enthalten kann) wird dann als Hauptstrang definiert. Hier ist das z.B. bei den Strängen „Untersuchung“ und „Anmeldung“ der Fall. Zu jeder Untersuchung gehört eine Anmeldung. Der Vorgang der Anmeldung findet jedoch in praxi mehrere Tage vor der Untersuchung statt, wobei im Arbeitsablauf ständig Anmeldungen und Untersuchungen parallel laufen. Die kausale Pause zwischen Anmeldung und Untersuchung im Netzplan zu berücksichtigen, würde zu einer 
erheblichen Verzerrung des ermittelten Aufwandes für eine Untersuchung führen. Also bildet man die beiden Tätigkeiten parallel ab und kommt so zu einer realistischen Aufwandsabschätzung. Nachdem so ihre Reihenfolge festgelegt war, wurde für eine bessere Übersicht jedem Vorgang ein Buchstabe zugeordnet.

\subsubsection{Phase II: Messdatenerfassung}

Vorgangsdauern:

Die zur Berechnung verwendeten Vorgangsdauern und Ressourcenmengen sind entweder Erfahrungswerte, Schätzwerte oder, wie in diesem Fall, vorab in praxi ermittelte und statistisch aufbereitete Daten. Um den Messvorgang effizient zu gestalten, wurde eine Software erstellt. Als Werkzeuge kamen dabei die Datenbank Microsoft Access 2000 und die Programmiersprache Microsoft Visual Basic for Applications zum Einsatz. Die Kernfunktionalität der Software ist die Automatisierung und Verwaltung mehrerer parallel ablaufender Messungen von Vorgängen. Beim Start der Messung wird die durchführende Ressource und die Aktivität ausgewählt. Laufende Messungen werden in einer Auswahlliste angezeigt. $\mathrm{Zu}$ jeder Messung kann zusätzliche Information zur Protokollierung der Rahmenbedingungen erfasst werden, wie z.B. die Untersuchungsart oder die Transportart des Patienten. Wird die Ausführung eines Vorgangs unterbrochen, z.B. wenn ein Telefonat beantwortet werden muss, kann die Messung in der Software auf Pause gesetzt und anschließend fortgeführt werden. Die Software erlaubt nur eine gleichzeitig ausgeführte Aktivität pro Ressource.

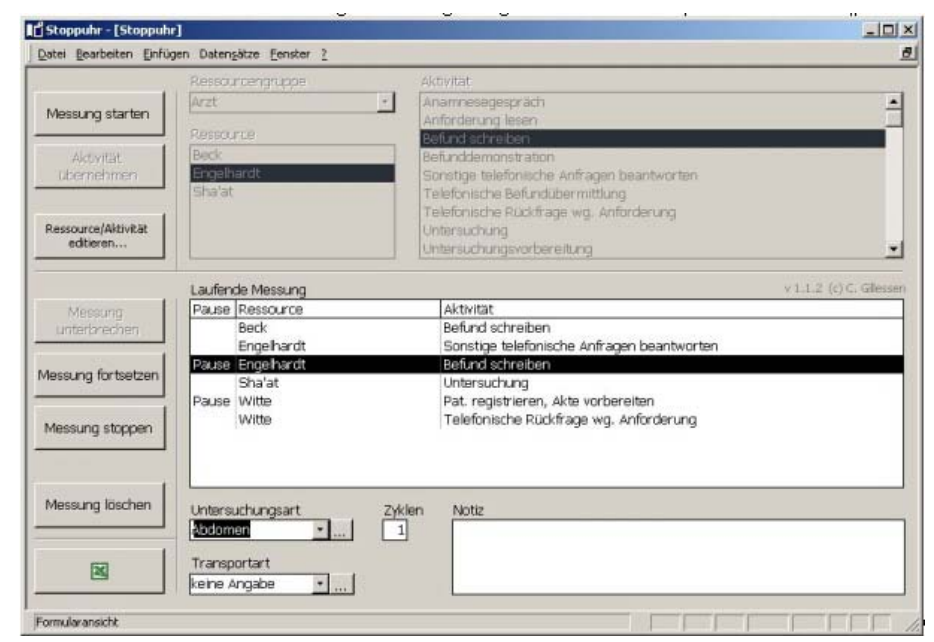

Abbildung 4-11: Bedienungsoberfläche der proprietären Software zur Zeitmessung Unabhängig von den Zeitmessungen wurde eine Strichliste mit jenen Vorgängen geführt, die nicht fest an die Projektstruktur gebunden waren (Typ lb und Typ Ilb). Für jeden Vorgang wurde gezählt, wie häufig er pro Zeiteinheit auftrat. Dieser Wert wurde 
über die durchschnittliche Projektdauer (Untersuchungsdauer) in die durchschnittliche Anzahl Vorgänge pro Untersuchung umgerechnet.

\section{Aufbereitung der Messwerte:}

Für jede Vorgangsdauer wurden die optimistischen, die realistischen und die pessimistischen Zeiten bestimmt. Als optimistische Zeit wird dabei die jeweils kürzeste gemessene Zeit eingesetzt, als pessimistische Zeit die jeweils längste.

\subsubsection{Phase III: Netzplan anfertigen}

In der dritten Phase erfolgte die Abbildung des Projektes in einem CPM-Netzplan. Die zeitlichen Zusammenhänge wurden graphisch im Gantt-Diagramm dargestellt. Je nach Abhängigkeiten der Einzelaktivitäten werden mehrere parallele Teilprojekte erstellt, die nicht logisch zusammenhängen. Es wurden mehrere Netzplanvarianten erstellt. Dafür wurden die Begriffe der optimistischen, realistischen und pessimistischen Betrachtung eines Netzplans von der Vorgangsdauer auf die Projektstruktur ausgedehnt. In der optimistischen Darstellung enthielt der Netzplan somit nur die Vorgänge, die regelmäßig in jeder Untersuchung auftreten (Typ la/lla), mit den jeweils kürzesten gemessenen Zeiten. In die realistische Darstellung fanden alle regelmäßig in jeder Untersuchung auftretenden Vorgänge und jene Vorgänge, deren Auftretenshäufigkeit über $80 \%$ lag, Eingang. Die Vorgangsdauern entsprachen den berechneten realistischen Zeiten. In der pessimistischen Darstellung wurden alle beobachteten Vorgänge mit ihren jeweils längsten gemessenen Zeiten berücksichtigt. Der Netzplan wurde mit einer Projektplanungssoftware erstellt.

\subsubsection{Phase IV: Auswertung}

Phase IV beginnt mit der Berechnung des jeweils frühesten und spätesten Zeitpunkts für den Beginn eines Vorgangs (ES (Early Start) und LS (Late Start)). Anschließend werden der früheste und späteste Zeitpunkt für die Beendigung eines Vorgangs ermittelt (EF (Early Finish) und LF (Late Finish)). Diese Berechnungen werden für jeden Vorgang durchgeführt. Auf der Grundlage dieser Daten wurden die Gesamtprojektdauer und die Zeitreserven unter optimistischer, realistischer und pessimistischer Betrachtung 
ermittelt. Die Zeitreserven umfassen Puffer- und Schlupfzeiten:

1. Die Pufferzeit gibt den Zeitraum an, um welchen ein Vorgang verlängert werden kann, ohne die Gesamtuntersuchungsdauer zu überschreiten. Die Pufferzeiten der einzelnen Arbeitsschritte werden dadurch berechnet, dass von inrem LF-Wert der dazugehörende EF-Wert subtrahiert wird (FP = LF $E F)$.

2. Schlupfzeiten beschreiben den Zeitraum, um den ein Ereignis auf später verschoben werden kann, ohne die Gesamtdauer der Untersuchung zu verlängern $(S Z=L S-E S)$.

Anschließend wurde eine Engpassanalyse durchgeführt mit Berechnung der kritischen Vorgänge und Ereignisse. Vorgänge sind kritisch, wenn ihre Pufferzeit 0 ist, d.h. diese Arbeitsschritte besitzen keine Zeitreserven. Ereignisse (z.B. der Beginn eines Vorgangs) sind kritisch bei einer Schlupfzeit von 0, d.h. sie können nicht verschoben werden.Alle Berechnungen wurden mit Projektplanungssoftware automatisiert. 


\subsubsection{Ergebnisse: Netzplan für den Ultraschall}

Routinearbeitsplatz

Die im Funktionsbereich durchgeführte Ultraschalluntersuchung umfasste insgesamt 10 Vorgänge. Der Arzt führt davon 4 Vorgänge durch und die Schwester 6. Die einzelnen Vorgänge mit ihren Vorgänger-Nachfolger-Beziehungen und ihren durchschnittlichen Dauern sind in Tabelle 4-4 aufgezeigt. Alle Arbeitsschritte bis auf eine Ausnahmen können beginnen, wenn der unmittelbare vorherige Arbeitsschritt abgeschlossen ist. Der Vorgang h (Dokumentation bzw. schriftlicher Befundtext) kann erst beginnen, wenn die Arbeitsschritte e (Ultraschalluntersuchung) und b (Akte anlegen) abgeschlossen sind.

Tabelle 4-3: Auflistung der Arbeitsvorgänge mit Vorgänger und Ressourcenallokation (Funktionsbereich 1; (to: optimistische Zeit , $t_{r}$ : realistische Zeit, $t_{p}$ : pessimistische Zeit))

\begin{tabular}{|c|c|c|c|c|c|c|}
\hline Vorgang & Beschreibung & Ressource & Vorgänger & $\begin{array}{l}t_{0} \\
{[\min : s]}\end{array}$ & $\begin{array}{l}\mathrm{t}_{\mathrm{r}} \\
{[\mathrm{min}: \mathrm{s}]}\end{array}$ & $\begin{array}{l}t_{p} \\
{[\min : s]}\end{array}$ \\
\hline A & Patient abrufen & Schwester & & $0: 20$ & $0: 58$ & 2:01 \\
\hline$B$ & $\begin{array}{l}\text { Patient registrieren } \\
\text { Akte vorbereiten }\end{array}$ & Schwester & $A$ & $0: 08$ & $2: 56$ & $9: 10$ \\
\hline C & $\begin{array}{l}\text { Untersuchungsvorbereitung: } \\
\text { Raum reinigen } \\
\text { Patient ins Zimmer } \\
\text { begleiten } \\
\text { Daten am Sonogerät } \\
\text { eingeben }\end{array}$ & Schwester & A & $1: 21$ & $3: 59$ & 9:10 \\
\hline D & Kurzanamnese & Arzt & C & $0: 15$ & $0: 24$ & $0: 32$ \\
\hline$E$ & Ultraschalluntersuchung & Arzt & $D$ & $3: 13$ & $12: 49$ & $30: 41$ \\
\hline$F$ & Verabschiedung & Arzt & $E$ & $0: 19$ & $1: 34$ & $4: 38$ \\
\hline G & $\begin{array}{l}\text { Patient aus dem Zimmer } \\
\text { begleiten }\end{array}$ & Schwester & $F$ & $1: 30$ & $2: 47$ & 4:03 \\
\hline $\mathrm{H}$ & Dokumentation & Arzt & $b, f$ & $1: 44$ & $8: 22$ & $18: 42$ \\
\hline I & $\begin{array}{l}\text { Nachbereitung: } \\
\text { Untersuchung abrechnen } \\
\text { Befund in Akte sortieren }\end{array}$ & Schwester & $b, h$ & $0: 35$ & $3: 54$ & 10:57 \\
\hline $\mathrm{J}$ & $\begin{array}{l}\text { Befund aushändigen } \\
\text { Patient versenden }\end{array}$ & Schwester & 1 & 0:09 & $2: 13$ & 5:01 \\
\hline
\end{tabular}


Die Ergebnisse der Zeitmessung bei 30 Ultraschalluntersuchungen sind in Tabelle 43 als die jeweils kürzest möglichen Zeiten für die Durchführung der einzelnen Arbeitsschritte angegeben (optimistische Vorgangsdauer). In Abbildung 4-12 und 4-13 sind die auf den Ergebnissen der Phase 1 und 2 beruhenden Gantt-Diagramme bei optimalen und realistischen Bedingungen dargestellt. Aus innen ergibt sich eine kürzest mögliche Zeit (optimistische Betrachtung) von 7:56 Minuten und eine zu erwartende Untersuchungszeit (realistische Betrachtung) von 34:13 Minuten für die Durchführung einer kompletten Ultraschalluntersuchung (Gesamtprojektdauer) einschließlich Vor- und Nachbereitung des Patienten und Erstellung eines schriftlichen Befundes. Für die eigentliche Ultraschalluntersuchung durch den qualifizierten Facharzt werden dabei 3:13/12:49 Minuten (optimistisch/realistisch) benötigt.

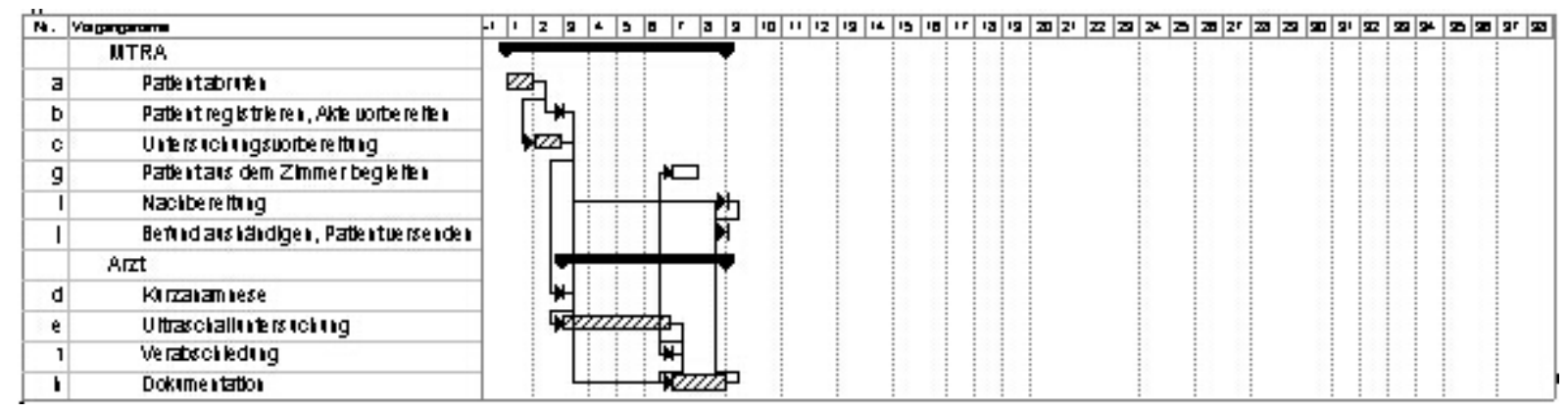

Abbildung 4-12: Funktionsbereich 1, optimistische Betrachtung

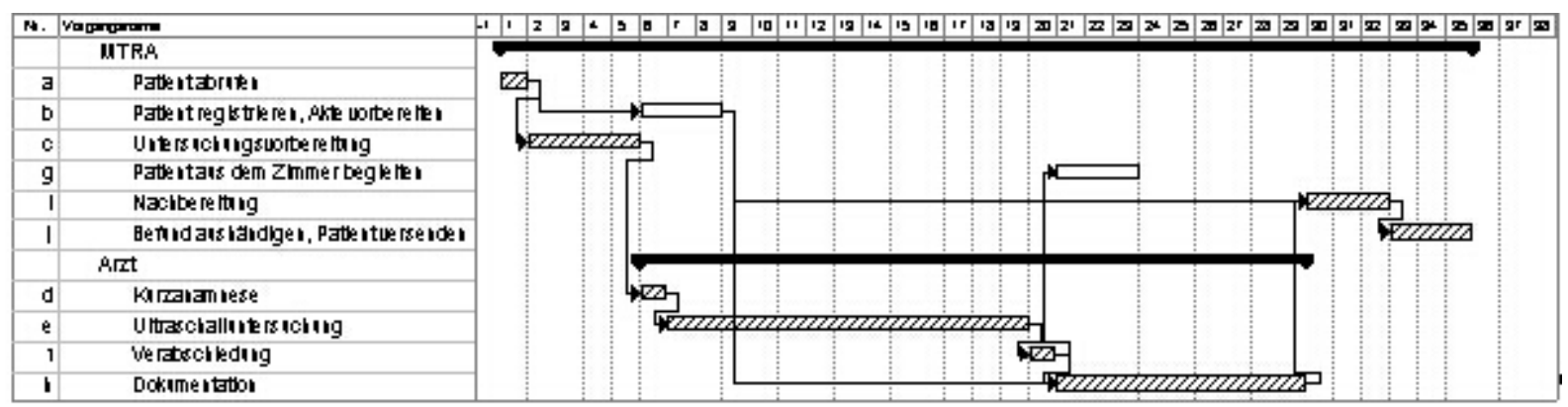

Abbildung 4-13: Funktionsbereich 1, realistische Betrachtung

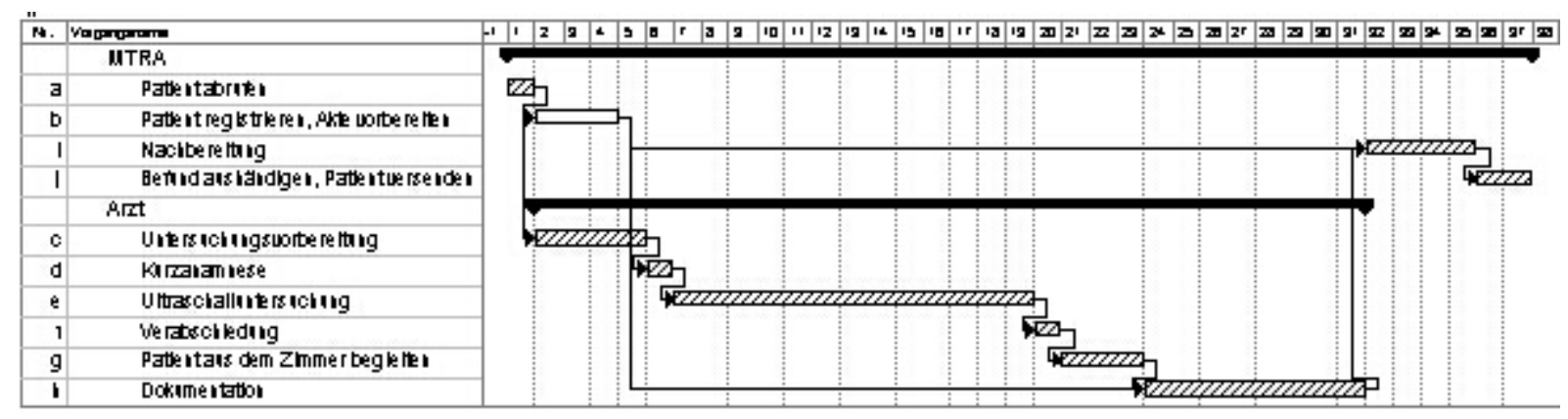

Abbildung 4-14: Funktionsbereich 2, realistische Betrachtung 
Um die optimistische Gesamtprojektdauer von 7:56 Minuten einhalten zu können, müssen die Arbeitsschritte $a, c, d, e, f, i, j$, und $h$ in der kürzest möglichen Zeit durchgeführt werden. Dies entspricht dem kritischen Weg. Die Arbeitsschritte des kritischen Weges besitzen keine Pufferzeit, auch wenn sie zum frühest möglichen Anfangszeitpunkt beginnen. Die Arbeitsschritte des Arztes befinden sich alle auf dem kritischen Pfad. Somit bestehen für den Arzt keine Zeitreserven bei der Untersuchung. Anderes verhält es sich bei der Krankenschwester: Nur die Arbeitsschritte a, c, i und j befinden sich auf dem kritischen Pfad. Zwei Vorgänge sind nicht kritisch und können parallel zur Tätigkeit des Arztes durchgeführt werden. Es bestehen somit für die Krankenschwester vor Beginn des Vorgangs b (Patient registrieren, Akte vorbereiten) eine freie Pufferzeit von 3:53 Minuten bei optimistischer Betrachtung bzw. 23:09 Minuten bei realistischer Betrachtung. Zum Vergleich zeigen wir einen Untersuchungsablauf, den wir im internistischen Funktionsbereich für Ultraschall in einer anderen Klinik beobachtet haben. Die einzelnen Vorgänge stimmten dabei mit den eingangs beschriebenen überein; d.h. es werden in beiden Funktionsbereichen die gleichen Arbeitsschritte für eine Ultraschalluntersuchung durchgeführt. Die Ressourcenzuteilung ist indes unterschiedlich. Die Vorgänge c (Untersuchungsvorbereitung) und g (Patient aus dem Untersuchungszimmer begleiten) sind hier dem Arzt zugeordnet. In den Abbildungen 3.-12 und 3.-13 sind die beiden Arbeitsabläufe bei realistischer Betrachtung zum Vergleich dargestellt. Die Gesamtuntersuchungsdauer erhöht sich auf 37:00 Minuten, die Anzahl der kritischen Vorgänge erhöht sich für den Arzt, und die Pufferzeit für die Schwester verlängert sich von 23:09 auf 25:56 Minuten.

\subsubsection{Diskussion}

Um die Krankenhausaufenthaltsdauer für Patienten möglichst gering zu halten, ist es unumgänglich, einen strengen Terminkalender für die notwendigen Untersuchungen und therapeutischen Maßnahmen einzuhalten. Dazu müssen diese in den Funktionsbereichen kurzfristig und zügig durchgeführt werden, was effiziente Abläufe voraussetzt. Die NPT ist eine Möglichkeit zur Analyse der eigenen Arbeitsabläufe. Bei ihrer Anwendung kristallisiert sich häufig heraus, dass ein Arbeitsablauf unter verschiedenen Gesichtspunkten optimiert werden kann. Entscheidend ist, welche 
Prioritäten gesetzt werden; so kann man den Ablauf einerseits patientenorientiert auf eine möglichst kurze Aufenthaltsdauer für den Patienten hin optimieren oder anderseits kostenorientiert auf eine möglichst effiziente Auslastung der Ressourcen. In den seltensten Fällen können in einer Projektkonfiguration alle Prioritäten gleichzeitig erfüllt

werden.

Die verschiedenen zeitlichen Betrachtungen ermöglichen es, Reserven und mögliche Schwächen des Arbeitsablaufs zu verdeutlichen. Optimistische und pessimistische Betrachtungen sind zwar theoretische Werte, da niemals in einem Ablauf alle Vorgänge mit den kürzest bzw. längst gemessenen Vorgangsdauern durchlaufen werden. Jedoch zeigt die optimistische Betrachtung, wie kurz ein Arbeitsablauf bei optimalen Bedingungen sein kann und bietet Anhaltspunkte und Ansporn zu Verbesserungen. Umgekehrt zeigt die pessimistische Betrachtung Quellen für Verzögerungen auf. Für die Darstellung und Analyse des derzeitigen Zustandes wie z.B. des kritischen Pfades orientiert man sich an den realistischen Werten. In unserem Beispiel liegen alle Vorgänge des Arztes auf dem kritischen Pfad. Das ist darauf zurückzuführen, dass die ärztlichen Tätigkeiten von den administrativen Tätigkeiten der Schwester eingerahmt sind. Einerseits kann der Arzt nicht mit seiner Untersuchung beginnen, bis bestimmte administrative Voraussetzungen erfüllt sind. Andererseits muss die Schwester mit der Vervollständigung der Untersuchung warten, bis der Arzt seine zeitintensiven Vorgänge beendet hat. Daraus ergeben sich die Pufferzeiten für die Schwester. Anhand von Veränderungen der Vorgänge des kritischen Pfades kann die Gesamtuntersuchungszeit verändert werden. Dies wird im Vergleich der beiden Funktionsbereiche deutlich. Im eingangs beschriebenen Funktionsbereich 1 werden die meisten untersuchungsbegleitenden Maßnahmen von den Schwestern durchgeführt, während sich der Arzt auf die Untersuchung konzentriert. Im zweiten Funktionsbereich übernimmt der Arzt die Untersuchungsvorbereitung und die Begleitung des Patienten aus dem Untersuchungsraum, während sich die Schwester auf administrative Tätigkeiten beschränkt. In unserem Beispiel ist die Verzögerung der Gesamtuntersuchung gering, da die untersuchungsvorbereitenden Maßnahmen ohnehin durchgeführt werden müssen bevor der Arzt mit der Untersuchung beginnen kann.

Der tatsächliche Einfluss von Veränderungen auf die Effizienz eines Arbeitsablaufs zeigt sich in der Regel erst, wenn mehrere Arbeitsabläufe hintereinander 
durchgeführt werden. Die Ursache dafür liegt in der Überlappung der einzelnen Untersuchungen. Bestimmte Vorgänge der Nachfolgeuntersuchung können bereits durchgeführt werden, während die aktuelle Untersuchung noch läuft. So kann die Schwester z.B. bereits die Akte des nächsten Patienten vorbereiten, während der Arzt noch den Befund des letzten Patienten schreibt. Dies ist möglich, weil die Schwester in unserem Beispiel innerhalb einer Untersuchung Pufferzeiten hat, die sie mit den Vorgängen der Nachfolgeuntersuchung auffüllen kann. Hier deutet sich bereits an, dass die Ressourcenverteilung im Funktionsbereich 2 Nachteile hat, da die Tätigkeiten des Arztes vollständig den kritischen Weg bestimmen. In diesem Zusammenhang hat die NPT Einschränkungen. Sie wurde entwickelt, um singuläre Abläufe abzubilden, wie sie im Rahmen von Projekten vorkommen. Eine Analyse sich wiederholender Arbeitsabläufe mit einem hohen Anteil an Überlappung wie der Ultraschalluntersuchung ist möglich, aber aufwändig. Hier werden mehrere aufeinanderfolgende Untersuchungen in einem Netzplan abgebildet, um Interdependenzen transparent zu machen. Veränderungen in der Abfolge der Vorgänge, deren Einfluss auf den Gesamtverlauf untersucht werden soll, werden in jedem Einzelablauf vorgenommen, entsprechend steigt der Aufwand mit der Anzahl der betrachteten Untersuchungen. Eingeschränkt beurteilbar ist auch der Einfluss von Vorgängen, die nur gelegentlich und an unterschiedlichen Stellen auftreten (z.B. Telefonate). Sie können in die pessimistische Betrachtung eingebaut werden, um einen Eindruck von ihrer Bedeutung für den Arbeitsablauf im Vergleich zur optimistischen oder realistischen Betrachtung zu gewinnen. Ihr statistisch korrekter Einfluss lässt sich damit jedoch nicht ermitteln. 


\subsubsection{Schlussfolgerungen}

Die Netzplantechnik stellt ein Werkzeug im Rahmen der Prozessoptimierung dar, mit dem Arbeitsabläufe hinsichtlich Effizienz bei gegebener Ergebnisqualität analysiert und optimiert werden können. Ihre Anwendung erhöht die Transparenz in Arbeitsabläufen; durch Zerlegung derselben in Einzelschritte und Betrachtung ihrer Abhängigkeiten werden Schwachpunkte und Verzögerungen deutlich. Der Einfluss von Veränderungen im Ablauf oder in der Ressourcenzuteilung kann vorab geschätzt werden, ebenso kann der Ressourcenbedarf für einen Arbeitsdurchgang abgeschätzt werden. Einschränkungen in der Handhabbarkeit zeigt die Netzplantechnik bei Betrachtung repetitiver Abläufe mit hohem Anteil an Überlappung und in der Aussagekraft bei der Abbildung nicht regelmäßig auftretender Vorgänge. 


\subsection{Anwendung der Netzplantechnik in der Computertomographie}

\subsubsection{Einleitung}

Um jedem Patienten Zugang zu der individuell besten Diagnostik und Therapie zu gewährleisten, ist ein wirtschaftlicheres Denken im Gesundheitssystem unumgänglich. Der Einsatz von privatwirtschaftlicher Steuerungsinstrumente wird mit dem Ziel einer verbesserten Patienten- und Serviceorientierung und der Beseitigung von Management- und Steuerungsdefiziten intensiv diskutiert. Insbesondere durch Einführung eines DRG-Systems zur pauschalisierten, fallbezogenen Kostenvergütung im Krankenhaussektor repräsentiert eine wesentliche Maßnahme zur Kostendämpfung. Verlängerte Liegedauern und Wartezeiten sind, sofern deren Ursache in mangelnden wirtschaftlich-organisatorischen Maßnahmen zu suchen ist, im Sinne des Patienten, des Kostenträgers und auch des Krankenhauses zu verkürzen. Prozessmanagement ist ein relativ junges Instrument zur Planung, Steuerung und Kontrolle der Leistungserstellung. Eine verbesserte Transparenz wird im Gesundheitswesen durch eine EDV-basierte Informationstechnologie erreicht. Im weiteren gewinnt der Patient als Kunde an Bedeutung, Wettbewerb und Patientenorientierung werden im klinischen Alltag immer relevanter. Aspekte wie diese sind auch für die Radiologie von Bedeutung, die mit der Computertomographie (CT) einen für alle Bereiche der Medizin unverzichtbaren Standard der Diagnostik stellt. Die CT stellt einen Arbeitsbereich mit einer großen Anzahl an täglichen Untersuchungen und großen Pattientendruchsatz dar. Um eine effiziente Geräteauslastung $z u$ erreichen und den steigenden klinischen Anforderungen an Untersuchungen $z u$ entsprechen, ist ein täglicher Mehrschichtbetrieb vielerorts inzwischen Normalität.

Die Computertomographie verursacht zwar relativ hohe Kosten pro Untersuchung, durch ihre Aussagekraft resultiert allerdings ein Nutzen, der den Aufwand anderer, eventuell zahlreicherer Untersuchungen vermeidet. Insgesamt steht somit eine Kostenersparnis im Vordergrund. Diese Arbeit untersucht die Eignung von Netzplantechnik für den Funktionsbereich Computertomographie einer radiologischen Klinik.

\subsubsection{Patientenorientierte Optimierung der Arbeitsprozesse}

Um die Patientenzufriedenheit zu steigern, bietet sich in der Radiologie nicht nur der 
förmlich-höfliche Umgang mit den Patienten, sondern auch eine im Sinne des Patienten und auch des Klinikbudgets gezielte Ablauforganisation an. Der aus der Industrie stammende Begriff Produktionsprozess richtet sich in der vorliegenden Studie auf Untersuchungen im Funktionsbereich der Computertomographie. Dabei wird der Patient die entscheidende Eingabe (Input) und Ausgabe (Output). Ziel ist aus seiner Sicht, die Wartezeit zu reduzieren.

Das Leitmotiv des Prozessmanagements ist die Kunden-/ bzw. Patientenorientierung. Sie gilt als Voraussetzung zur Erlangung von Wettbewerbsvorteilen. In diesem Verständnis soll der Patient als Kunde gesehen werden. Das Maß für die Kundenorientierung ist die Kundenzufriedenheit. Nach Gaitanides bedeutet dies: „...keine Abweichung zuzulassen von den identifizierten Kundenwünschen“ (34). Wenn Prozessmanagement auf ein neues Anwendungsgebiet übertragen wird, ist es notwendig, den Grundgedanken der Kundenorientierung entsprechend zu interpretieren. Zahlreiche Unternehmen wissen um die Kundenorientierung als Schlüsselfaktor zur Sicherung eines langfristigen Erfolgs. Sie nimmt in den Zielsystemen vieler Unternehmen der verschiedensten Branchen heute einen hohen Stellenwert ein (88). Da die Konkurrenz der Krankenhäuser untereinander immer mehr zunimmt, wird es immer wichtiger, den Patienten als Kunden zu begreifen, an dessen Zufriedenheit es sich zu orientieren gilt, was momentan keineswegs eine Selbstverständlichkeit ist. Kunden- bzw. patientenorientiertes Denken und Handeln erfordert, die Bedürfnisse der Zielgruppe zu identifizieren. Nach Homburg geschieht dies in Bezug auf die erhaltene Leistung (das „Was“ besser: was wird erreicht?) und in Bezug auf die Interaktion (das „Wie“ besser: wie wird es erreicht?) (88). Nur wenn der Kunde mit dem „Was“ und „Wie“ der Transaktion zufrieden ist, wird die Gesamtzufriedenheit ein hohes Niveau erreichen. Daher gilt es, beide Dimensionen zu maximieren, wenn die Kundenorientierung ein maßgebliches Ziel sein soll. Ein hoher Zufriedenheitsgrad seiner Kunden versetzt den Anbieter in die Lage, seine primären Ziele zu erreichen, nämlich Umsätze und Marktanteile zu steigern (85). So wird die Abhängigkeit von Kunde (Patient) und Anbieter (Krankenhaus) voneinander zu beiderseitigem Vorteil gekehrt. Im Mittelpunkt der Prozessorientierung stehen Prozesse, worunter die Aktivitäten der Leistungserstellung zu verstehen sind, die fortschrittsbezogen zusammengefasst wurden. Die Strukturierung des Unternehmensgeschehens in Prozesse ermöglicht eine Verfolgung und Optimierung aller Aktivitäten, die zur Erstellung eines Produktes oder einer Dienstleistung 
erforderlich

$\operatorname{sind}(85)$.

Die Ganzheitlichkeit ist ein Charakteristikum des Prozessmanagements und auch des Total Quality Management (TQM). Das Total Quality Management zielt auf eine umfassende Qualitätskontrolle aller Unternehmensbereiche mit dem Ziel, dadurch eine kontinuierliche Effizienzsteigerung und Kostenreduktion zu erreichen (19), wobei die Kundenzufriedenheit in den Mittelpunkt gestellt ist (32). TQM wird zum Teil unterschiedlich definiert: "Total Quality Management ist ein langfristig angelegtes, integriertes Konzept und ein System von Prinzipien und praktischen Instrumenten, mit deren Hilfe die Effizienz der internen Prozesse und die Qualität der Produkte und Dienstleistungen kontinuierlich verbessert werden sollen, um eine optimale Bedürfnisbefriedigung der Kunden zu ermöglichen“ (68). „Total Quality Management ist eine auf der Mitwirkung ihrer Mitglieder beruhende Führungsmethode einer Organisation, die Qualität in den Mittelpunkt stellt und durch Zufriedenheit ihrer Kunden auf langfristigen Geschäftserfolg sowie auf Nutzen für die Mitglieder der Organisation und für die Gesellschaft zielt“. Im Sinne des Prozessmanagements versteht man darunter die Steuerung aller Unternehmensprozesse unter Kosten-, Qualitäts-, und Zeitaspekten mit dem Ziel, alle vom Kunden wahrgenommenen Leistungsmerkmale zu berücksichtigen (33). Das Zusammenspiel von Instrumenten und Methoden des Kosten-, Qualitäts-, TimeBased-Managements (s.u.) und der Organisationslehre ermöglicht die Realisierung der Ganzheitlichkeit. Zur praktischen Umsetzung dessen bedarf es der Entwicklung neuer bzw. Modifikation bestehender, prozessorientierter Controllinginstrumente. Zur langfristigen Orientierung des Verbesserungsprozesses: durch herkömmliche Rationalisierungsprojekte, z.B. unter Anwendung des Business Reengineerings, lassen sich meist nur kurzfristige Erfolge der Verbesserung der Wettbewerbsfähigkeit erreichen. Das Prozessmanagement baut auf einen kontinuierlichen Verbesserungsprozess in kleinen Schritten, auf ein sogenanntes Continuous Improvement. Es soll die Wettbewerbsfähigkeit kontinuierlich an die sich ändernden Bedingungen anpassen (85). Die Verantwortung für das Erreichen von Verbesserungen wird Prozessverantwortlichen übertragen. Die Motivation zur Durchführung von Prozessverbesserungen wird durch die Festlegung von SollGrößen unterstützt (33). 


\subsubsection{Netzplantechnik}

Netzpläne sind ein wichtiger Bestandteil des Projektmanagements, sie tragen entscheidend zum Planen, Analysieren und Steuern von Projektabläufen bei. Von verschiedenen Netzplantechnikmethoden haben sich für den Bereich der Medizin die deterministischen durchgesetzt, d.h. alle im Netzplan dargestellten Wege müssen durchlaufen werden und die Abläufe sind vorher bestimmbar. In diese Kategorie fallen:

1. CPM (Critical Pathway Method), 1957, USA

2. PERT (Program Evaluation and Review Method), 1957, USA. Wobei anzumerken ist, dass PERT mit der Drei-Zeiten-Schätzung (s.u.) ein stochastisches Element enthält.

3. MPM (Metra Potential Method),1959, Frankreich

Ein Netzplan setzt sich zusammen aus

1. Vorgängen ( = zeiterforderndes Geschehen mit definiertem Anfang und Ende)

2. Ereignissen ( = definierte Zustände)

3. Anordnungsbeziehungen ( = Reihenfolgebedingungen / Abhängigkeiten der Vorgänge untereinander innerhalb des Projektablaufes )

\subsubsection{Erfahrungen mit CPM und PERT in der klinischen Medizin}

CPM und PERT sind eng verwandte Planungs- und Management-Methoden und können daher als CPM/PERT zusammen genannt werden (62). In mehreren Studien wurde die Reduktion von Liegedauern nach Anwendung der Critical Pathway Method dokumentiert $(15 ; 27 ; 104)$. Die Autoren berichten von verbesserter Kommunikation zwischen Ärzten untereinander und dem Pflegepersonal, was allerdings eine schwer messbare Tatsache ist (14). Gutes Teamwork und funktionierende Kommunikation sind äußerst wichtig für das Gelingen. Die Einsatzgebiete und Funktionen der einzelnen Klinikangestellten müssen im Rahmen des Protokolls klar festgelegt werden, um dessen möglichst reibungslosen Ablauf zu gewährleisten (104). Bei Patienten stößt CPM auf Akzeptanz. Wie bei Mosher et al. berichten, kann es auf den Patienten angstreduzierend wirken, zu wissen was genau inn an welchem Tag erwartet. Auch kann so die Motivation steigen, an allen vorgesehenen Untersuchungen und Maßnahmen teilzunehmen. Auch die Angehörigen werden über den Behandlungsplan informiert und damit involviert, was die Patientenzufriedenheit 
zu steigern vermag $(64 ; 70)$. Luttman et al. verweisen darauf, dass Vorteile, die mit CPM/PERT geschaffen werden zum Teil nur mit relativ großem Aufwand erreicht werden können. Denn in die Behandlungsprozesse eines Patienten können diverse Interaktionen zwischen verschiedensten Leuten aus verschiedenen Kliniken einfließen. So können multidisziplinäre Teams Wochen zur Entwicklung eines Pathways benötigen und Varianzen können störend sein. Weiterhin wird festgestellt, dass $80 \%$ des Vorkommens von Problemen nur durch 20\% der möglichen Ursachen ausgelöst werden. Es ist also sinnvoll, die nicht allzu vielen Störfaktoren zu identifizieren, die beispielsweise den Klinikaufenthalt eines Patienten verlängern (56). Varianzen sind Abweichungen vom kritischen Weg, sie können positiv oder negativ, vermeidbar oder unvermeidbar sein. Man muss sie berücksichtigen und ggf. in den Prozessfluss einbauen (14). Diese unvorhergesehenen Abweichungen vom kritischen Weg müssen als Schlüsselereignisse erkannt, dokumentiert, analysiert und dann weitestgehend reduziert werden. Die Multikolinearität ist durch die Vernetzungen und Abhängigkeiten der unterschiedlichen Behandlungsstufen bedingt (70). Wenn eine Aktivität verspätet beendet wird, hat dies Auswirkungen auf alle folgenden Aktivitäten, die Verzögerung zieht sich kaskadenartig durch den gesamten Prozess. Diese Beziehungen sind mit PERT/CPM darstellbar, dadurch können Lösungsmöglichkeiten entwickelt werden

Luttman et al. schlagen den PDCA-cycle zur kontinuierlichen Qualitätsverbesserung vor: 


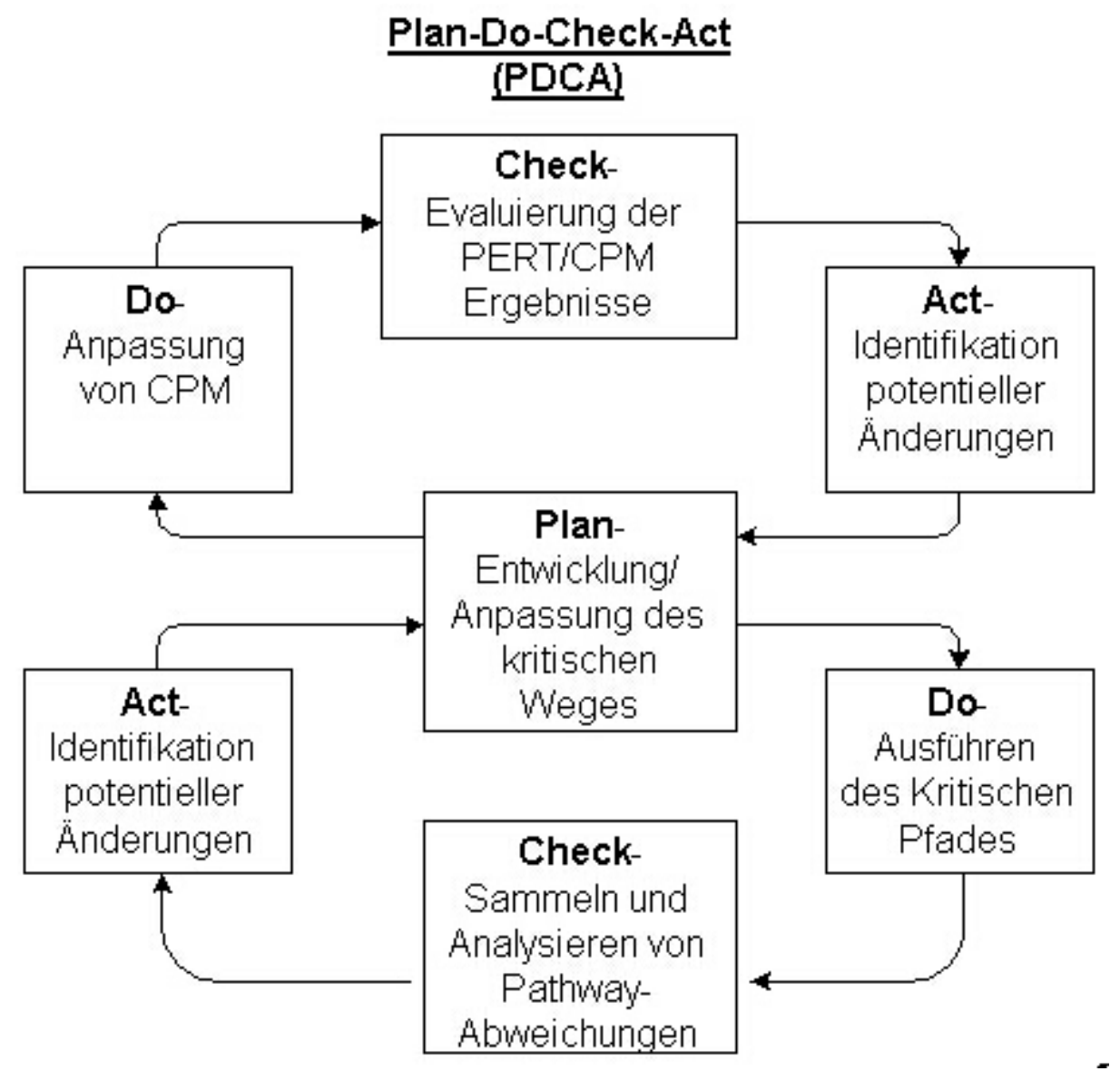

Abbildung 4-15: Planungs- und Durchführungs- und Überprüfungszyklus für einen PERT/CPM Netzplan

Damit wird Qualitätsmanagement direkt ans Krankenbett gebracht, es ist als planendes, kommunizierendes und koordinierendes Instrument zu sehen, welches sich ändernden Gegebenheiten anzupassen vermag. Es gibt allerdings auch begründete Bedenken über CPM/PERT. Multimorbide Patienten oder das Auftreten von Problemen lassen sich oft nicht auf einen einzigen standardisierten Pfad reduzieren $(30 ; 79 ; 103)$. Wobei die Berichte in ihrer Einschätzung teils sehr voneinander abweichen. Beispielsweise konnte in der von Falconer et al. publizierten Studie mit CPM kein Effekt auf Kosten, Länge des Krankenhausaufenthaltes und Behandlungserfolg erzielt werden (29). Andere hingegen äußern sich positiv in Bezug auf die genannten Punkte (49; 54). Die Methoden des kritischen Weges bewegen sich durch Standardisierung hin zu mehr Qualität und Effektivität, was den Arzt sich in seiner Autonomie eingeschränkt sehen lassen kann. Zudem kann die Besorgnis entstehen, dass es dem Arzt angelastet wird, wenn er den Patienten nicht dem jeweiligen Pfad entsprechend behandelt oder behandeln kann. Da tendenziell jede Änderung von 
zahlreichen Bedenken begleitet wird, sind offene Diskussionen notwendig. Zudem sollte jeder Arzt die Möglichkeit haben, Vorschläge zur Änderung des Pfades generell oder speziell für einzelne Patienten zu machen (70).

\subsubsection{Fragestellung}

Ziel der prozessorientierten Gestaltung der Krankenhausorganisation ist es, den Nutzen aller Einzelleistungen und damit auch der Gesamtleistung des Krankenhauses für den Patienten zu verbessern (25). In der vorliegenden Studie soll daher der Teilprozess „Untersuchung des Patienten im Computertomographen“ prozessorientiert interpretiert werden und als Beispiel für weitere Organisationen im Krankenhaus dienen. Für den Funktionsbereich der Computertomographie haben sich seit der klinischen Einführung in den letzten 20 Jahren wesentliche technische und organisatorische Veränderungen vollzogen. Die Geräte sind schneller geworden, das Personal wurde im Umgang und dem Betrieb geschult und Untersuchungsprotokolle wurden kontinuierlich verbessert. Gleichzeitig ist die Anzahl der Untersuchungen und der klinischen Fragestellungen stetig gewachsen. Die Organisation der Untersuchung bestimmt zusehends die optimale Auslastung der kostenintensiven Geräte in Krankenhaus und

Praxis.

Es wird eine Zeitanalyse von CT-Untersuchungen an einem Universitätskrankenhaus unter Verwendung der CPM (Critical Pathway Method) $(12 ; 14 ; 41 ; 53 ; 96)$ und PERT (Program Evaluation and Review Technique) vorgestellt (56). Unser Ziel war es, eine Übersicht über die komplexen Arbeitsabläufe zu erhalten, diese untereinander mit verschiedenen Computertomographen, verschiedenen Patientenkategorien und verschiedenen Arbeitsteams zu vergleichen, um Arbeitsabläufe schneller, verständlicher, effizienter und damit kosteneinsparender durchführen zu können. Die Analyse von Arbeitsabläufen beschränkt sich Einzeilenspiral- CT-Geräten, da die Mehrzeilen-CT-Geräte erst nach Abschluss der Datenerhebung in die klinische Routine eingeführt wurden.

\subsubsection{Methodik}

\subsubsection{Beschreibung der CT-Arbeitsplätze}

Es wurden zwei Arbeitsbereiche mit unterschiedlichen Geräten in der radiologischen Diagnostik der Klinik für Strahlenheilkunde am Universitätsklinikum Charité Campus 
Virchow-Klinikum in Berlin untersucht. Beide Geräte befanden sich bezüglich der Raumaufteilung in vergleichbaren Arbeitsumgebungen. Die räumliche Verteilung umfasste einen Wartebereich für Patienten, einen Untersuchungsraum und einen Kontrollraum, in dem das CT bedient wurde. Die Weiterverarbeitung der Daten, der Ausdruck und das anschließende Befunden der Bilder fanden ebenfalls im Kontrollraum statt.

\subsubsection{Patientenkategorien}

1. Mobile Patienten (stationär und ambulant)

2. Immobile, d.h. bettlägerige Patienten (aufklärungsfähig)

3. Intensivmedizinische Patienten

\subsubsection{Untersuchungskategorien}

CT des Kopfes ohne und mit Kontrastmittel

1. Schichtkollimation Schädel-Basis: $5 \mathrm{~mm}$

2. Schichtkollimation Neurocranium: $8 \mathrm{~mm}$

3. Röhrenstrom: $400 \mathrm{mAs}$

4. Röhrenspannung: $140 \mathrm{kV}$

CT des Thorax nur mit Kontrastmittel

1. Schichtkollimation: $5 \mathrm{~mm}$

2. Tischvorschub: $7,5 \mathrm{~mm}$

3. Röhrenstrom: $180 \mathrm{mAs}$

4. Röhrenspannung: $140 \mathrm{kV}$

Die Untersuchungsprotokolle umfassten folgenden Ablauf:

1. Erstellen eines Tomogramms

2. Einstellen der Schichtebenen

3. (nur bei CT Kopf): Erster Untersuchungsdurchgang ohne Kontrastmittel

4. Gabe von Kontrastmittel

5. Untersuchungsdurchgang mit Kontrastmittel

\subsubsection{Erstellen des Prozessmodells}

Phase I-Strukturermittlung

Das Projekt wurde nach Rücksprache mit leitenden Mitarbeitern, die unmittelbar am CT-Arbeitsprozess beteiligt waren, beschlossen (Oberärzte, Leitende MTRA, Assistenzärzte). Projektbesprechungen fanden im Rahmen mehrerer Treffen mit den Beteiligten statt. 
In Phase I wurde der Untersuchungsablauf in anfallende Arbeitsschritte (=Vorgänge) einer Untersuchung am CT-Arbeitsplatz eingeteilt. Ein neutraler Beobachter dokumentierte dafür während 2 Wochen den Ablauf der Untersuchung und definierte die anfallenden Arbeitsschritte. Die Vorgänge wurden den einzelnen Arbeitskräften zugeordnet und nach ihrem zeitlichen Ablauf nummeriert. Die einzelnen Vorgänge wurden nach Arbeitskräften geordnet und in die drei Abschnitte Vorbereitung, Untersuchung und Nachbereitung unterteilt.

Tabelle 4-4: Zeitmessung Arzt

\begin{tabular}{|l|l|}
\hline & Zeitmessung Arzt \\
\hline I & Vorbereitung \\
\hline $1 \mathrm{a}$ & Begutachtung der Anforderung \\
\hline $1 \mathrm{~b}$ & Gespräch mit Patienten und Aufklärung \\
\hline $1 \mathrm{c}$ & Festlegen der Untersuchungsstrategie \\
\hline $1 \mathrm{~d}$ & Kurzbefund letzter Patient \\
\hline II & Untersuchung \\
\hline $1 \mathrm{e}$ & Zugang legen evtl. Prämedikation \\
\hline $1 \mathrm{f}$ & Überprüfung der Einstellung \\
\hline $1 \mathrm{~g}$ & Untersuchung am Monitor beobachten \\
\hline $1 \mathrm{~h}$ & $\begin{array}{l}\text { Kontrastmittel-Einfluss im } \\
\text { Untersuchungsraum kontrollieren }\end{array}$ \\
\hline $\mathrm{Ii}$ & Untersuchung am Monitor beobachten \\
\hline $1 \mathrm{j}$ & $\begin{array}{l}\text { Eventuell Gespräch mit Patienten im } \\
\text { Untersuchungsraum }\end{array}$ \\
\hline III & Nachbereitung \\
\hline $1 \mathrm{k}$ & Bilder begutachten am Leuchtkasten \\
\hline II & $\begin{array}{l}\text { Eventuell weitere Einstellungen bei der } \\
\text { MTRA anfordern }\end{array}$ \\
\hline
\end{tabular}




\begin{tabular}{|l|l|}
\hline $1 \mathrm{~m}$ & Oberarzt anpiepen \\
\hline $1 \mathrm{n}$ & $\begin{array}{l}\text { Eventuell Voraufnahmen zum Vergleich } \\
\text { begutachten }\end{array}$ \\
\hline 10 & $\begin{array}{l}\text { Besprechung des Befundes mit dem } \\
\text { Oberarzt }\end{array}$ \\
\hline $1 \mathrm{p}$ & Verfassen des Befundes \\
\hline $1 \mathrm{q}$ & $\begin{array}{l}\text { Eventuell Abschlussgespräch mit dem } \\
\text { Patienten }\end{array}$ \\
\hline
\end{tabular}

Tabelle 4-5: Zeitmessung MTRA 1

\begin{tabular}{|l|l|}
\hline & Zeitmessung MTRA 2 \\
\hline I & Vorbereitung \\
\hline $2 \mathrm{~b}$ & $\begin{array}{l}\text { Anforderungen und Akten des } \\
\text { Patienten/Begrüßung }\end{array}$ \\
\hline $2 \mathrm{c}$ & $\begin{array}{l}\text { Patientendaten im Robsyssystem } \\
\text { eingeben }\end{array}$ \\
\hline $2 \mathrm{~d}$ & $\begin{array}{l}\text { Arzt zur Begutachtung der Anforderungen } \\
\text { rufen }\end{array}$ \\
\hline $2 \mathrm{e}$ & $\begin{array}{l}\text { Kreatininwert kontrollieren bzw. } \\
\text { Nachfragen }\end{array}$ \\
\hline $2 \mathrm{f}$ & Patienten den Aufklärungsbogen geben \\
\hline $\mathrm{II}$ & Vorbereitung des Untersuchungsraumes \\
\hline $2 \mathrm{~g}$ & Untersuchung \\
\hline $2 \mathrm{~h}$ & $\begin{array}{l}\text { Patienten in den Untersuchungsraum } \\
\text { hereinbitten, Untersuchungslauf erklären }\end{array}$ \\
\hline $2 \mathrm{i}$ & Arzt zur Kontrastmittel-Anlage rufen \\
\hline
\end{tabular}




\begin{tabular}{|c|c|}
\hline $2 \mathrm{j}$ & Eventuell Prämedikation vorbereiten \\
\hline $2 k$ & $\begin{array}{l}\text { Eventuell neuen Patienten abrufen, } \\
\text { eventuell telefonieren }\end{array}$ \\
\hline 21 & $\begin{array}{l}\text { Aufklärungsbogen zum Durchlesen } \\
\text { geben }\end{array}$ \\
\hline $2 m$ & $\begin{array}{l}\text { Kontrastmittel-Schlauch abklemmen, } \\
\text { Patienten vom Tisch nehmen }\end{array}$ \\
\hline $2 n$ & Zugang des Patienten ziehen \\
\hline III & Nachbereitung \\
\hline $2^{\circ}$ & $\begin{array}{l}\text { Dem Patienten die Bilder aushändigen, } \\
\text { Verabschiedung }\end{array}$ \\
\hline
\end{tabular}

Tabelle 4-6: Zeitmessung MTRA 2

\begin{tabular}{|l|l|}
\hline & Zeitmessung MTRA 2 \\
\hline Ia & Vorbereitung \\
\hline $3 \mathrm{~b}$ & $\begin{array}{l}\text { Nachbearbeitung von Patientendaten von } \\
\text { der vorherigen Untersuchung }\end{array}$ \\
\hline 3c & $\begin{array}{l}\text { Eventuell Voraufnahmen aus dem } \\
\text { Archiv/CT holen }\end{array}$ \\
\hline III & $\begin{array}{l}\text { Patiententüten kleben und beschriften, } \\
\text { Voraufnahmen }\end{array}$ \\
\hline $3 \mathrm{~d}$ & Untersuchung \\
\hline $3 \mathrm{e}$ & $\begin{array}{l}\text { Patientendaten eingeben und CT- } \\
\text { Topogramm laden }\end{array}$ \\
\hline $3 \mathrm{f}$ & Topogramm fahren, Untersuchung planen \\
\hline $3 \mathrm{~g}$ & Kontrastmittelplanung \\
\hline
\end{tabular}




\begin{tabular}{|l|l|}
\hline 3h & Kontrastmittel-Parameter einstellen \\
\hline III & Nachbereitung \\
\hline $3 \mathrm{i}$ & $\begin{array}{l}\text { Nachverarbeitung (Lungenfenster, } \\
\text { Topogramm) }\end{array}$ \\
\hline $3 \mathrm{j}$ & Arzt rufen \\
\hline $3 \mathrm{k}$ & $\begin{array}{l}\text { Eventuell erneute Nachbearbeitung der } \\
\text { Bilder }\end{array}$ \\
\hline $3 \mathrm{l}$ & $\begin{array}{l}\text { Bilder aus der Entwicklungsmaschine } \\
\text { nehmen, sortieren }\end{array}$ \\
\hline $3 \mathrm{~m}$ & Tisch und Gantry säubern \\
\hline $3 \mathrm{n}$ & Transport rufen \\
\hline 30 & U im Robsys abrechnen \\
\hline $3 \mathrm{p}$ & X Faktor: \\
\hline
\end{tabular}

Phase II - Messdatenerfassung

An den Arbeitsplätzen wurden mit Hilfe einer Stoppuhr jeder abgelaufene Vorgang gemessen. Der Beginn und das Ende jedes einzelnen Vorganges wurden notiert und aus der Differenz die Dauer berechnet. Zusätzlich wurden besondere Vorfälle vermerkt. Die gesammelten Daten wurden in einer Tabellenkalkulation (Microsoft Excel 97/2000, Microsoft) erfasst. Die Daten wurden anschließend in die einzelnen Untersuchungs- und Patientenkategorien eingeteilt und getrennt voneinander weiterverarbeitet.

Anfertigung des Netzplanes

1. Auftretenshäufigkeit

2. Schätzung der Vorgangs- und Verknüpfungsdauern (Wahrscheinlichkeitsverteilung)

3. Berechnung der frühesten und spätesten Zeitpunkte

4. Bestimmung der Zeitreserven und Optimierung des Netzplanes

5. Auftretenshäufigkeit 
Die Auftretenshäufigkeit $(n(V))$ der einzelnen Vorgänge wurde ermittelt. Zur weiteren Berechnung der realistischen Ablaufzeit wurden nur Vorgänge verwendet, die häufiger als $25 \%$ während den gemessenen Untersuchungen durchgeführt wurden.

\section{Stochastische Zeitplanung}

Viele Arbeitsabläufe sind bezüglich der Dauer ihrer Einzelvorgänge nicht exakt berechenbar. Vor allem längerfristige Vorhaben, erst- oder einmalige Vorgänge bereiten Schwierigkeiten bei der Ermittlung sicherer Zeitwerte der Vorgangsdauer D. Für den Fall, dass die voraussichtliche Dauer der Vorgänge geschätzt werden muss, ist das Lösungsverfahren PERT entwickelt worden, welches hinsichtlich der logischen Struktur von gleichen Annahmen wie CPM ausgeht, jedoch die Unsicherheiten hinsichtlich der Vorgangsdauer D durch Schätzung von drei Zeitwerten erfasst (Abbildung 4-16):

1. Optimistische Dauer OD

2. Häufigste Dauer HD

3. Pessimistische Dauer PD 
- Anwen den der CPM/PERT Methoden

- Errechnung der Pufferzeiten, der gesamten Projektdauer, der op timistisch en, p essim istischen und realistischen Projektzeiten
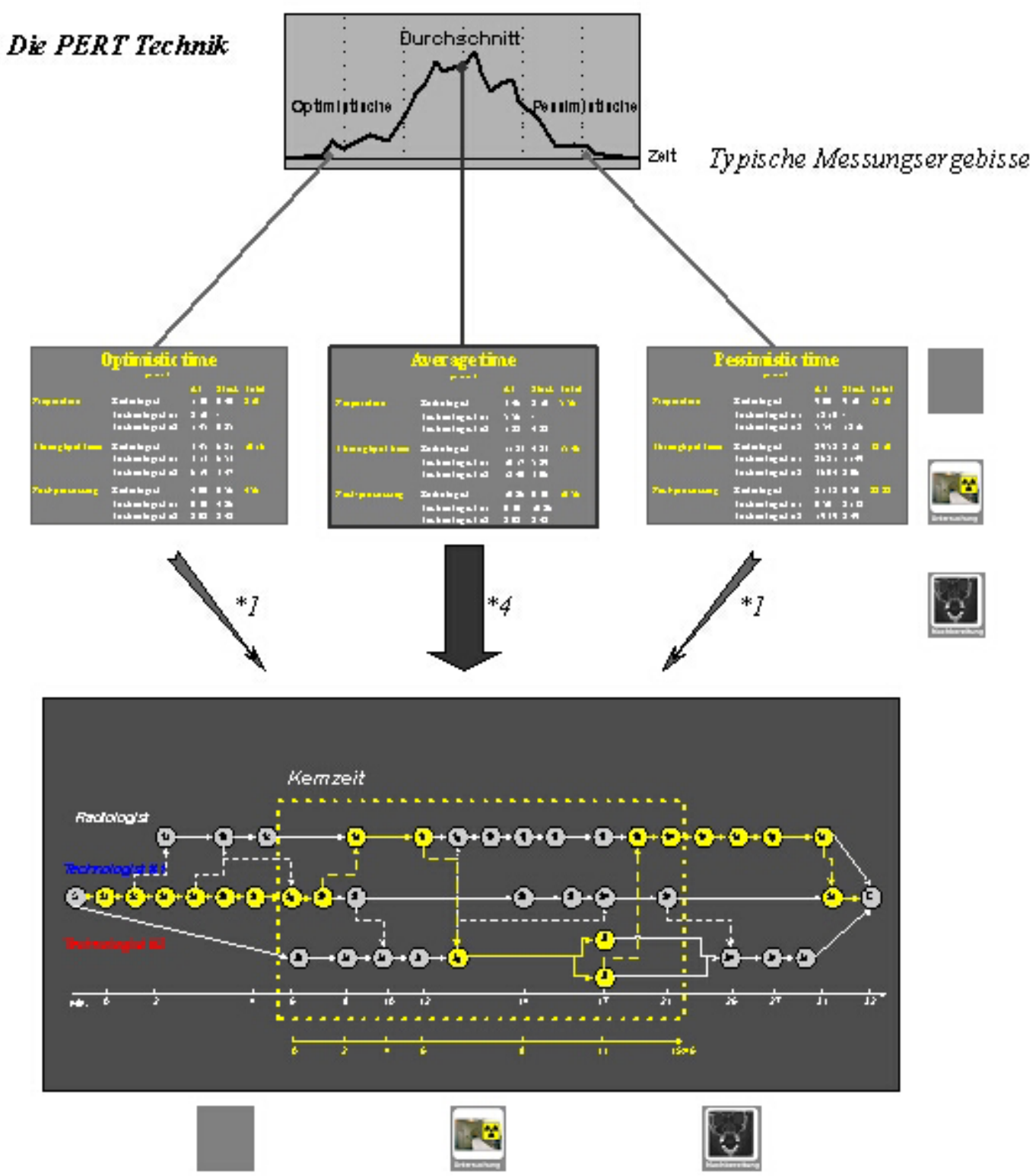

Abbildung 4-16: Schematische Darstellung der Erstellung von PERT Diagrammen aus den Zeitmessungen.

Innerhalb des Intervalls [OD, PD] wird unter Annahme einer Beta-Verteilung der Erwartungswert der realistischen Dauer RD und die zugehörige Varianz VD berechnet. Die Varianzen der Wahrscheinlichkeitsverteilung dienen als Streuungsmaß. Um den Prozessplan zu entwickeln wurde die realistische Zeit für die Dauer der einzelnen Vorgänge verwandt (RD). Der Durchschnitt des zeitlichen Beginns jedes Vorganges wurde berechnet und mit der realistischen Dauer in einem vorläufigen Zeitplan zusammengeführt. Darauf basierend wurde ein Überblick über 
die tatsächlich abgelaufene Vorgangsfolge geschaffen. Bestimmung der zeitlichen Abfolge: Alle Aktivitäten erhielten definierte Vorgänger und wurden zueinander in Abhängigkeit gebracht. Kriterien waren die deterministische Reifenfolge und die zur Verfügung stehenden Humanressourcen. Die Vorgangsbeziehungen sind Kapitel 3.2. zu entnehmen.

Phase III - Bestimmung der Zeitreserven und Optimierung des Netzplanes

Dabei wurden die Vorgänge in die Abschnitte Vorbereitung, Untersuchung und Nachbereitung gegliedert. Die Dauer der Abschnitte diente einerseits zur Validierung gegenüber den erhobenen Messdaten. Andererseits konnte sie zum Vergleich zwischen den Patientenkategorien und Maschinenkategorien herangezogen werden. Zusammenfassend wurden Vorschläge zur Optimierung des gesamten Prozesses erarbeitet und vorgestellt.

\subsubsection{Auswertung}

Um eine ausreichend hohe Zahl an vergleichbaren Untersuchungen zu gewährleisten, wurde die Auswertung auf folgende Gruppen begrenzt:

1. Die Personen-Gruppe - CT S, mobile Patienten mit Thoraxuntersuchung zur Beurteilung der eingesetzten Arbeitskräfte und ihrer Zusammenarbeit durch Analyse der Einzelschritte.

2. Die Patienten-Gruppe - CT $S$, alle Patientenkategorien mit Thoraxuntersuchungen - zum Vergleich der Abschnittsdauern mit dem Schwerpunkt auf die Durchführung der Untersuchung.

3. Die Maschinen-Gruppe - Kopfuntersuchungen aller Patientenkategorien an beiden Geräten - zum Vergleich des Einflusses der Maschinen auf den Gesamtablauf.

\subsubsection{Ergebnisse}

\subsubsection{Messdatenerfassung}

Patientenkategorien

Tabelle 4-7: Untersuchungszahlen an den CT-Geräten unterteilt nach Untersuchungsarten für das Jahr 1999

Zeitraum: 1.1.1999-

31.12.1999
Anzahl Untersuchungen
Anzahl Patienten

\section{CT S Gesamtanzahl}

7186

4469 


\begin{tabular}{|c|c|c|c|}
\hline & CT Kopf gesamt & $2276(31,7 \%)$ & $1761(39,4 \%)$ \\
\hline & CTK (Kopf) & 580 & \\
\hline & CTKK (Kopf mit KM) & 140 & \\
\hline & CTKO (Kopf ohne KM) & 1556 & \\
\hline & CT Thorax gesamt & $1227(17,1 \%)$ & $983(22,0 \%)$ \\
\hline & CTT (Thorax) & 136 & \\
\hline & CTTK (Thorax mit KM) & 193 & \\
\hline & CTTO (Thorax ohne KM) & 898 & \\
\hline \multirow[t]{9}{*}{ СТ 4} & Gesamtanzahl & 10674 & 5078 \\
\hline & CT Kopf gesamt & $2029(19,0 \%)$ & $1614(31,8 \%)$ \\
\hline & CTK (Kopf) & 500 & \\
\hline & CTKK (Kopf mit KM) & 91 & \\
\hline & CTKO (Kopf ohne KM) & 1438 & \\
\hline & CT Thorax gesamt & $1514(14,2 \%)$ & $1206(23,7 \%)$ \\
\hline & CTT (Thorax) & 203 & \\
\hline & CTTK (Thorax mit KM) & 1023 & \\
\hline & CTTO (Thorax ohne KM) & 288 & \\
\hline
\end{tabular}

Die chronlogische Reihenfolge der Untersuchungen ist in Tabelle 4-4 - 4-6 getrennt für die MTRAs und Ärzte zusammengefasst.

\subsubsection{Die CT-Untersuchung wurde in drei Abschritten getrennt betrachtet:}

Vorbereitung

Der erste Abschnitt der Untersuchung war die Vorbereitung der CT-Diagnostik. Die Untersuchung begann mit dem Bereitlegen der Anforderungen und Akten des Patienten und der Begrüßung (2a). Anschließend wurde der Radiologe zur Begutachtung der Anforderungen gerufen (2c). Unterdessen gab die MTRA 1 die Patientendaten im RIS (Robsys) ein (2b), kontrollierte den Kreatininwert, der unter Umständen telefonisch auf der Station oder im Labor nachgefragt werden musste (2d), und gab dem Patienten den Aufklärungsbogen (2e). Unterdessen hatte der Radiologe die Anforderung begutachtet (1a) und widmete sich anschließend der Aufklärung des Patienten (1b). In diesen Zeitraum fiel für die MTRA 
2 die Nachbearbeitung von Patientendaten von der vorherigen Untersuchung (3a), die Vorbereitung einer neuen Patiententüte durch Kleben und Beschriften (3b) und das Beschaffen der Voraufnahmen durch einen möglicherweise notwendigen Gang in das

Bildarchiv

an.

Nach der Vorbereitung des Untersuchungsraumes durch die MTRA 1 (2f) fing der nächste große Abschnitt, die Untersuchung (Durchführungszeitraum), an (Tabelle 4$4)$.

\section{Untersuchung}

Die Zeitdauer der Untersuchung wurde als die Zeit definiert, während der sich der Patient im CT-Untersuchungsraum aufhielt. Dieser Abschnitt begann mit dem Vorgang 2g der MTRA 1, den Patienten in den Untersuchungsraum herein zu bitten und den Untersuchungsablauf zu erklären. Währenddessen legte der Radiologe die Untersuchungsstrategie fest (1c). Falls zwischendurch Zeit verblieb, beschäftigte sich der Radiologe mit der Verfassung des Kurzbefundes des letzten Patienten (1d). Daraufhin rief die MTRA 1 den Arzt zur Kontrastmittelanlage (2h), der dieser Aufforderung mit der Tätigkeit des Zuganglegens und möglicher Verabreichung von Prämedikation nachkam (1e). Währenddessen gab die zweite MTRA die Patientendaten für den Computertomographen ein und lud das benötigte CTTopogramm (3d). Der Patient wurde zum Topogramm von der MTRA 1 eingestellt (2i), eventuell gefolgt von der Vorbereitung der Prämedikation (2j). Anschließend resultierte das Fahren des Topogramms mit Planung der Untersuchung (3e) und der Kontrastmittelapplikation (3f) durch die MTRA 2. Vor der eigentlichen Untersuchung des Patienten überprüfte der Radiologe die Einstellungen (1f). Der zentrale Vorgang, die Durchführung der Untersuchung (3g), wurde vom Radiologen am Monitor verfolgt (1g). Nach Ablauf der Nativserie erfolgte die Einstellung der Kontrastmittelparameter durch die MTRA 2 (3h) und die Kontrolle des Kontrastmitteleinflusses im Untersuchungsraum durch den Radiologen (1h). Erneut verfolgte der Radiologe die Untersuchung der Kontrastmittelserie am Bildschirm (1i). Zu jeder Zeit war ein erwünschtes Gespräch mit dem Patienten im Untersuchungsraum (1j) möglich. Während der Untersuchung organisierte die MTRA 1 die folgenden Untersuchungen durch Abruf, eventuell telefonisch (2k)., Dem nachfolgenden Patienten wurde der
Aufklärungsbogen
zum
Durchlesen
ausgehändigt
(2I).

Nach Abschluss der apparativen Untersuchung wurde der Kontrastmittelschlauch 
abgeklemmt und der Patient von der Patientenliege genommen (2m). Falls nicht noch der Zugang des Patienten von der MTRA 1 gezogen wurde (2n), endete damit der Abschnitt Untersuchung. Unterdessen lief die Nachbereitung der Untersuchung durch Nachbearbeitung der Bilddaten an (3i). Die ersten Bilder wurden zum Ausdruck geschickt, die Bilder aus der Entwicklungsmaschine genommen, sortiert und am Lichtkasten oder dem Alternator aufgehängt (3l). Nach Ruf des Radiologen (3j) konnte dieser mit der Bilddiagnostik an Leuchtkasten oder Alternator beginnen (1k)_(Tabelle 4-4).

\section{Nachbereitung}

Nach Anforderung des Radiologen nach weiteren Bildeinstellungen bei der MTRA (11) erfolgte eine Nachbearbeitung der Bilder (3k). Unterdessen konnte zur Absprache der Diagnostik der Oberarzt gerufen werden $(1 \mathrm{~m})$. Weiterhin war in einigen Fällen die Begutachtung vorhandener Voraufnahmen zum Vergleich erforderlich

Aufgabe der MTRA war zu diesem Zeitpunkt die Säuberung des Tisches und der Gantry (3m), außerdem bei Bedarf der Anruf des Rücktransportes zur Station (3n) und die Abrechnung der Untersuchung im RIS (30). Bei Bedarf wurden die Befunde mit dem Oberarzt (10) besprochen. Der Radiologe verfasste abschließend den Befund (1p). Ein Abschlussgespräch mit dem Patienten (1q) und die Aushändigung der Bilder mit Verabschiedung (2o) rundeten den Prozess ab (Tabelle 4-4). 


\subsubsection{Erhobene Messdatenmenge}

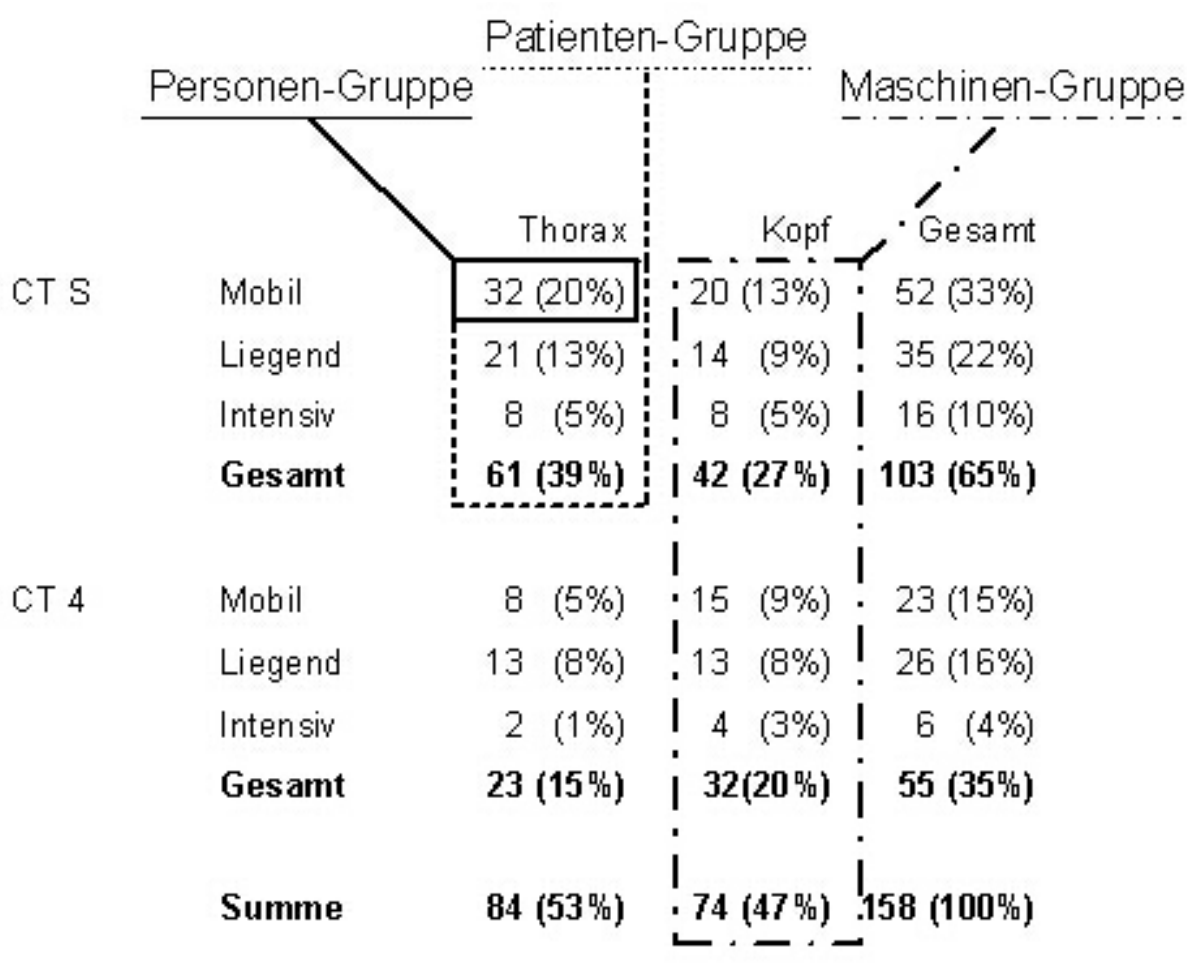

Abbildung 4-17: Verteilung der erfassten Untersuchungen

Die Abbildung zeigt die Aufschlüsselung der erfassten Untersuchung. Es wurden $\begin{array}{lllll}\text { Daten } & \mathrm{zu} & 158 & \text { Untersuchungen }\end{array}$ Die Phase II, die Messdatenerfassung begann mit Einzelmessungen. Pro Untersuchung wurden zwischen 13 und 41 Einzelzeitmessungen aufgezeichnet. Dabei konnten die Vorgänge mit Ihrer absoluten Anfangszeit sowie der Zeitdauer einzeln erfasst werden. Somit konnte deren Auftretenshäufigkeit ermittelt werden. Zur Berechnung der Daten wurden im folgenden die Ergebnisse der Thoraxuntersuchungen von mobilen Patienten am CT S (Personen-Gruppe) exemplarisch für das Erstellen eines Prozessmodells verwendet.

\subsubsection{Wahrscheinlichkeitsverteilung}

Bei den optimistischen Zeitdauern der Vorgänge beim Radiologen konnten meist Zeiten unter einer Minute gemessen werden. Bei den pessimistischen Zeiten waren in der Vorbereitungszeit die Aufklärung des Patienten mit 7:00 Minuten und das Legen des Zugangs mit 8:15 Minuten (Vorgänge $1 \mathrm{~b}$ und $1 \mathrm{e}$ ) verlängert. Ebenfalls längere pessimistische Arbeitszeiten stellten sich während des Bilderbegutachtens mit 14:20 Minuten und dem meist gleichzeitig stattfindenden Befunden mit 14:00 
Minuten ein (Vorgänge $1 \mathrm{k}$ und $1 \mathrm{p}$ in Tabelle 4-4). Sämtliche Vorgänge der MTRA 1 konnten mit einer optimistischen Zeit unter einer Minute festgehalten werden. Realistisch zeigten sich dort längere Zeiten, wenn die Varianz VAR (D) auch erhöht war. Mit einer Varianz von 9:31 Minuten zeigte sich dies besonders in dem Arbeitsschritt 3l - Bilder aus der Entwicklungsmaschine nehmen und sortieren. Diese Verzögerungen kamen durch die teilweise großen Zeitabstände zwischen erstem und letztem Ausdruck zustande. Bei den Arbeitsvorgängen der MTRA 2 war die Durchführung der Untersuchung von großer Varianz. Zeigten die Messungen in den häufigsten Fällen Zeiten zwischen 1 Minute und 8 Minuten, so dauerten 5 Untersuchungen zwischen 14 und 22 Minuten. Im pessimistischen Fall wurde eine Zeit von 32:19 Minuten gemessen. In den meisten Fällen waren erweiterte Untersuchungen durch Wiederholungen oder Protokollabweichungen die Ursache für lange Verzögerungen. Technische Probleme waren während einer Untersuchung der Grund für eine Verlängerung der Untersuchungszeit.

Ein weiterer variabler Vorgang war das Nachverarbeiten des Lungenfensters, das sich auch nachhaltig auf das Drucken und anschließende Befunden auswirkte. Dabei wurde eine Varianz von 5:59 Minuten gemessen. 


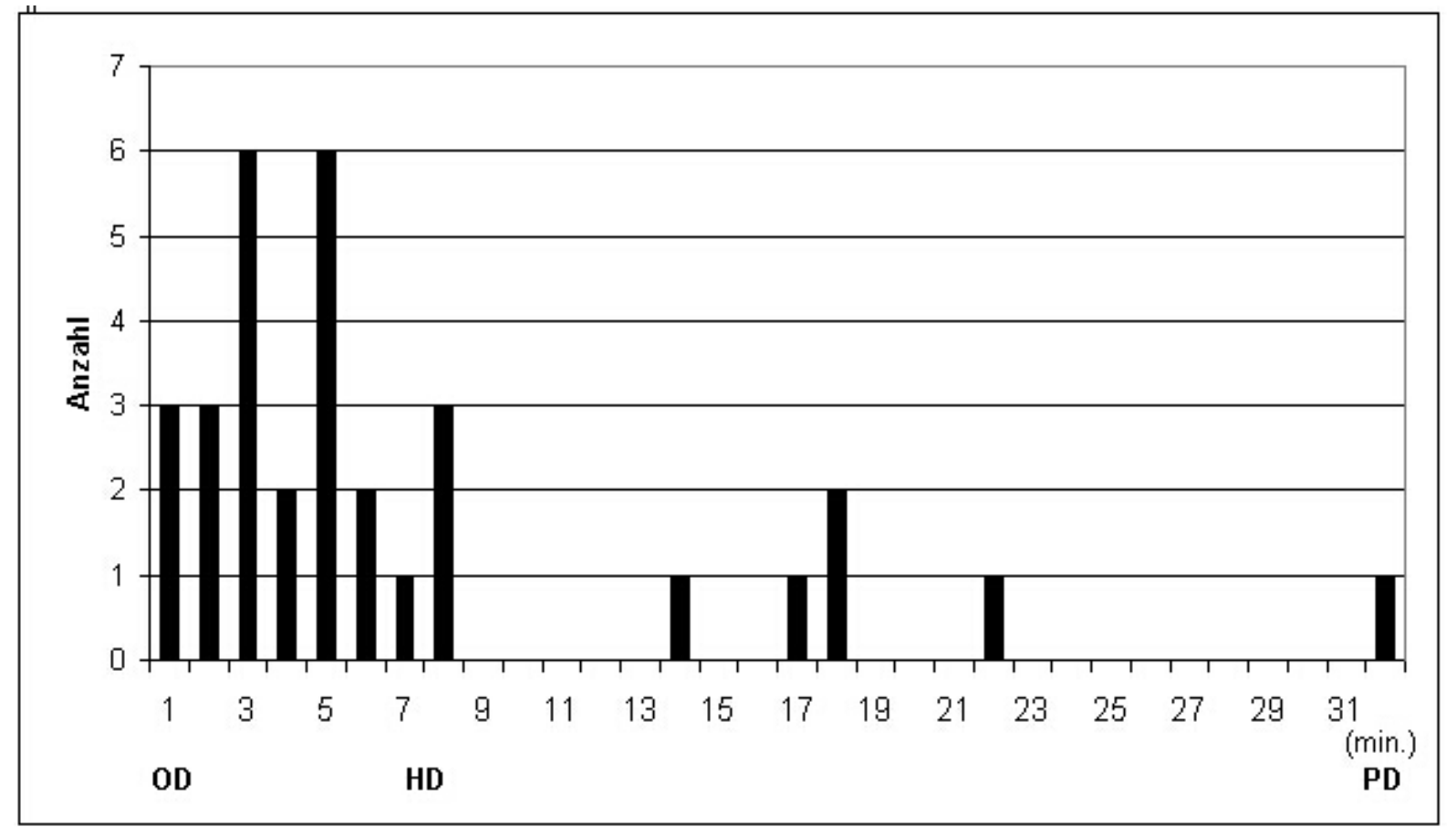

Abbildung 4-18: Vorgang $3 g$ - Untersuchung durchführen (CT S, CT Thorax, mobile Patienten: $n=32$ ). Anzahl der gemessenen Zeitdauern. OD: Optimistische Dauer; HD: Häufigste Dauer; PD: Pessimistische Dauer. Werte in Minuten.

Tabelle 4-8: Vorgänge des Radiologen: PERT (CT S, CT Thorax, mobile Patienten: $n=32)$. $n(V)$ : Auftretenshäufigkeit der Vorgänge; RD: realistische Dauer; OD: optimistische Dauer; PD: pessimistische Dauer.

\begin{tabular}{|c|c|}
\hline$\overline{\text { Nr.Vorgang }}$ & $\overline{n(V)}$ RD OD PD VAR(D) \\
\hline Vorbereitung & \\
\hline 1a Begutachtung der Anforderung & 22 00:3300:1001:18 0,04 \\
\hline 1b Gespräch mit Patienten und Aufklärung & 30 03:0600:3007:00 1,17 \\
\hline II Untersuchung & \\
\hline 1c Festlegen der Untersuchungsstrategie & 17 00:3000:0501:00 0,02 \\
\hline 1e Zugang legen, evtl. Prämedikation & 31 03:4001:1008:15 1,39 \\
\hline 1f Überprüfung der Einstellung & 27 00:3000:0501:14 0,04 \\
\hline 1g Untersuchung am Monitor beobachten & 12 00:5600:1002:00 0,09 \\
\hline 1h Kontrastmittel-Einfluss im Untersuchungsraum kontrolliere & $2600: 5500: 0402: 50 \quad 0,21$ \\
\hline 1i Untersuchung am Monitor beobachten & 22 02:1800:1007:00 1,30 \\
\hline 1j Evtl. Gespräch mit Patienten im Untersuchungsraum & 11 02:0200:1005:00 0,65 \\
\hline III Nachbereitung & \\
\hline 1k Bilder am Leuchtkasten begutachten & 26 05:2300:5014:20 5,06 \\
\hline
\end{tabular}




\begin{tabular}{lccc}
\hline 11 Evtl. weitere Einstellungen bei der MTRA anfordern & 1 & $01: 3901: 3901: 39$ & 0,00 \\
\hline 1mOberarzt anpiepen & 4 & $00: 4300: 1001: 20$ & 0,04 \\
\hline 1n Evtl. Voraufnahmen zum Vergleich begutachten & 3 & $02: 3001: 5903: 00$ & 0,03 \\
\hline 1o Besprechung des Befundes mit dem Oberarzt & 6 & $02: 3801: 0005: 00$ & 0,44 \\
\hline 1p Verfassen des Befundes & 20 & $06: 0302: 0014: 00$ & 4,00 \\
\hline 1q Evtl. Abschlussgespräch mit dem Patienten & 3 & $00: 4200: 3001: 00$ & 0,01 \\
\hline
\end{tabular}

Tabelle 4-9: Vorgänge der MTRA 1: PERT (CT S, CT Thorax, mobile Patienten: $\mathrm{n}=32)$. $\mathrm{n}(\mathrm{V})$ : Auftretenshäufigkeit der Vorgänge; RD: realistische Dauer; OD: optimistische Dauer; PD: pessimistische Dauer. Zeiten in Minuten.

\section{Nr.Vorgang}

I Vorbereitung

2a Anforderungen und Akten des Pat./Begrüßung

2c Arzt zur Begutachtung der Anforderungen rufen

2d Kreatininwert kontrollieren bzw. nachfragen

2e Pat. Den Aufklärungsbogen geben

\section{Untersuchung}

2f Vorbereitung des Untersuchungsraumes

2g Pat. in den Untersuchungsraum bitten, Ablauf erklären

2h Arzt zur KM-Anlage rufen

2i Pat. Zum Topogramm einstellen

2j Evtl. Prämedikation vorbereiten

$2 \mathrm{k}$ Evtl. neuen Pat. abrufen, evtl. telefonieren

21 Aufklärungsbogen zum Durchlesen geben

2mKM-Schlauch abklemmen, Pat. vom Tisch nehmen

$3 m$ Tisch und Gantry säubern

3o U im Robsys abrechnen

\section{Nachbereitung}

31 Bilder aus der Entwicklungsmaschine nehmen, sortieren 31 07:5000:3019:00 9,51

20 Pat. die Bilder aushändigen, Verabschiedung

14 00:2900:1000:50 $\quad 0,01$

\section{$n(V)$ RD OD PD VAR(D)}

27 00:4200:0502:21 0,14

4 00:1900:0700:40 $\quad 0,01$

16 00:4300:0702:10 $\quad 0,12$

20 00:3400:1001:30 0,05
29 03:0800:1010:00 2,69

32 02:0800:0505:40 $\quad 0,87$

11 00:3300:0401:20 0,04

17 00:5900:1502:00 0,09

6 01:5400:2204:40 0,51

17 01:3300:0404:00 0,43

5 00:4300:2901:00 0,01

31 03:2200:4106:00 0,79

31 01:3400:0504:00 0,43

32 03:0800:2209:40 2,40 
Tabelle 4-10: Vorgänge der MTRA 2: PERT (CT S, CT Thorax, mobile Patienten: $n=32)$. $n(V)$ : Auftretenshäufigkeit der Vorgänge; RD: realistische Dauer; OD: optimistische Dauer; PD: pessimistische Dauer, Zeiten in Minuten).

Nr.Vorgang

$n(V)$ RD OD PD VAR(D)

I Vorbereitung

3b Patiententüten kleben u. beschriften, Voraufnahmen 28 01:3500:10 03:20 0,28

\begin{tabular}{llll}
\hline 3c Evtl. Voraufnahmen aus dem Archiv/CT holen $\quad 2$ & $02: 4001: 0004: 40$ & 0,37
\end{tabular}

\section{Untersuchung}

2b Patientendaten im Kliniksystem (Robsys) eingeben 20 01:2000:07 03:20 0,29

3d Patientendaten eingeben und CT-Topogramm laden 32 01:5100:12 05:10 0,69

\begin{tabular}{llc}
\hline 3e Topogramm fahren, Untersuchung planen & 32 01:4700:1803:50 & 0,35 \\
\hline 3f Kontrastmittelplanung & 26 00:5300:10 02:50 & 0,20 \\
\hline 3g Untersuchung durchführen & 32 10:3001:00 32:19 & 27,24 \\
\hline 3i Nachverarbeitung (Lungenfenster, Topogramm) & 30 07:3900:30 15:10 & 5,98 \\
\hline
\end{tabular}

III Nachbereitung

\begin{tabular}{|c|c|c|}
\hline 3k Evtl. erneute Nachbearbeitung der Bilder & 8 03:2600:30 08:00 & 1,56 \\
\hline 2n Zugang des Patienten ziehen & 11 01:1400:10 03:10 & 0,25 \\
\hline 3n Transport rufen & 7 00:2300:05 00:40 & 0,01 \\
\hline $3 p \times$ Faktor & 5 02:1102:00 03:00 & 0,03 \\
\hline
\end{tabular}

Die durch PERT gewonnenen realistischen Vorgangsdauern wurden nun in einem ersten Schritt auf den Netzplan gebracht. Um die zeitliche Reihenfolge der einzelnen Arbeitsschritte zu berücksichtigen, wurden die Anfangszeiten jedes Vorgangs zur Orientierung im Durchschnitt verwendet. Dabei zeigte sich bei den Vorgängen $1 \mathrm{k}$ und $1 p$ - Bilder am Leuchtkasten begutachten und Verfassen des Befundes - eine Gleichzeitigkeit, die berücksichtigt werden musste. Ein weiteres Beispiel waren der Vorgang 3h - KM-Parameter einstellen - , der während des Vorganges $3 g$ Durchführung der Untersuchung - stattfand. In diesen Fällen wurde die AnfangAnfang-Beziehung verwendet.

Es wurden Vorgänge identifiziert, die bisher fest einer Arbeitskraft zugeordnet wurden, sich aber im gesamten Verlauf als kritisch erwiesen. Soweit möglich wurden daher Vorgänge von derjenigen Arbeitskraft übernommen, die dafür ausreichend Pufferzeiten zur Verfügung hatte. Dies wurde bei den durchgeführten Messungen in 
23 Fällen der 32 Messungen (71,9\%) beobachtet.

Die zusätzlichen Vorgänge $1 \mathrm{r}$ und 1s waren Telefonate des Radiologen $(9,3 \%)$, Legen und Spülen des Zugangs (6,2\%) und ein Aufklärungsgespräch (3,1\%). Bei der MTRA 1 waren ebenso Telefonate zusätzlich in 12,5\%, Transporttätigkeiten in 12,5\% und versorgende Aufgaben in 6,2\% der Fälle zu verzeichnen (Vorgänge 2p und 2q). Die MTRA 2 war neben den regulären Vorgängen in 6,2\% mit Terminplanungen und in 3,1\% der Messungen mit einem Patientengespräch beschäftigt (Vorgänge 3q und 3r). Der X-Faktor (Vorgang 3p) und damit der technische Versorgungsfaktor war mit 5 von 32 Untersuchungen selten zu beobachten (15,6\%). Diese Vorgänge wurden im fortschreitenden Erstellen des Netzplans nicht berücksichtigt.

\subsubsection{Bestimmung der Zeitreserven und Optimierung des Netzplanes}

\section{Kritische Vorgänge}

Die kritischen Vorgänge während der Vorbereitung der Untersuchung waren sowohl bei der MTRA 1 als auch dem Radiologen zu erwarten. Dabei stand die Aufklärung des Patienten über die Gabe von Kontrastmittel sowie die Vorbereitung der Kontrastmittelgabe im Vordergrund (Vorgänge 2e, 1b, 1e). Während der Untersuchungszeit spielten die Arbeitsschritte zur richtigen Auswahl der Untersuchungseinstellung am Gerät eine kritische Rolle. Die Bedienung des Gerätes durch die MTRA 2 und die Kontrolle der Einstellungen durch den Radiologen lagen dabei auf dem kritischen Weg (Vorgänge 1c, 3d, 1f, 3f, 3e, 3g, 3h). Nach der durchgeführten Untersuchung waren die Vorgänge im Untersuchungsraum als kritisch errechnet worden. Dazu zählte das Freimachen und anschließende Säubern des Untersuchungstisches durch die MTRA 1 (Vorgänge 2m, 3m). Der letzte kritische Vorgang zum Abschluss der Untersuchung war die Abrechnung im RIS

Der kritische Weg während der Nachbereitung wurde durch die parallel stattfindenden Vorgänge des Bilder zur Verfügung stellen und gleichzeitigen Befundens (Vorgang 3l, 1k, 1p) aufgezeigt. Der letzte Vorgang war die Verabschiedung des Patienten durch die MTRA 1 (Vorgang 20). 


\subsubsection{Pufferzeiten}

Geringe Pufferzeiten wurden während der Vorbereitung bei den Vorgängen 1a des Radiologen und 2d bzw. 2h der MTRA 1 errechnet. Der Vorgang der MTRA 2 hatte eine Gesamtpufferzeit von 6:00 Minuten zu verzeichnen. Die Untersuchungszeit war für den Radiologen über die kritischen Vorgänge $1 \mathrm{c}$ und $1 \mathrm{f}$ hinaus mit sehr langen Gesamtpufferzeiten von 14:10 Minuten nicht kritisch. Die Nachbereitung war mit einer gesamten Pufferzeit von 1:47 Minuten als vorrangige Tätigkeit anzusehen. Unmittelbar um die Untersuchung arbeitet die MTRA 2 ohne Pufferzeit (Vorgänge 3e - 3g), während danach eine Pufferzeit von 4:04 Minuten errechnet wurde. Anhand der Berechnungen im Netzplan zeigte sich für die MTRA 1 eine Pufferzeit von 11:15 Minuten während der unmittelbaren Untersuchung (Vorgang $3 \mathrm{~g}$ ). Die darauffolgenden Arbeitsschritte lagen alle auf dem kritischen Weg. 


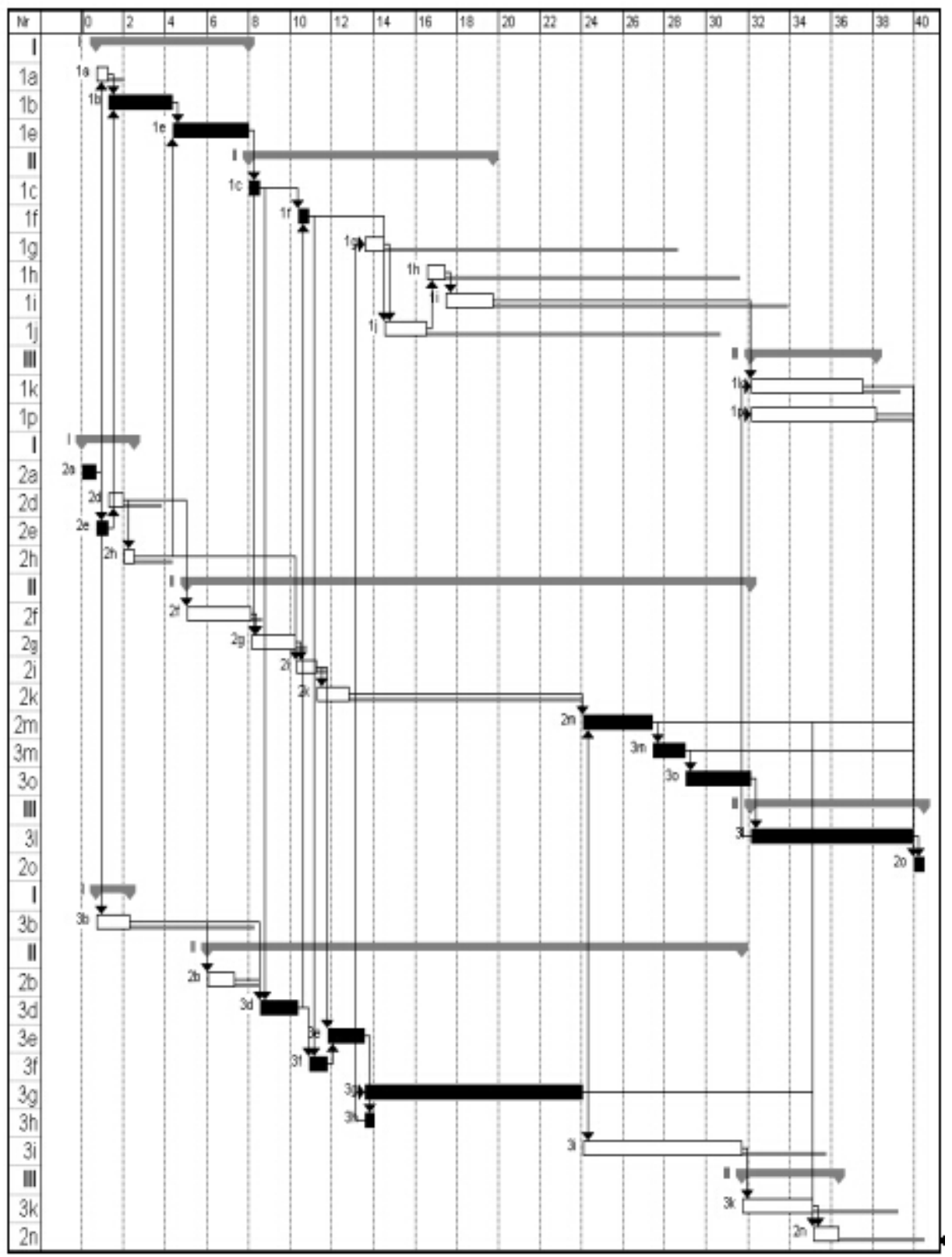

Abbildung 4-19: Realistischer Netzplan am CT S, CT Thorax, mobile Patienten. Kritische Vorgänge in schwarz; Abschnitte in grau (I: Vorbereitung; II: Durchführung; III: Nachbereitung); Nicht kritische Vorgänge in weiß; Gesamte Pufferzeit nach nichtkritischen Vorgängen in grau. Zeit in Minuten. 


\subsubsection{Auswertung}

Abschnitt der Untersuchung

In den Untersuchungsabschnitt (Kernzeit) wurden diejenigen Vorgänge in den Netzplan aufgenommen, die zwingend notwendig für den Ablauf der Untersuchung im Untersuchungsraum sind. Dies wurde in diesem Vorgangsplan umrahmt durch die Vorgänge $2 f$ - Vorbereitung des Untersuchungsraumes - und 30 - Abrechnung der Untersuchung im RIS. Alle weiteren Vorgänge wurden den Abschnitten Vorbereitung und Nachbereitung zugeordnet.

Tabelle 4-11: Vorgänge des Radiologen; CPM (CT S, CT Thorax, mobile Patienten). FAZ: Frühester Anfangszeitpunkt; SAZ: Spätester Anfangszeitpunkt; FEZ: Frühester Endzeitpunkt; SEZ: Spätester Endzeitpunkt; FP: Freie Pufferzeit; GP: Gesamte Pufferzeit. Zeiten in Minuten.

\section{Nr. Vorgang}

n: FAZ SAZ FEZ SEZ FP GP

32

\section{Vorbereitung}

1a Begutachtung der Anforderung

22 00:4 01:2 01:1 02:0 00:0 00:4 $\begin{array}{llllll}2 & 8 & 5 & 1 & 1 & 6\end{array}$

1b Gespräch mit Patienten und Aufklärung

30 01:1 01:1 04:2 04:2 00:0 00:0

1e Zugang legen, evtl. Prämedikation

31 04:2 04:2 08:0 08:0 00:0 00:0

$\begin{array}{llllll}2 & 2 & 2 & 2 & 0 & 0\end{array}$

\section{Untersuchung}

1c Festlegen der Untersuchungsstrategie

17 08:0 08:0 08:3 08:3 00:0 00:0

\begin{tabular}{|c|c|c|c|c|c|c|}
\hline 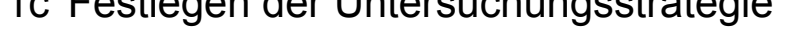 & 2 & 2 & 2 & 2 & 0 & 0 \\
\hline 1f Überprüfung der Einstellung & $27 \begin{array}{l}10: 2 \\
3\end{array}$ & $\begin{array}{l}10: 2 \\
3\end{array}$ & $\begin{array}{l}10: 5 \\
3\end{array}$ & $\begin{array}{l}10: 5 \\
3\end{array}$ & $\begin{array}{l}00: 0 \\
0\end{array}$ & $\begin{array}{l}00: 0 \\
0\end{array}$ \\
\hline 1g Untersuchung am Monitor beobachten & $12{ }_{3}^{13: 3}$ & $\begin{array}{l}27: 4 \\
3\end{array}$ & $\begin{array}{l}14: 2 \\
9\end{array}$ & $\begin{array}{l}28: 3 \\
9\end{array}$ & $\begin{array}{l}00: 0 \\
0\end{array}$ & $\begin{array}{l}14: 1 \\
0\end{array}$ \\
\hline $\begin{array}{l}\text { 1h KM-Einfluss im Untersuchungsraum } \\
\text { kontrollieren }\end{array}$ & $26{ }_{1}^{16: 3}$ & $\begin{array}{l}30: 4 \\
1\end{array}$ & $\begin{array}{l}17: 2 \\
6\end{array}$ & $\begin{array}{l}31: 3 \\
6\end{array}$ & $\begin{array}{l}00: 0 \\
0\end{array}$ & $\begin{array}{l}14: 1 \\
0\end{array}$ \\
\hline 1i Untersuchung am Monitor beobachten & $22 \begin{array}{l}17: 2 \\
6\end{array}$ & $\begin{array}{l}31: 3 \\
6\end{array}$ & $\begin{array}{l}19: 4 \\
4\end{array}$ & $\begin{array}{l}33: 5 \\
4\end{array}$ & $\begin{array}{l}12: 2 \\
3\end{array}$ & $\begin{array}{l}14: 1 \\
0\end{array}$ \\
\hline $\begin{array}{l}\text { 1j Gespräch mit Patienten im } \\
\text { Untersuchungsraum }\end{array}$ & $11{ }_{9}^{14: 2}$ & $\begin{array}{l}28: 3 \\
9\end{array}$ & $\begin{array}{l}16: 3 \\
1\end{array}$ & $\begin{array}{l}30: 4 \\
1\end{array}$ & $\begin{array}{l}00: 0 \\
0\end{array}$ & $\begin{array}{l}14: 1 \\
0\end{array}$ \\
\hline
\end{tabular}


III Nachbereitung

1k Bilder am Leuchtkasten begutachten

$26 \begin{array}{llllll}32: 0 & 33: 5 & 37: 3 & 39: 1 & 00: 0 & 01: 4\end{array}$

$1 p$ Verfassen des Befundes $20 \begin{array}{llllll}32: 0 & 33: 5 & 38: 1 & 39: 5 & 01: 4 & 01: 4\end{array}$

$\begin{array}{llllll}7 & 4 & 0 & 7 & 7 & 7\end{array}$


Tabelle 4-12: Vorgänge der MTRA 1: CPM (CT S, CT Thorax, mobile Patienten); FAZ: Frühester Anfangszeitpunkt; SAZ: Spätester Anfangszeitpunkt; FEZ: Frühester Endzeitpunkt; SEZ: Spätester Endzeitpunkt; FP: Freie Pufferzeit; GP: Gesamte Pufferzeit. Zeiten in Minuten.

I Vorbereitung

2a Anforderungen und Akten des Pat./Begrüßung 2700:0000:0000:4200:4200:0000:00

2d Kreatininwert kontrollieren bzw. nachfragen 1601:1603:0601:5903:4900:0001:50

2e Pat. den Aufklärungsbogen geben 2000:4200:4201:1601:1600:0000:00

2h Arzt zur KM-Anlage rufen 1101:5903:4902:3204:2201:5001:50

II Untersuchung

2f Vorbereitung des Untersuchungsraumes 2905:0005:3108:0808:3900:0000:31

$2 g$ Pat. in den Untersuchungsraum bitten, Ablauf erklären 3208:0808:3910:1610:4700:0000:31

2i Pat. zum Topogramm einstellen 1710:1610:4711:1511:4600:0000:31

$2 \mathrm{k}$ Neuen Pat. abrufen, evtl. telefonieren 1711:1522:3012:4824:0311:1511:15

2mKM-Schlauch abklemmen, Pat. vom Tisch nehmen 3124:0324:0327:2527:2500:0000:00

$3 m$ Tisch und Gantry säubern 3127:2527:2528:5928:5900:0000:00

3o U im Robsys abrechnen 3228:5928:5932:0732:0700:0000:00

\section{Nachbereitung}

3I Bilder aus der Entwicklungsmaschine nehmen, sortieren3132:0732:0739:5739:5700:0000:00 2o Pat. die Bilder aushändigen, Verabschiedung 1439:5739:5740:2640:2600:0000:00 
Tabelle 4-13: Vorgänge der MTRA 2: CPM (CT S, CT Thorax, mobile Patienten); FAZ: Frühester Anfangszeitpunkt; SAZ: Spätester Anfangszeitpunkt; FEZ: Frühester Endzeitpunkt; SEZ: Spätester Endzeitpunkt; FP: Freie Pufferzeit; GP: Gesamte Pufferzeit. Zeiten in Minuten.

I Vorbereitung

3b Patiententüten kleben u. beschriften, Voraufnahmen2800:4206:4202:1708:1700:4306:00

II Untersuchung

2b Patientendaten ins Kliniksystem (Robsys) eingeben 2006:0007:1207:2008:3201:1201:12

3d Patientendaten eingeben und CT-Topogramm laden3208:3208:3210:2310:2300:0000:00

3e Topogramm fahren, Untersuchung planen 3211:4611:4613:3313:3300:0000:00

3f Kontrastmittelplanung 2610:5310:5311:4611:4600:0000:00

$3 g$ Untersuchung durchführen 3213:3313:3324:0324:0300:0000:00

3h KM-Parameter einstellen 2313:3313:3314:0314:0300:0000:00

3i Nachverarbeitung (Lungenfenster, Topogramm)

3024:0328:0731:4235:4600:0004:04

\section{Nachbereitung}

3k Evtl. erneute Nachbearbeitung der Bilder

8 31:4235:4635:0839:1200:0004:04

2n Zugang des Patienten ziehen

1135:0839:1236:2240:2604:0404:04

\subsubsection{Auswertung von unterschiedlichen Kategorien innerhalb einer gleichen Untersuchungsart}

Zur Begutachtung der Patientenkategorien wurden die Ergebnisse des CT S mit der Untersuchung des Thorax untereinander verglichen. Im der bereits in Abschnitt 4.1 besprochenen Kategorie der mobilen Patienten lagen Zahlen über 32 Patienten vor. Die Anzahl der gemessenen Untersuchungen der bettlägerigen Patienten war 21, die Zeiten für Intensivpatienten wurden während 8 Untersuchungen

erhoben.

Dabei wurde der Schwerpunkt auf die realistische und optimistische Untersuchungszeit entsprechend der Durchführungszeit gelegt und für alle 3 Kategorien berechnet.

Als Ausgangswert dienten die tatsächlich beobachteten Untersuchungsdauern, diese wurden dann mit Hilfe der CPM/PERT ausgerechnet und zuletzt für verbesserte 
Diese beinhaltete das frühere Ausführen der Vorgänge 1e - i.v.-Zugang legen - und $2 \mathrm{~h}$ - Arzt zum Legen des Zugangs rufen - bereits während der Vorbereitung. Diese 3 Berechnungen wurden sowohl in der realistischen als auch in der optimistischen Zeit erfasst. Abbildung 3-6 zeigt im mobilen Patientengut (M) die realistische Durchführungszeit von 31:13 Minuten, die noch unter zusätzlicher Optimierung auf 27:07 Minuten verringert werden könnte. Ausgangswert war 36:01 Minuten. Die schnellste beobachtete Durchführungsdauer war 15:40 Minuten, die unter optimistischen Bedingungen auf 4:23 Minuten und verbessert auf 3:03 Minuten vermindert werden könnte.

Vergleichbare Werte sind in der Kategorie der bettlägerigen Patienten (L) zu erwarten. Gefunden wurde eine etwas längere Durchführungszeit von 38:30 Minuten. Nach Berechnung mit der Netzplantechnik würden 30:38 Minuten, bzw. 26:18 Minuten unter optimierten Bedingungen erreicht. Die kürzeste gemessene Durchführungszeit war 13:25 Minuten. Diese könnte im optimistischen Falle auf 3:35 Minuten und bis zu 3:20 Minuten unter optimierten Berechnungen minimiert werden. Anders sieht es dagegen in der Kategorie der Intensivpatienten (I) aus. Hier sind erwähnte Vorgänge, vor allem $2 g$, aber auch $2 m$ die entscheidend verlängernden Faktoren für die gesamte Durchführungszeit. So dauerte die Untersuchung mit dem Patienten im Untersuchungsraum 43:53 Minuten, die kürzeste Dauer war mit 29:40 Minuten noch fast doppelt so lange wie in den anderen beiden Patientenkategorien. Da bei Intensivpatienten venöse Zugänge meistens angelegt sind, wurde nur ein Berechnungsmodus mit der Prozessoptimierung erhoben. Danach wäre realistisch von einer Durchführungszeit von 33:59 Minuten und im optimistischen Fall von 17:57 Minuten auszugehen. 


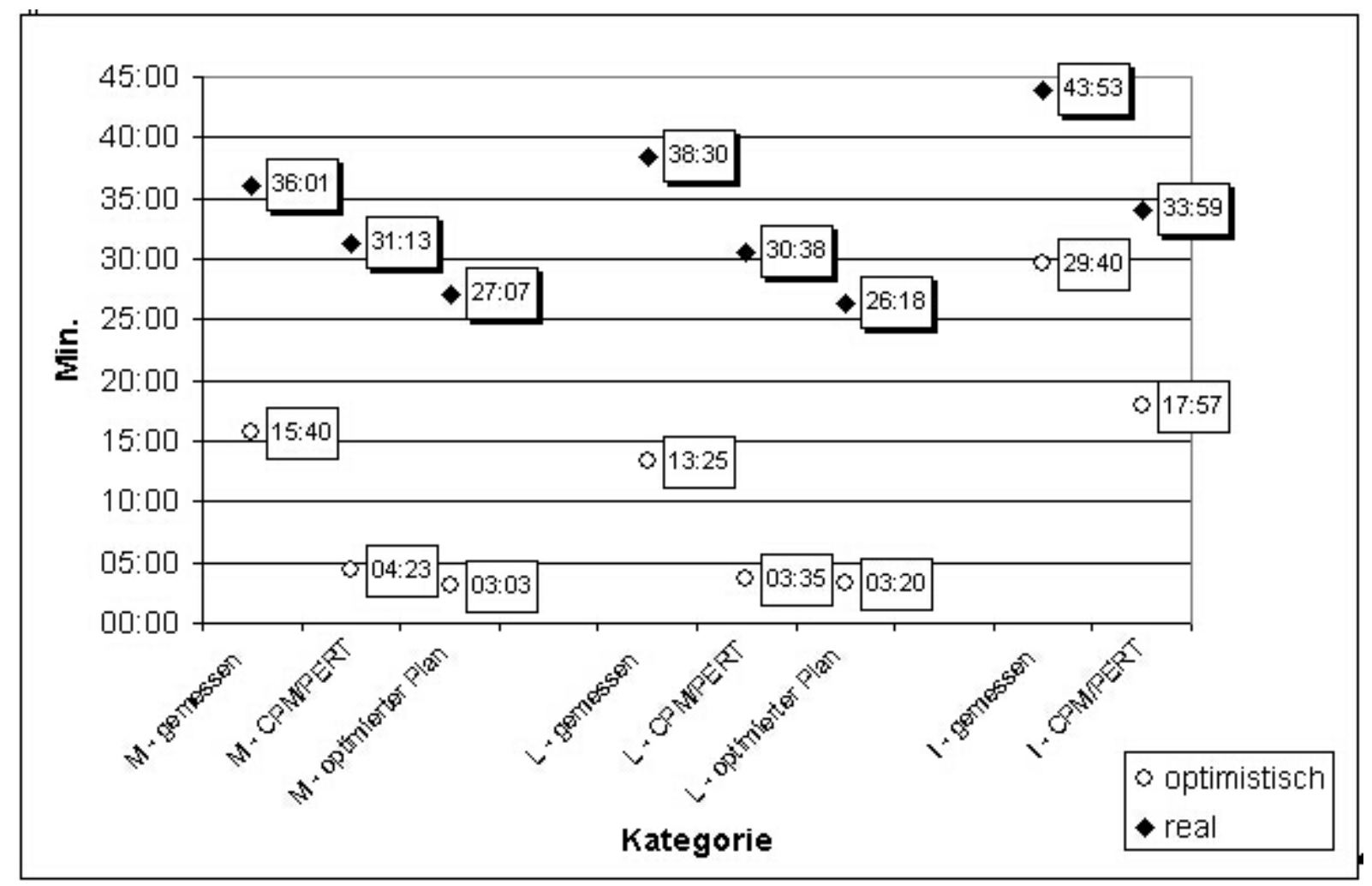

Abbildung 4-20: Vergleich der Durchführungsdauer bei unterschiedlichen Patientenkategorien; $\mathrm{M}$ : mobile Patienten; L: bettlägerige Patienten; I: Intensivpatienten (CT S, CT Thorax).

\subsubsection{Diskussion}

\subsubsection{Zeitmanagement}

Mit dem Prozesszeitmanagement wird der kritische Erfolgsfaktor „Zeit“ gleichrangig mit den Kosten und der Qualität in Zusammenhang gebracht. Die Grundphilosophie des Time-Based Managements (TBM) basiert auf der Erkenntnis, dass eine gesteigerte Kundenzufriedenheit bei gleichzeitig reduzierten Produktkosten und gleichbleibendem Qualitätsniveau nur durch einen effizienteren Umgang mit der Ressource Zeit erreicht werden kann. Ziel ist es, durch Beschleunigung aller Prozesse Wettbewerbsvorteile zu erschließen

Im Mittelpunkt des TBM steht die Optimierung aller Arbeitsabläufe hinsichtlich ihrer Durchlaufzeit. Entscheidend beim Prozesszeitmanagement ist die Optimierung der Ablauforganisation und eine verbesserte Abstimmung von Schnittstellen. Die Existenz eines Krankenhauses hängt zwar nicht unmittelbar von der Patientenfreundlichkeit ab, jedoch besteht aus o. g. Gründen Handlungsbedarf. Reduktion von Wartezeiten, intensivere Betreuung und verbesserte Durchlaufzeiten werden durch höhere Arbeitsproduktivität erreicht, welche aus effektiverer 
Prozessbearbeitung, Eliminierung nicht wertschöpfender Prozesse oder durch bessere Abstimmung der Prozessschnittstellen resultiert. Sie sind ein sind ein entscheidender Schritt in Richtung Patientenfreundlichkeit und gehen tendenziell mit einer Kostenreduktion einher, womit mittel- bis langfristig eine finanzielle Entlastung des Krankenhauses erreicht wird

Der zentrale Punkt des Prozesszeitmanagements besteht in der Planung, Steuerung, Kontrolle und somit Optimierung der Durchlaufzeit. Es lässt sich zwischen Durchlaufzeiten von Prozessen und Prozessketten differenzieren, je nachdem, ob die Abläufe linear oder vernetzt sind, ergibt sich die Durchlaufzeit einer Prozesskette aus der Aggregation der Durchlaufzeiten linear aneinandergereihter Prozesse oder aus der Aggregation der Durchlaufzeiten der Prozesse, die auf dem kritischen Pfad liegen, dem längsten Weg durch das Prozessnetz. Die möglichen Beschleunigungsmaßnahmen setzen entweder an den einzelnen Elementen einer Prozesskette, also an den Prozessen selbst an oder zielen auf eine Optimierung der Ablaufstruktur der Prozessketten.

\subsubsection{Verkürzung der Projektdauer unter Verwendung von CPM}

Die für ein Projekt durchgeführte Zeitplanung wird nicht immer den Vorstellungen der Projektleitung gerecht. Die errechnete Projektdauer kann über dem gewünschten oder verlangten Wert liegen. Man wird dann eine Verkürzung der Projektdauer anstreben.In einer solchen Situation zeigt sich einer der wesentlichen Vorteile der Netzplantechnik. Der kritische Weg weist genau aus, an welchen Stellen Maßnahmen zur Reduzierung der Projektdauer oder der Dauer von Projektteilen ergriffen werden

müssen.

Die Anpassung eines aufgestellten Zeitplans an Terminvorgaben wird immer am kritischen Weg ansetzen und versuchen, die Dauer der auf dem kritischen Weg liegenden Vorgänge zu verkürzen oder die Länge des kritischen Weges durch andere Maßnahmen zu reduzieren. Ohne an dieser Stelle auf Kosten- und Kapazitätsfragen einzugehen, muss allerdings festgestellt werden, dass eine Verkürzung des ursprünglich kritischen Weges immer nur so lange zu einer Verkürzung der Projektdauer führen wird, wie dadurch nicht andere Wege kritisch werden.

Eine Reduzierung der Dauer eines Projektes oder eines Teilprojektes (bei Terminproblemen mit Meilensteinen) kann auf unterschiedliche Art erreicht werden. 


\subsubsection{Verringerung der Ausführungsdauer von kritischen Vorgängen}

Die erste Möglichkeit der Projektdauerverkürzung besteht in der Verringerung der Ausführungsdauer von kritischen Vorgängen. Die Möglichkeiten zur Beschleunigung der Durchführung von Vorgängen hängen mit den Anpassungsformen aus der betriebswirtschaftlichen Produktionswirtschaft zusammen. Hier wird unterschieden zwischen:

1. zeitlicher Anpassung (Abbau von Reserven, Überstunden, Zwei- oder DreiSchichtbetrieb);

2. quantitativer Anpassung (Erhöhung der Kapazitäten);

3. intensitätsmäßiger Anpassung (schneller laufende Maschinen) und

4. qualitativer Anpassung (Übergang zu einem anderen Verfahren).

Die durchgeführten Netzplanberechnungen zur zeitlichen Verringerung des kritischen Weges lassen sich kurz mit der Aussage zusammenfassen: um auf dem kritischen Weg zeitsparend zu sein, sollten sich die beteiligten Arbeitskräfte immer an der aktuellen Untersuchung, und damit am Patienten selber, orientieren. Wie in Abbildung 4-1 dargestellt, sollten kritische Vorgänge während der Durchführungszeit aus dem Untersuchungsraum herausgehalten werden. So können wir mit dem Arbeitsschritt 1e - Zugang legen - deutlich an kritischer Zeit innerhalb des Untersuchungsraumes einsparen, wenn dieser Arbeitsschritt vor dem Eintreten des Patienten in den Untersuchungsraum durchgeführt wird. Eine Reduktion um 24,7\% von 36:01 Minuten (gemessene Dauer) auf 27:07 Minuten in der realistischen Durchführung am Einzeilen-Spiral-CT könnte somit erreicht werden. Optimistisch ist die Reduktion mit 80,5\% von 15:40 Minuten auf 3:03 Minuten noch eindrucksvoller. Damit werden auch weitere Überlegungen relevant: MTRAs sollten dazu ausgebildet werden, selbständig peripher venöse Zugänge legen zu können. Und mit dem Einzug von PACS in die radiologische Diagnostik entfallen des weiteren die kritischen Schritte 3l und 2o während der Nachbereitung. Der Radiologe kann schon während der Untersuchung in einem anderen Raum Befundungen durchführen. 


\subsubsection{Parallele Durchführung der Vorgänge}

Eine wichtige Möglichkeit, die Gesamtdauer eines Projektes oder eines Teilprojektes zu verkürzen, ist die Überlappung von Vorgängen, d.h. die teilweise zeitlich parallele Durchführung der Vorgänge.

\subsubsection{5 Änderung der Ablaufstruktur}

Eine weitere Möglichkeit zur Verkürzung der Projektdauer besteht in einer Änderung der Ablaufstruktur. Die Reihenfolge von Vorgängen ist nicht immer zwingend vorgeschrieben. Dadurch gibt es viele Möglichkeiten, die Ablaufstruktur eines Projektes zu planen und in einem Netzplan wiederzugeben. Durch Änderung der Reihenfolge von Vorgängen und eventuell Parallelschalten von Vorgängen kann fast immer eine Herabsetzung der ursprünglich geplanten Projektdauer erreicht werden. Allgemeingültige Regeln lassen sich hier nicht angeben. Jedoch ist auf einen Tatbestand besonders hinzuweisen. Die Änderung der Reihenfolge von Vorgängen muss häufig auch im Zusammenhang mit Kapazitätsfragen und der Verkürzung von Vorgangszeiten gesehen werden. Die frühere oder spätere Durchführung eines Vorgangs kann nämlich zur Folge haben, dass dann entgegen dem ursprünglichen Plan zu einem bestimmten Zeitpunkt mehr Arbeitskräfte oder Maschinen zur Verfügung stehen. Damit kann die Vorgangsdauer verkürzt werden, und es verringert sich die Projektdauer, wenn es sich um kritische Vorgänge handelt. Ebenso kann durch Umdisposition von Arbeitskräften oder Maschinen eine Verkürzung von kritischen Vorgängen und damit der Projektdauer erreicht werden.

\subsubsection{Pufferzeiten}

Bei der Betrachtung der einzelnen Vorgänge fallen teilweise sehr lange Pufferzeiten auf. Diese werden in der Praxis für weiter anfallende Aufgaben genutzt. Teilweise dienen sie der Abforderung von neuen Patienten, während die Untersuchung durchgeführt wird. Zum anderen nutzt der Radiologe die Zeit zum Nacharbeiten alter Befunde oder für andere Aufgabenfelder. Dabei sollten jegliche anfallende Pufferzeiten zur Reduzierung der Zeit auf dem kritischen Weg durch Unterstützung der Arbeitskraft genutzt werden. Diese Fokussierung auf die wesentlichen Arbeitsschritte hat besonders während der Untersuchung absoluten Vorrang. Förderlich ist weiterhin das Wissen um die eigenen Aufgaben und ihre Bedeutung im 
Arbeitsablauf. Dennoch sollte jede Arbeitskraft die Arbeit der anderen abnehmen bzw. übernehmen können.

\subsubsection{Reduktion der Varianz / Verwendung von PERT}

Zur präziseren Planung der Untersuchungsdauer sollte die Dauer der Vorgänge exakt berechenbar werden. Dazu wurde ein eingehender Blick auf die Varianz der optimistischen und pessimistischen Dauer geworfen. Dabei zeigte sich beim Radiologen eine erhöhte Varianz während der Vorbereitung mit der Aufklärung des Patienten (1b) und dem Legen des Zugangs (1e). Auch das Gespräch mit dem Patienten im Untersuchungsraum (1j) gehört bei sachgemäßer Aufklärung zur Vorbereitung. Um diese Varianz zu reduzieren, sollten diese Arbeitsschritte mit einem zeitlichen Abstand zur unmittelbaren Untersuchung stattfinden. Vorstellbar wäre ein Zeitraum von 30 Minuten. Wie bereits erwähnt, sind dies darüber hinaus Vorgänge, die durchaus von einer Assistentin nach entsprechender Zusatzausbildung durchführbar sind. Zur schnelleren und qualitativ besseren Befundung der Bilder $(1 \mathrm{k}, 1 \mathrm{p})$ sollte ein PACS-gestütztes System installiert werden, das die räumliche Unabhängigkeit und schnellere Bearbeitung fördert. Der Befund kann somit auch zeitlich flexibler durchgeführt werden. Eine vergrößerte Varianz ließe sich bei der ersten Assistentin verringern, wenn eine gezielte Vorbereitung des Untersuchungsraumes (2f) und Platzierung des Patienten auf dem Untersuchungstisch $(2 \mathrm{~g})$ stattfindet. Weiterhin ist zu überlegen, inwiefern der vorhandene Abrechnungsmodus im RIS (30) noch zeitgemäß ist. Eventuell ließe sich dieser Schritt mit den Daten des Computertomographen

koppeln.

Ein weiterer Vorgang, der zur Diskussion steht, ist das Entwickeln, Abholen und Sortieren der Bilder (3l). Dieser für die Varianz sehr anfällige Arbeitsschritt sollte in der nächsten Zeit mit der Durchsetzung der digitalen Radiologie überholt sein. Am problematischsten in Bezug auf die Varianz bleibt dennoch die Untersuchung (3g). Selbst wenn gewisse Definitionen dieses Vorganges von den Zeitnehmern unterschiedlich bewertet wurden, so stellt dieser Arbeitsschritt die anfälligste Komponente des ganzen Prozesses dar. Hier gilt es, potenziell schwierige Untersuchungen vor dem Betreten des Untersuchungsraum zu beurteilen und gezielte Durchführungsprotokolle zu entwerfen, um etwaige pessimistische Zeiten zu minimieren. 
Zusammenfassend lässt sich feststellen, dass eine präzise Vorbereitung die Varianz innerhalb des Untersuchungsraumes in den meisten Fällen senken und glätten wird.

\subsubsection{Durchführungszeiten in unterschiedlichen Patientenkategorien}

Bei dem Vergleich der Patientenkategorien fallen die wenig unterschiedlichen Durchführungszeiten zwischen den mobilen Patienten und den bettlägerigen Patienten auf. Dies betrifft sowohl die realistische wie optimistische Berechung. Wider Erwarten sind die Zeiten für bettlägerige Patienten bis zu 49 Sekunden kürzer. Gründe für die vergleichbaren Ergebnisse in diesen beiden Patientenkategorien liegen zum einen in den zeitlich kaum zu unterscheidenden Vorgängen $2 g$ - Betten des Patienten auf dem Untersuchungstisch - und $2 m$ - Patienten vom Untersuchungstisch bitten, bzw. umlagern. Zum anderen ist der restliche Untersuchungsablauf in beiden Kategorien vergleichbar. Die Kategorie der Intensivpatienten ist bei der Lagerung und Überwachung des Patienten während der Untersuchung mit größerer Varianz behaftet. Dies ist für die Terminplanung eines Computertomographen von entscheidender Bedeutung und legt die Empfehlung nahe, den Bereich - soweit die Möglichkeiten vorhanden sind räumlich und maschinell, und daher organisatorisch von den beiden anderen Kategorien zu trennen. Empfehlenswert ist in diesem Zusammenhang die kurzzeitige Mobilisierung von mehreren Mitarbeitern, damit die Untersuchungszeit nicht nur der Dringlichkeit der Situation entsprechend, sondern auch im Sinne einer optimierten Auslastung klein gehalten wird. Im Bereich der mobilen und bettlägerigen Patienten, die auch als ambulante und stationäre Patienten definiert werden können, ist die Verfügbarkeit der Patienten für eine reibungslose Terminierung von entscheidender Bedeutung. Da dies im Falle von stationären Patienten mit gewissen Einschränkungen verbunden sein kann, wie zum Beispiel durch den Abruf von der Station, durch andere mögliche Untersuchungen oder durch einen langsamen Bettentransport innerhalb des Krankenhauses, ist auch hier eine Trennung der Kategorien und eine eindeutige Zuordnung zu einzelnen Computertomographen als sinnvoll zu erachten. 


\subsubsection{Vorschläge zur Optimierung der Arbeitsabläufe im computertomographischen Arbeitsbereich}

\section{Verbesserung der Rahmenbedingungen}

Optimierung der Anmeldung und Patientenorganisation: am Virchow-Klinikum der Humboldt-Universität zu Berlin stehen vier CT- Geräte zur Verfügung. Bisher erfolgte keine spezielle Aufteilung von Untersuchungen an den Geräten. Seit kurzem ist ein CT-Gerät nur noch für Interventionen wie Punktionen vorgesehen. Zwei CT-Geräte werden ohne besondere Berücksichtigung der Patientenkategorie und des Patientenzustandes betrieben. Um die Auslastung $\mathrm{zu}$ steigern wäre es beispielsweise sinnvoll, nur noch mobile Patienten- sowohl von außerhalb als auch stationäre- am schnellsten CT-Gerät und alle zeitaufwendigeren Patienten an einem nicht so schnellen CT-Gerät zu untersuchen. Darunter fielen Kinder, liegende und intensivpflichtige Patienten. Die Mehrzeilen-CT-Geräte könnten somit vormittags effizient ausgelastet sein und stünden nachmittags ebenfalls für vorig genannte Patientenkategorien und speziellere Interventionen zur Verfügung. Für ein CT-Gerät müssten im Tagesprogramm jeweils zeitliche Freiräume für eventuelle Notfalluntersuchungen vorbehalten sein. Würde man an den beiden benachbarten CT-Geräten je einen Radiologen einsetzen, könnte die Befundung der Untersuchungen beider Geräte in dem Raum zwischen den CTs durchgeführt werden. Wenn ein Radiologe nur für die Befundung und für die Vorbereitung der Untersuchung (Aufklärungsgespräch und intravenöse Zugänge) zuständig wäre, ließe sich das Problem der Wartezeiten bedingt durch den Arzt lösen. Die Aufteilung von Befundung und Aufklärung/ Zugang legen unter den Ärzten müsste selbstverständlich in halb- oder ganztäglichem Wechsel erfolgen. Mit regelmäßig rechtzeitig stattfindender Befundung würde sich die Zahle der Nachfragen der Stationsärzte nach Befunden ebenfalls verringern. Eine Arbeitsüberlastung der bisher zwei eingesetzten MTRAs ließe sich verhindern, indem eine weitere an dem Gerät beschäftigt würde. Die dritte MTRA wäre vor allem für das zeitgerechte Abrufen von Patienten und das an den Untersuchungsplan angepasste Koordinieren von Terminen zuständig. So würde eine weitere Möglichkeit der Organisationsoptimierung eröffnet. Die Änderungen gingen nicht auf Kosten der Mitarbeiter, da ein ausgelastetes Programm mit drei MTRAs die üblichen Erholungspausen sicherstellt. Die Überprüfung der Laborwerte (aktueller Kreatinin und basaler TSH-Wert) ließe sich schon vorher durch das Personal der Anmeldung abfragen und könnte den 
MTRAs gleich über Anmeldung im radiologischen Infromationssystem (RIS) mitgeliefert

werden.

Die Aufklärung über die Kontrastmittelnebenwirkungen und da damit verbundene Legen eines intravenösen Zuganges sollte nicht mehr im Untersuchungsraum geschehen, sondern bereits vorher in einem an den Untersuchungsraum grenzenden Vorbereitungsraum erfolgen. Die Vorteile liegen auf der Hand: die KM- Aufklärung findet nicht mehr auf dem Flur statt, es herrscht eine privatere, für den Patienten angenehmere Atmosphäre und wenn ein Zugang schwierig zu legen ist, verzögert dieser Umstand nicht die Wartezeiten: das CT-Gerät bleibt für andere Patienten frei. 


\section{Effizienzsteigerung durch eine digitale Infrastruktur an einem Routineultraschallarbeitsplatz- Eine quantitative Abschätzung mittels Prozesssimulation}

\section{$5.1 \quad$ Einleitung}

Mit Einführung der DRG sind die medizinischen Leistungserbringer bestrebt, ihre Effizienz zu erhöhen. Wirtschaftliches Arbeiten wird zunehmend zur Existenzfrage. Die Radiologie bringt bedingt durch ihre Aufgabenstellung und ihre Abläufe ein hohes Potential zur

mit.

Der Radiologe erbringt eng definierte Dienstleistungen in der Regel im Auftrag von anderen FachKliniken. Anders als im stationären Versorgungsbereich muss er nur in eingeschränktem Maß auf das schwer vorhersagbare Symptomverhalten des Patienten eingehen. Dies erlaubt genau definierte Abläufe, die sich nach den Erfordernissen der Fragestellung richten. Radiologie ist Informationsverarbeitung, und ein effizientes Arbeiten erfordert ein effizientes Informationsmanagement. Die Idee der Einführung eines PACS (Picture archiving and communication system) zur elektronischen Bildarchivierung liegt nahe. Häufig schrecken jedoch die hohen Investitionskosten ab, zumal der Effizienzgewinn bisher nicht beziffert werden konnte. Auch ist häufig die Erfahrung gemacht worden, dass die Betriebskosten nach Einführung eines PACS stiegen und Arbeitsabläufe komplexer statt einfacher wurden.

Die vorliegende Arbeit adressiert die Frage, wie groß der anvisierte Effizienzgewinn durch eine digitale Infrastruktur tatsächlich ist. Betrachtet wurde ein Routine Ultraschallarbeitsplatz, an dem die Informationsverarbeitung derzeit in konventioneller Weise mit Papierakten und auf Film archivierten Ultraschallbildern abläuft. Um diesen Arbeitsablauf zu analysieren, wurde er als Modell im Computer abgebildet und simuliert. Anschließend wurde das Modell modifiziert im Sinne einer digitalen Infrastruktur und wieder simuliert. Die Ergebnisse der Simulationen wurden miteinander verglichen.

Die hier verwendete Methode der Prozesssimulation ist seit den 40er Jahren des letzten Jahrhunderts entwickelt worden. Im industriellen und Dienstleistungsbereich ist sie seit rund zwei Jahrzehnten etabliert, um Abläufe zu analysieren, verbessern oder in der Planungsphase von vorneherein effizient zu gestalten $(8 ; 17)$. Auch in der Medizin wurde sie bereits verwendet $(16 ; 20 ; 60)$. Sie wird trotz der relativ aufwendigen und damit kostenintensiven Methodik eingesetzt, da sie Zusammenhänge erkennen lässt und Fehler vermeiden hilft, bevor sie gemacht 
werden.

\subsection{Material und Methoden}

Der Arbeitsablauf einer Routineultraschalluntersuchung in einer radiologischen Klinik wurde analysiert und als dynamisches Modell im Computer abgebildet. Anhand dieses Modells wurde der Einfluss einer bisher noch nicht bestehenden digitalen Infrastruktur auf die Effizienz und Personalkosten des Arbeitsablaufs untersucht.

\subsubsection{Beschreibung des untersuchten Ultraschallarbeitsplatzes}

Der beobachtete Arbeitsplatz ist Teil des diagnostischen Funktionsbereichs einer Universitätsklinik. Es stehen drei Sonographiegeräte in drei Untersuchungsräumen zur Verfügung. Der Arbeitsplatz ist als Routine- und Volumenarbeitsplatz konzipiert. Laut Personalschlüssel sind inm ein Oberarzt, ein Assistenzarzt und ein Anfänger, in der Regel ein Arzt im Praktikum, zugeteilt. Bei Beginn der Studie lag der Erfahrungsstand des Oberarztes bei ca. 40.000 Untersuchungen, des Assistenzarztes bei ca. 1200 Untersuchungen, des Anfängers bei ca. 250 Patienten aus dem gesamten Klinikum werden mit verschiedensten Fragestellungen sonographisch untersucht. Dabei kommt die B-Bild-Untersuchung ebenso zum Einsatz wie die farbkodierte Duplexsonographie. Zwischen dem 1.3.2000 und dem 28.2.2001 wurden pro normalem Arbeitstag im Routinebetrieb (Wochentage ohne Feiertage, Ankunft der Patienten zwischen 8:00 und 16:00 Uhr) durchschnittlich 37,6 [9-57] Untersuchungen an durchschnittlich 30,7 [9-48] Patienten durchgeführt. Häufigste Untersuchung war mit 8,1 pro Tag die Sonographie des Abdomens. Ankommende Patienten werden von den medizinisch-technischen Röntgenassistenten (MTRA) registriert und für die Untersuchung vorbereitet. Ärzte führen die Ultraschalluntersuchung durch und erstellen die Untersuchungsbefunde computergestützt in vordefinierten Bildschirmformularen. Die ausgedruckten Befunde werden von den MTRAs zusammen mit den Ultraschallbildern archiviert und in Kopie den Patienten ausgehändigt. Die Administration mit Zeitplanung und Abrechnung erfolgt computergestützt über das klinikinterne radiologische Informations- und Abrechnungssystem (RIS). Befunde und Bilder werden konventionell archiviert. 


\subsubsection{Digitale Infrastruktur}

Im Unterschied zum vorhandenen Arbeitsplatz ist bei der anvisierten digitalen Infrastruktur ein größtmögliches $\mathrm{Ma}$ an Information digital verarbeitet und automatisiert. Unser Vorgabe für das Simulationsmodell umfasst ein digitales Bildarchiv (PACS), eine digitale Patientenakte und eine automatisierte Terminverwaltung. Bisher ist kein PACS und Terminverwaltung auf Basis eines elektronischen Terminkalenders ohne Automatismen verfügbar.

\subsubsection{Prozesssimulation}

Mit der Prozesssimulation wird die Dynamik des Arbeitsablaufs eines sonographischen Routinearbeitsplatzes visualisiert und evaluiert. Grundlage für die Simulation ist ein Prozessmodell. Dieses besteht aus der Prozessstruktur, Ressourcen und Flussobjekten:

1. Die Prozessstruktur setzt sich aus Vorgängen (Arbeitsschritten) mit ihren Abhängigkeiten zusammen (Wann findet welcher Vorgang statt?). Vorgange sind charakterisiert durch inr Dauer.

2. Ressourcen sind die Arbeitskraft und/oder das Material, die für die Durchführung einer Aktivität nötig sind. Ihre Zuteilung erfolgt in den Einheiten [Arbeitskraft x Zeit] bzw. [Material x Zeit].

3. Die Flussobjekte bewegen sich entlang der Prozessstruktur. Flussobjekte entsprechen z.B. Patienten und Akten, die bearbeitet werden. Ihr Verhalten in der Prozessstruktur wird von den Eigenschaften der Vorgänge, deren Beziehungen untereinander, der Ressourcenverfügbarkeit sowie von ihren eigenen Eigenschaften bestimmt und ermöglicht Aussagen über die Prozessqualität. 
Tabelle 5-1: Erstellung eines Prozessmodells in fünf Phasen

\begin{tabular}{|l|l|}
\hline Phase & Tätigkeiten \\
\hline I Zielsetzung \\
definieren & $\begin{array}{l}\text { Problem formulieren } \\
\text { Fragestellung ableiten } \\
\text { Genaue Ziele definieren }\end{array}$ \\
\hline II Strukturermittlung & $\begin{array}{l}\text { Vorgänge ermitteln (Was wird gemacht?) } \\
\text { Ressourcenallokation (Wer macht was?) } \\
\text { Logische Beziehungen zwischen den Vorgängen } \\
\text { ermitteln (Wann wird etwas gemacht?) } \\
\text { Flussobjekte mit ihren Eigenschaften ermitteln (Mit wem } \\
\text { wird etwas gemacht?) }\end{array}$ \\
\hline III Messdatenerfassung & $\begin{array}{l}\text { Zeitmessung der einzelnen Vorgänge } \\
\text { Auftretenshäufigkeit der einzelnen Vorgänge ermitteln } \\
\text { Aufbereitung der Messwerte }\end{array}$ \\
\hline IV $\quad \begin{array}{l}\text { Prozessmodell } \\
\text { anfertigen }\end{array}$ & $\begin{array}{l}\text { Abbildung der Struktur und Messergebnisse in einem } \\
\text { Prozessmodell } \\
\text { Verifizierung, Validierung }\end{array}$ \\
\hline V Auswertung & $\begin{array}{l}\text { Verschiedene Szenarien implementieren } \\
\text { Untersuchungsdauer } \\
\text { Beliebige Teilprozessdauern }\end{array}$ \\
\hline
\end{tabular}

\subsubsection{Phase I}

Die Zielsetzung entscheidet über Umfang und Detailgrad des Simulationsmodells. Für ein Simulationsprojekt wird das anfangs oft subjektiv wahrgenommene Problem genau formuliert. Die Problemstellung ermöglicht eine exakte Fragestellung, die wiederum als Grundlage für die Zielsetzung des Projekts dient. Mit dieser Studie wurde wahrgenommene Ineffizienz durch die papierbasierte Aktenführung und konventionelle Bildarchivierung bei hohen Patientenaufkommen 
adressiert. Sie mündete in der Frage, wie viel Arbeitsaufwand durch die Einführung einer digitalen Infrastruktur eingespart werden kann. Dazu sollte ein Modell erstellt werden, welches eine zutreffende Aufwands und Kostenanalyse des bestehenden und des prospektierten Arbeitsablaufs ermöglicht. Das Prozessmodell sollte den Vergleich der Kenngrößen bei Szenarien mit und ohne digitale Infrastruktur ermöglichen. 
Tabelle 5-2: Indikatoren der Prozessleistung

\begin{tabular}{|c|c|c|}
\hline Indikator & Einheit & Beschreibung \\
\hline \multicolumn{3}{|l|}{ Taktzeiten (T) } \\
\hline Ultraschallgeräte $\quad T_{G}$ & $\begin{array}{l}\text { [min:se } \\
\text { c] }\end{array}$ & $\mathrm{T}_{\mathrm{G}}=\frac{\text { Zeit für alle Untersuchungen }}{\text { Anzahl der Untersuchungen }} \cdot$ Anzahl Geräte \\
\hline \multicolumn{3}{|c|}{ Patientendurchlaufzeiten (P) } \\
\hline Anwesenheit & $\begin{array}{l}\text { [min:se } \\
\text { c] }\end{array}$ & $\begin{array}{l}\text { Ankunft des Patienten bis Aushändigung des } \\
\text { Befunds }\end{array}$ \\
\hline Untersuchungszeit $\mathrm{P}_{U}$ & $\begin{array}{l}\text { [min:se } \\
\text { c] }\end{array}$ & Dauer der Untersuchung am Patienten \\
\hline $\begin{array}{l}\text { Wartezeit vor } \\
\text { Untersuchung }\end{array}$ & $\begin{array}{l}\text { [min:se } \\
\text { c] }\end{array}$ & $\begin{array}{l}\text { Ankunft des Patienten bis Beginn der } \\
\text { Untersuchung }\end{array}$ \\
\hline $\begin{array}{ll}\text { Wartezeit nach } & \mathrm{P}_{\mathrm{W}} \\
\text { Untersuchung } & \mathrm{n}\end{array}$ & $\begin{array}{l}\text { [min:se } \\
\text { c] }\end{array}$ & $\begin{array}{l}\text { Zeit von Ende der Untersuchung bis } \\
\text { Aushändigung des Befunds }\end{array}$ \\
\hline \multicolumn{3}{|l|}{ Arbeitsaufwand (A) } \\
\hline $\begin{array}{l}\text { Gesamtaufwand pro } \mathrm{A}_{\mathrm{G}} \\
\text { Untersuchung }\end{array}$ & $\begin{array}{l}\text { [min:se } \\
\text { c] }\end{array}$ & $A_{G}=\frac{\text { Summe aller Aktivitätsdauern }}{\text { Anzahl der Untersuchungen }}$ \\
\hline \multicolumn{3}{|c|}{ Ressourcenauslastung (R) } \\
\hline Arbeitskräfte & {$[\%]$} & $\mathrm{R}_{\mathrm{A}}=\frac{\text { aktiveZeitderArbeitskraft }}{\text { Anwesenheitszeit }} \cdot 100$ \\
\hline Ultraschallgeräte & {$[\%]$} & $\mathrm{R}_{\mathrm{G}}=\frac{\text { Untersuchungsdauer }}{\text { Zeit für alle Untersuchungen }} \cdot 100$ \\
\hline
\end{tabular}




\subsubsection{Phase II: Strukturermittlung}

Für die Strukturermittlung begleitete ein nicht in den Arbeitsablauf eingebundener Beobachter (Arzt) die Untersuchungen von 30 Patienten und notierte alle identifizierten Einzelaktivitäten in einer Vorgangsliste. Wo im Rahmen der Fragestellung vertretbar, wurden mehrere einzelne Vorgänge zu einem übergeordneten Vorgang zusammengefasst, um das Prozessmodell so einfach wie möglich zu halten. Anschließend wurde für jeden Vorgang ermittelt, welche Vorgänge unmittelbar vor ihrer Durchführung ausgeführt sein müssen und welche nachfolgend durchzuführen sind. Eine Aufleistung der einzelnen ermittelten Vorgänge (Aktivitäten) befindet sich in 4-3.

Bei der Protokollierung der Vorgänge wurden die Ressourcen miterfasst. Die ermittelten Daten wurden wiederholt mit den Ärzten und MTRAs abgeglichen, um Zusammenhänge möglichst realitätsnah zu erfassen und Beobachterfehler zu vermeiden.

Tabelle 5-3: Ermittelte Vorgänge einer Ultraschalluntersuchung mit Beschreibung, Ressourcen, Vorgängern, Auftretenshäufigkeit $\left(\mathrm{H}_{\mathrm{A}}\right)$, Anzahl der Messwerte $(\mathrm{n})$, Mittelwert der Messwerte $(\mu \mathrm{MW})$, Mittelwert der simulierten Werte ( $\mu$ Sim.) und Signifikanzniveau (p).

\begin{tabular}{|c|c|c|c|c|c|c|c|c|}
\hline Nr. & Aktivität & $\begin{array}{l}\text { Ressourcen } \\
{[\text { Arb.kraft (Mat.)] }}\end{array}$ & $\begin{array}{l}\text { Vor } \\
\text { g. }\end{array}$ & $\mathrm{H}_{\mathrm{A}}$ & $n$ & MW & $\begin{array}{l}\mu \\
\text { Sim. }\end{array}$ & $p$ \\
\hline 1 & \multicolumn{8}{|l|}{ Untersuchung } \\
\hline $1 a$ & Patient abrufen & MTRA (Telefon) & & $\begin{array}{l}0.6 \\
8\end{array}$ & 18 & $1: 36$ & $1: 38$ & $\begin{array}{l}0,40 \\
5\end{array}$ \\
\hline $1 b$ & $\begin{array}{l}\text { Telef. Rückfr. wg. fehlender } \\
\text { Unterlagen }\end{array}$ & MTRA (Telefon) & $1^{\circ}$ & $\begin{array}{l}0.1 \\
7\end{array}$ & 11 & $1: 50$ & 2:01 & $\begin{array}{l}0,47 \\
2\end{array}$ \\
\hline $1 \mathrm{c}$ & $\begin{array}{l}\text { Patient registrieren; Akte } \\
\text { vorbereiten }\end{array}$ & $\begin{array}{l}\text { MTRA } \\
\text { (Terminal) }\end{array}$ & $\begin{array}{l}1^{\circ} \\
1 \mathrm{~b}\end{array}$ & $\begin{array}{l}1.0 \\
0\end{array}$ & 17 & $1: 59$ & 2:02 & $\begin{array}{l}0,46 \\
1\end{array}$ \\
\hline $1 d$ & Patient suchen & MTRA & $1 c$ & $\begin{array}{l}0.1 \\
6\end{array}$ & 8 & $2: 50$ & $2: 47$ & $\begin{array}{l}0,42 \\
0\end{array}$ \\
\hline $1 e$ & Untersuchung vorbereiten & MTRA & $\begin{array}{l}1 c ; \\
1 d\end{array}$ & $\begin{array}{l}1.0 \\
0\end{array}$ & 38 & 3:07 & $3: 17$ & $\begin{array}{l}0,52 \\
9\end{array}$ \\
\hline
\end{tabular}




\begin{tabular}{|c|c|c|c|c|c|c|c|c|}
\hline Nr. & Aktivität & $\begin{array}{l}\text { Ressourcen } \\
\text { [Arb.kraft (Mat.)] }\end{array}$ & $\begin{array}{l}\text { Vor } \\
\text { g. }\end{array}$ & $\mathrm{H}_{\mathrm{A}}$ & $\mathrm{n}$ & $\begin{array}{l}\mu \\
M W\end{array}$ & $\begin{array}{l}\mu \\
\text { Sim. }\end{array}$ & $\mathrm{p}$ \\
\hline $1 f$ & Anforderung lesen & Arzt & $1 \mathrm{e}$ & $\begin{array}{l}1.0 \\
0\end{array}$ & 16 & $0: 45$ & $0: 44$ & $\begin{array}{l}0,06 \\
9\end{array}$ \\
\hline $1 \mathrm{~g}$ & $\begin{array}{l}\text { Anforderung telefonisch } \\
\text { abklären }\end{array}$ & Arzt (Telefon) & $1 f$ & $\begin{array}{l}0.0 \\
8\end{array}$ & 5 & 5:49 & 7:02 & $\begin{array}{l}0,28 \\
9\end{array}$ \\
\hline $1 \mathrm{~h}$ & Patient untersuchen & $\begin{array}{l}\text { Arzt } \\
\text { (Untersuchungs } \\
\text { raum) }\end{array}$ & $1 \mathrm{f}$ & $\begin{array}{l}1.2 \\
4\end{array}$ & 47 & $\begin{array}{l}16: 0 \\
1\end{array}$ & $\begin{array}{l}15: 2 \\
7\end{array}$ & $\begin{array}{l}0,65 \\
6\end{array}$ \\
\hline $1 \mathrm{i}$ & $\begin{array}{l}\text { Nachuntersuchung durch } \\
\text { Experte }\end{array}$ & $\begin{array}{l}\text { Arzt; Experte } \\
\text { (Untersuchungs } \\
\text { raum) }\end{array}$ & $1 \mathrm{~h}$ & $\begin{array}{l}0.2 \\
2\end{array}$ & 34 & 6:11 & 6:39 & $\begin{array}{l}0,51 \\
5\end{array}$ \\
\hline $1 \mathrm{j}$ & Befund verfassen und drucken & Arzt (Computer) & $\begin{array}{l}1 \mathrm{~h} ; \\
1 \mathrm{i}\end{array}$ & $\begin{array}{l}1.2 \\
4\end{array}$ & 61 & 8:59 & 9:08 & $\begin{array}{l}0,76 \\
7\end{array}$ \\
\hline $1 \mathrm{k}$ & Befund telefonisch übermitteln & Arzt (Telefon) & $1 \mathrm{j}$ & $\begin{array}{l}0.0 \\
3\end{array}$ & 7 & 2:24 & $2: 48$ & $\begin{array}{l}0,16 \\
2\end{array}$ \\
\hline 11 & Untersuchung nachbereiten & MTRA & $1 \mathrm{k}$ & $\begin{array}{l}1.0 \\
0\end{array}$ & 90 & 2:45 & $2: 38$ & $\begin{array}{l}0,17 \\
1\end{array}$ \\
\hline $1 \mathrm{~m}$ & $\begin{array}{l}\text { Befund aushändigen; Patient } \\
\text { versenden }\end{array}$ & MTRA & 11 & $\begin{array}{l}1.0 \\
0\end{array}$ & 24 & $0: 52$ & $0: 49$ & $\begin{array}{l}0,22 \\
5\end{array}$ \\
\hline 2 & Terminverwaltung & & & & & & & \\
\hline $2 a$ & Anforderung beurteilen & Arzt & & $\begin{array}{l}0.8 \\
1\end{array}$ & 6 & 1:03 & 1:07 & $\begin{array}{l}0,01 \\
3\end{array}$ \\
\hline $2 \mathrm{~b}$ & $\begin{array}{l}\text { Anforderung telefonisch } \\
\text { abklären }\end{array}$ & Arzt (Telefon) & $2^{\circ}$ & $\begin{array}{l}0.1 \\
8\end{array}$ & 5 & 5:49 & $7: 26$ & $\begin{array}{l}0,12 \\
8\end{array}$ \\
\hline $2 \mathrm{c}$ & Termin heraussuchen & $\begin{array}{l}\text { MTRA } \\
\text { (Terminal) }\end{array}$ & $2^{\circ}$ & 1.0 & 6 & $0: 59$ & $0: 59$ & $\begin{array}{l}<0,0 \\
1\end{array}$ \\
\hline $2 d$ & Telefonisch Termin klären & MTRA (Telefon) & $\begin{array}{l}2^{\circ} \\
2 b\end{array}$ & $\begin{array}{l}0.8 \\
1\end{array}$ & 23 & 1:36 & 1:40 & $\begin{array}{l}0,96 \\
1\end{array}$ \\
\hline $2 \mathrm{e}$ & Patient anmelden & $\begin{array}{l}\text { MTRA } \\
\text { (Terminal) }\end{array}$ & 2c & $\begin{array}{l}1.0 \\
7\end{array}$ & 57 & $2: 53$ & $2: 58$ & $\begin{array}{l}0,24 \\
2\end{array}$ \\
\hline $2 f$ & Termin verschieben & $\begin{array}{l}\text { MTRA } \\
\text { (Terminal) }\end{array}$ & & $\begin{array}{l}0.0 \\
7\end{array}$ & 6 & 2:48 & 3:37 & $\begin{array}{l}0,08 \\
6\end{array}$ \\
\hline
\end{tabular}




\begin{tabular}{|c|c|c|c|c|c|c|c|c|}
\hline $\mathrm{Nr}$. & Aktivität & $\begin{array}{l}\text { Ressourcen } \\
\text { [Arb.kraft (Mat.)] }\end{array}$ & $\begin{array}{l}\text { Vor } \\
\text { g. }\end{array}$ & $\mathrm{H}_{\mathrm{A}}$ & $n$ & $\mu$ & $\begin{array}{l}\mu \\
\operatorname{Sim} .\end{array}$ & $p$ \\
\hline $2 g$ & Patient abmelden & $\begin{array}{l}\text { MTRA } \\
\text { (Terminal) }\end{array}$ & & $\begin{array}{l}0.0 \\
9\end{array}$ & 5 & 2:00 & 2:07 & $\begin{array}{l}0,31 \\
8\end{array}$ \\
\hline 3 & \multicolumn{8}{|l|}{ Aktenverwaltung } \\
\hline $3 a$ & Akten vorbereiten & MTRA & & $\begin{array}{l}1.0 \\
0\end{array}$ & 60 & 2:44 & 2:59 & $\begin{array}{l}0,55 \\
6\end{array}$ \\
\hline $3 b$ & Akten sonstige Tätigkeiten & MTRA & & $\begin{array}{l}0.2 \\
2\end{array}$ & 18 & $3: 41$ & 3:37 & $\begin{array}{l}0,86 \\
7\end{array}$ \\
\hline $3 c$ & $\begin{array}{l}\text { Telef. Rückfr. wg. fehlender } \\
\text { Unterlagen }\end{array}$ & MTRA (Telefon) & & $\begin{array}{l}0.1 \\
6\end{array}$ & 11 & 1:50 & 2:04 & $\begin{array}{l}0,78 \\
4\end{array}$ \\
\hline 4 & \multicolumn{8}{|l|}{ Filmverwaltung } \\
\hline $4 a$ & Archivtüten bekleben & MTRA & & $\begin{array}{l}1.0 \\
0\end{array}$ & 65 & $0: 41$ & $0: 21$ & $\begin{array}{l}<0,0 \\
1\end{array}$ \\
\hline $4 b$ & Filme vom Drucker holen & MTRA & & $\begin{array}{l}0.1 \\
0\end{array}$ & 2 & $2: 20$ & $2: 20$ & $\mathrm{~N} / \mathrm{a}$ \\
\hline $4 c$ & Filme in Akte sortieren & MTRA & $\begin{array}{l}4^{\circ} ; \\
4 \mathrm{~b}\end{array}$ & $\begin{array}{l}1.0 \\
0\end{array}$ & 47 & 2:08 & $1: 52$ & $\begin{array}{l}<0,0 \\
1\end{array}$ \\
\hline 5 & \multicolumn{8}{|l|}{ Sonstige Tätigkeiten } \\
\hline $5 a$ & Materialnachschub & MTRA & & $\begin{array}{l}0.0 \\
6\end{array}$ & 6 & $3: 47$ & $4: 41$ & $\begin{array}{l}0,02 \\
1\end{array}$ \\
\hline $5 b$ & $\begin{array}{l}\text { Sonstige telef. Anfragen } \\
\text { beantworten }\end{array}$ & Arzt (Telefon) & & $\begin{array}{l}0.2 \\
0\end{array}$ & 11 & 1:57 & 1:54 & $\begin{array}{l}0,91 \\
6\end{array}$ \\
\hline $5 c$ & $\begin{array}{l}\text { Sonstige telef. Anfragen } \\
\text { beantworten }\end{array}$ & MTRA (Telefon) & & $\begin{array}{l}0.3 \\
3\end{array}$ & 33 & 1:18 & $1: 23$ & $\begin{array}{l}0,77 \\
3\end{array}$ \\
\hline
\end{tabular}




\subsubsection{Phase III: Messdatenerfassung}

Mit einem proprietären Softwaretool wurden für jeden Vorgang zwischen 2 und 159 Dauern gemessen. Die Software ermöglichte die Beobachtung mehrerer parallel ablaufender Vorgänge und verkürzte damit die Periode der Messdatenerfassung erheblich

Zusätzlich zu den Zeitmessungen wurde ermittelt, wie häufig Vorgänge pro Zeiteinheit auftraten, die nicht direkt an eine Ultraschalluntersuchung gebunden waren (z.B. ankommende Telefonate, Anmeldevorgänge). Dieser Wert wurde über die Anzahl der Untersuchungen im Beobachtungszeitraum in die durchschnittliche Auftretenshäufigkeit pro Untersuchung umgerechnet. Daten zur Anzahl der Patienten und An- bzw. Abmeldevorgänge pro Tag sowie Art und Anzahl der Untersuchungen pro Tag wurden den Daten eines Jahres aus dem klinikinternen Abrechnungssystem entnommen.

\subsubsection{Phase IV: Prozessmodell anfertigen}

Für die Modellierung und anschließende Simulation verwendeten wir die Software Process v3 der Firma sciforma co. (Los Gatos, CA, USA) (4). Mit den ermittelten Vorgängen und Anordnungsbeziehungen wurde ein Netzplandiagramm erstellt. Pfeile (Aktivitätspfade) repräsentieren den Weg, den ein Flussobjekt im Prozess durchlaufen kann (Abbildung 4-1).

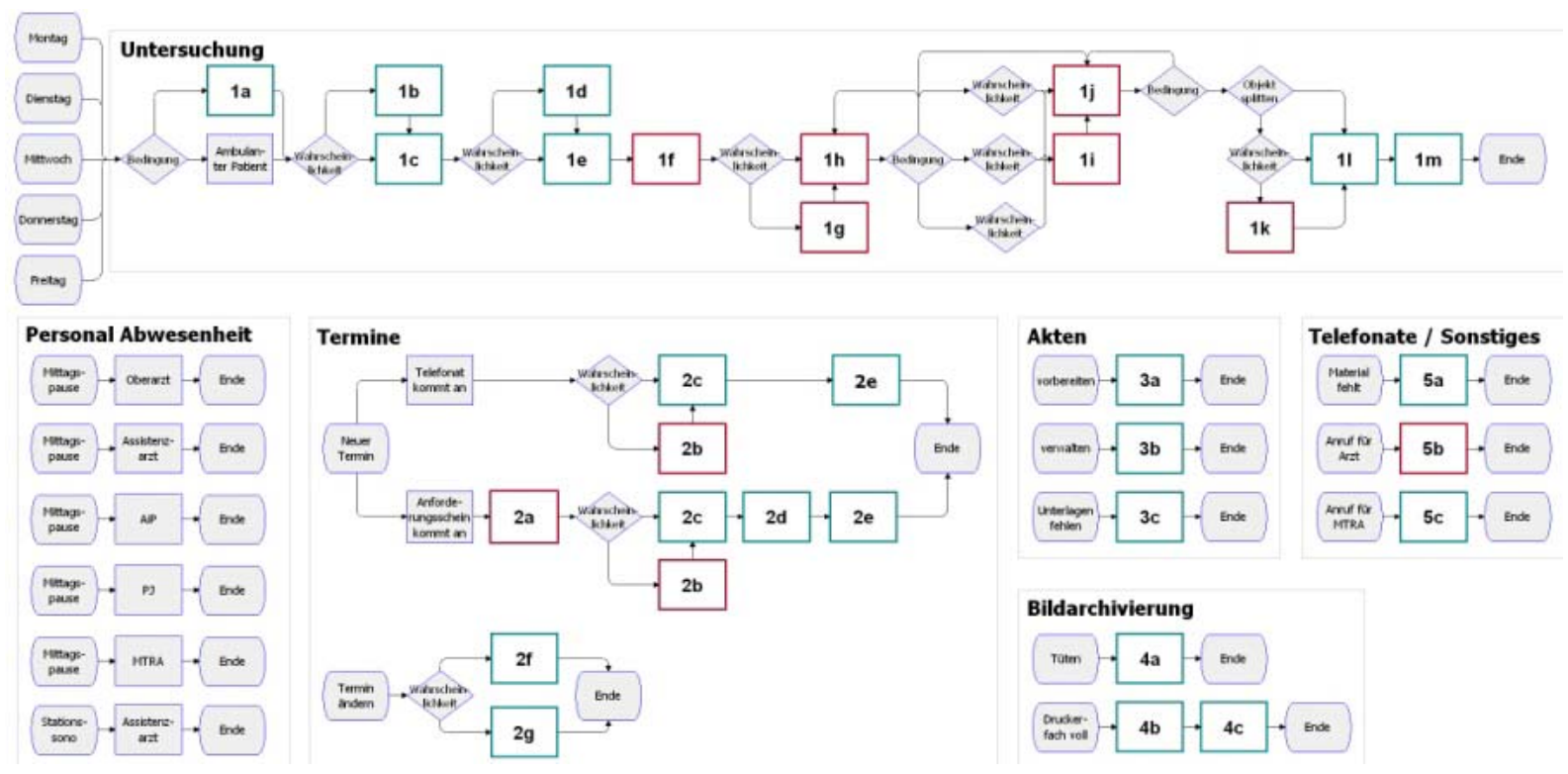


Abbildung 5-1: Flussdiagramm der Prozessstruktur; Evaluationsmodell. Die grau unterlegten Vorgänge dienen der Prozesssteuerung. Prozessstruktur: Vom Evaluationsprozess wurden alle ausbildungsorientierten Vorgänge („1i“ und Steuervorgänge, s. Abbildung 4-1 bis 4-3) entfernt.

Ressourcenallokation: Es wurden drei Experten und zwei erfahrene Untersucher zugewiesen. ortgeschrittene und Anfänger wurden nicht alloziert. Alle anderen

Ressourcen

blieben

gleich.

In der hier gewählten Darstellungsform finden zwei Arten von Aktivitäten Verwendung. Weiße Rechtecke bilden Aktivitäten $a b$, in denen Untersuchungsvorgänge stattfinden. Grau unterlegte Vorgänge repräsentieren Verzweigungspunkte, die den Weg der Flussobjekte steuern. Ihnen sind keine Ressourcen oder Vorgangsdauern zugeordnet. Das Verhalten eines Objekts an Verzweigungspunkten in der Prozessstruktur wurde stochastisch oder mit komplexen Bedingungen modelliert. Den logischen Zusammenhängen der einzelnen Aktivitäten entsprechend entstanden 11 Teilprozesse (z.B. Untersuchung, Termin vereinbaren, Patientenakte vorbereiten etc.). Die eigentliche Ultraschalluntersuchung wird als Hauptprozess bezeichnet. Die eigentliche Ultraschalluntersuchung wird als Hauptprozess bezeichnet. Die Abhängigkeit zwischen den Teilprozessen besteht über die Ressourcenverfügbarkeit sowie über den Auftretenswahrscheinlichkeit. Beispielsweise wurde der Teilprozess ankommende Telefonate entsprechend der gemessenen Häufigkeit im Abstand von etwa 20 min getriggert. Jedem Vorgang wurde die erforderlichen Ressourcen zugewiesen. An Sachmitteln wurden nur solche mit begrenzter Menge und Verfügbarkeit (z.B. Ultraschallgeräte, Befundungscomputer) ins Prozessmodell aufgenommen.

Auf Basis der im Vorfeld erhobenen Daten wurden die Vorgangsdauern stochastisch modelliert. Eine statistische Analysesoftware (5) ermittelte für jede Vorgangsdauer, für die zehn oder mehr Messwerte vorlagen, eine Verteilungsfunktion. Dabei kamen Weibull-, Lognormal-, Erlang- und Betaverteilungen zum Einsatz. In den anderen Fällen wurde eine Dreiecksverteilungsfunktion verwendet, deren Eckpunkte über das Minimum, den Median und das Maximum der gemessenen Vorgangsdauern definiert waren.

Die Anzahl der Flussobjekte (z.B. Anzahl der Patienten) des Hauptprozesses richtet sich nach der Anzahl der zu simulierenden Untersuchungen. Die Anzahl der 
Flussobjekte in den Teilprozessen war in Abhängigkeit zum Hauptprozess modelliert. Im Verlauf der Modellierung wurde die Prozessstruktur wiederholt mit den beteiligten Ärzten und MTRAs verifiziert und angepasst, bis sie dem beobachteten Arbeitsablauf entsprach. Die Übereinstimmung der von der Simulation generierten mit den gemessenen Daten wurde auf zwei Ebenen statistisch validiert $(35,78)$. Die simulierten Vorgangsdauern wurden mittels Goodness-of-Fit-Test (KolmogorovSmirnov) bei einem Signifikanzniveau von $p=0,05$ mit den gemessenen verglichen (Tabelle 4-3). Der Unterschied durfte nicht signifikant sein. Grundlage der zweiten Validierungsebene waren Patientendurchlaufzeiten, die über vier Wochen vom Abrechnungssystem der Klinik automatisch protokolliert worden waren. Sie mussten beim Mann-Whitney-Test beim Signifikanzniveau von $p=0,05$ mit den entsprechenden simulierten Zeitspannen übereinstimmen. Das Modell musste neben der durchschnittlichen Intervalldauer auch die Veränderung der Intervalldauer über den Tag reproduzieren (Abbildung 5-2).

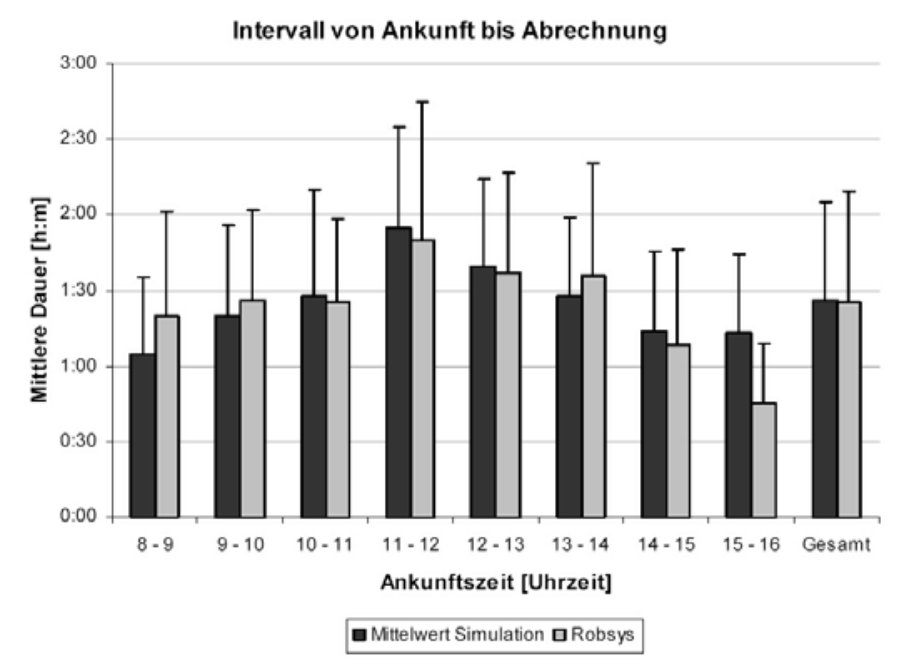

Abbildung 5-2: Validierung der Prozessdaten, Vergleich der gemessenen Patientenlaufzeiten von Patientenregistrierung (Aktivität 1c) bis Nachbereitung der Untersuchung (Aktivität 11) mit den simulieren Zeiten in Abhängigkeit von Tageszeit

\subsubsection{Phase V: Auswertung}

Jeder Prozessdurchlauf simulierte einen Zeitraum von einem halben Jahr (26 Wochen à 5 Arbeitstage). Für die Simulation digitalen Infrastruktur wurde das validierte Prozessmodell abgewandelt. Die Veränderungen betrafen die Prozessstruktur, Vorgangsdauern und Ressourcenallokation. Es wurden somit alle Vorgänge entfernt, die durch die digitale Infrastruktur automatisiert wurden 
(Abbildung 5-3). Es entfielen alle Vorgänge, sie sich auf Akten-, Film- und Terminverwaltung beziehen.

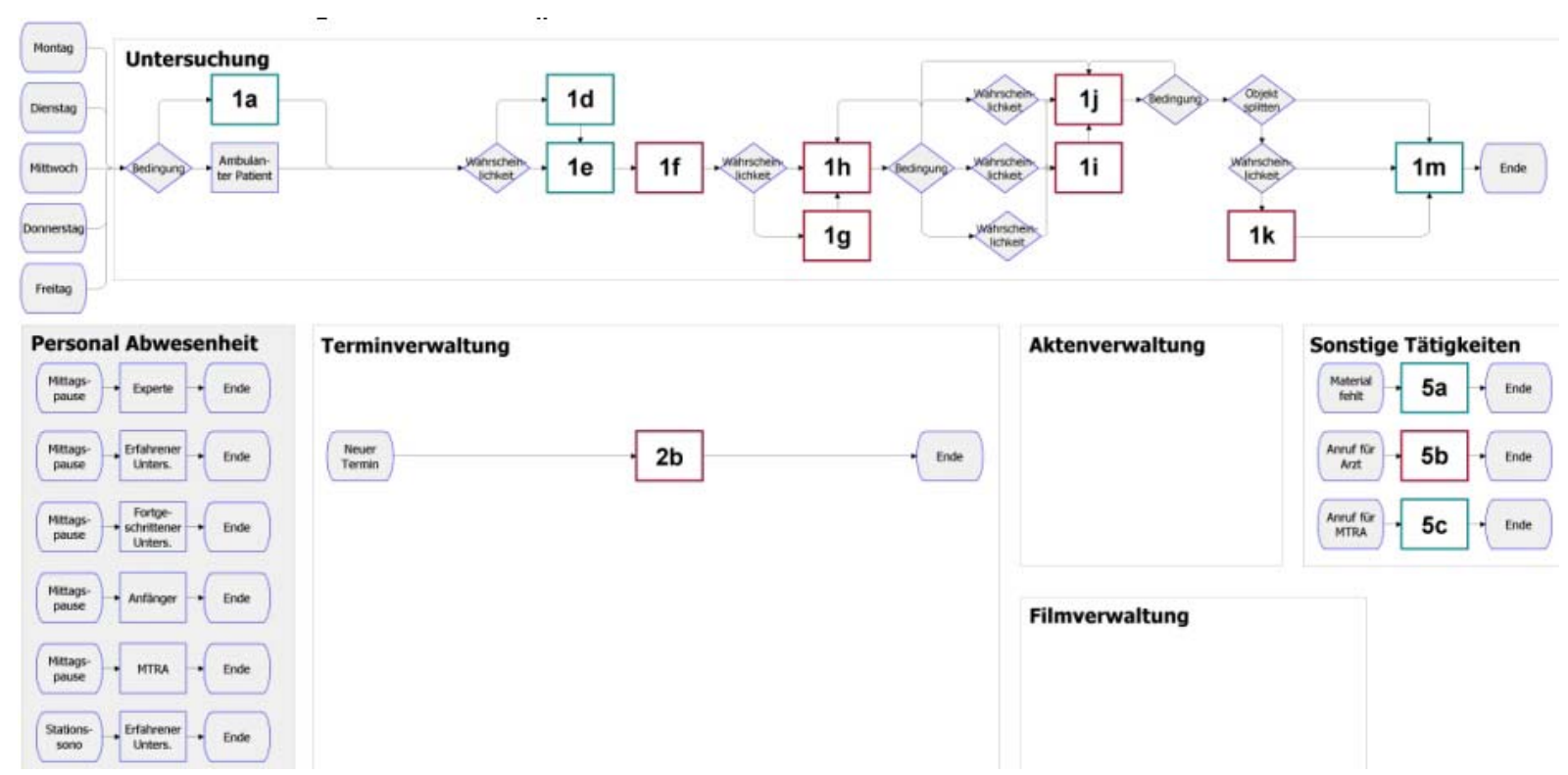

Abbildung 5-3: Prozessstruktur mit digitaler Infrastruktur

Die digitale Infrastruktur wurde mit unterschiedlichen Personalbesetzungen von Ärzten und MTRAs simuliert. Ziel war, eine ausgewogene Kombination aus möglichst hoher Ressourcenauslastung $\left(R_{A}\right)$ bei möglichst kurzer Patientenlaufzeit $\left(P_{A}\right)$ und möglichst geringen Kosten zu erreichen. Den Kostenberechnung lagen für die jeweiligen Personalressourcen die Vergütung nach BAT zuzüglich ortsüblichen Zulagen (Kosten pro Stunde: Assistenzarzt BAT Ila $=19,41 € /$ Facharzt BAT Ib $=$ $21,20 €$ / MTRA BAT Vc $=12,53 €)$ zu Grunde. Materialkosten wurden nicht berücksichtigt.

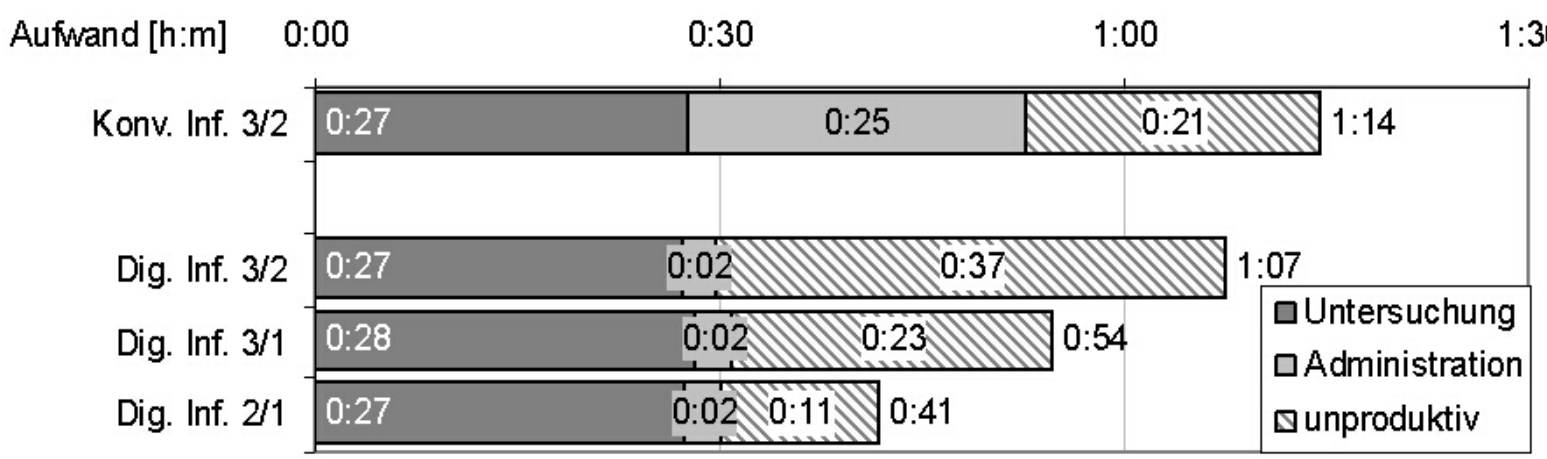


Abbildung 5-4: Arbeitsaufwand für Untersuchungs- und Administrationstätigkeiten sowie unproduktive Zeiten pro Untersuchung. Gegenüberstellung eines Szenariums mit konventioneller Infrastruktur und mehrerer Szenarien mit digitaler Infrastruktur. Unterschiedliche personelle Besetzung (Ärzte/MTRAs).

Bei Einsatz einer digitalen Infrastruktur wird eine ähnliche Gerätetaktzeit und -auslastung bei verringertem Ressourceneinsatz erzielt. Bei gleichem Ressourceneinsatz verringert sich die Taktzeit; die Geräteauslastung und die Anzahl der Patienten pro Zeiteinheit steigt (Abbildung 6-5).

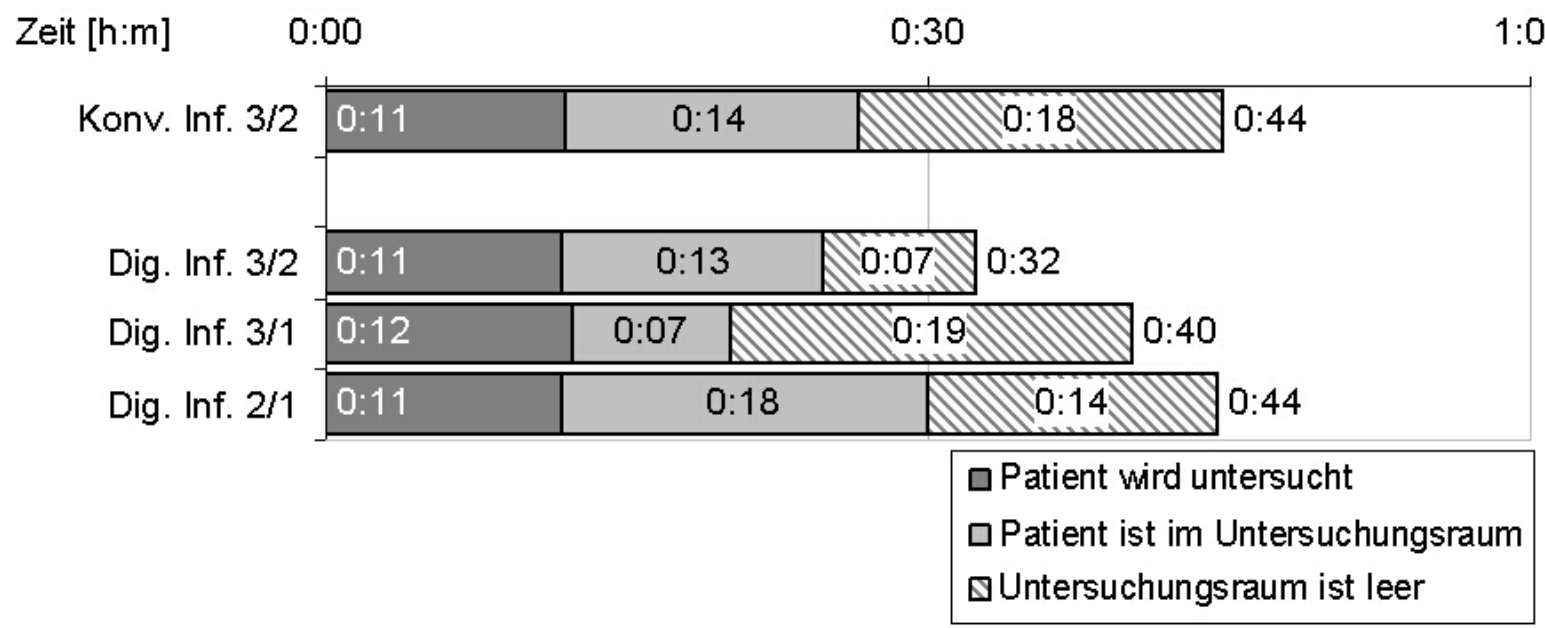

Abbildung 5-5: Geräteauslastung pro Untersuchung mit Gerätetaktzeit. Gegenüberstellung eines Szenariums mit konventioneller Infrastruktur und mehrerer Szenarien mit digitaler Infrastruktur. Unterschiedliche personelle Besetzung (Ärzte/MTRAs).

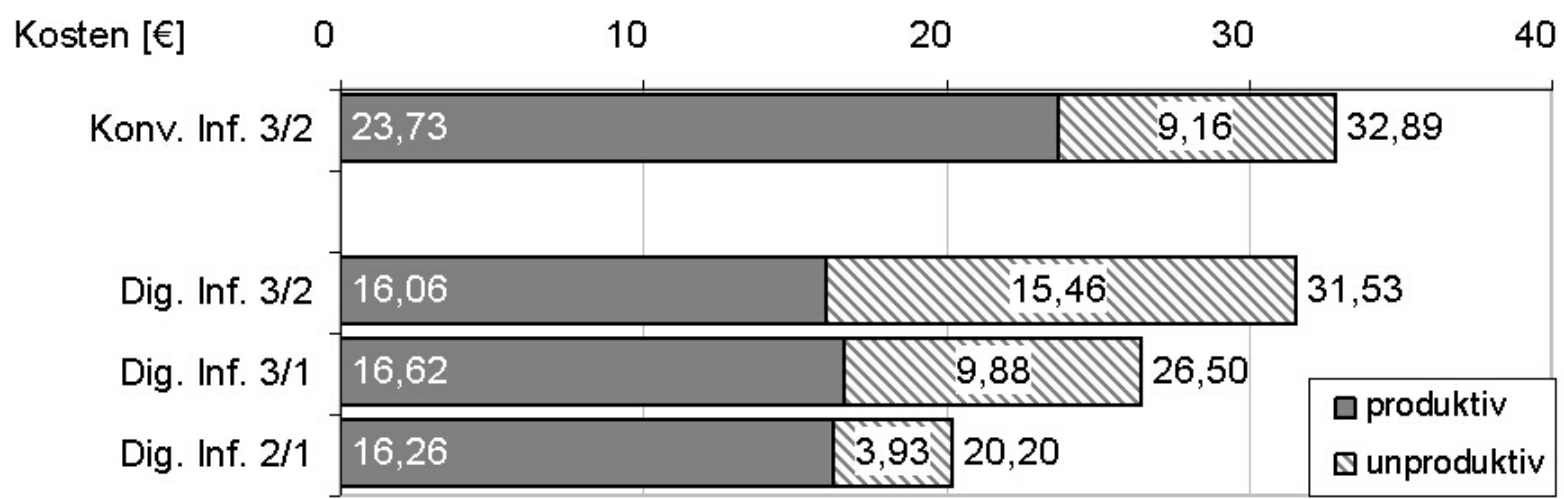

Abbildung 5-6: Produktive und unproduktive Personalkosten pro Untersuchung. Gegenüberstellung eines Szenariums mit konventioneller Infrastruktur und mehrerer Szenarien mit digitaler Infrastruktur. Unterschiedlicher personelle Besetzung (Ärzte/MTRAs).

\subsection{Diskussion}

Mitarbeiter und Entscheidungsträger in radiologischen Kliniken assoziieren zum 
Begriff der elektronischen Infrastruktur in erster Linie das PACS. In der Annahme, dass durch die Umstellung vom filmbasierten Arbeiten und Archivieren auf PACS Kosten allein durch Materialeinsparungen reduziert werden, haben sich viele Kliniken ein PACS angeschafft. Häufig wird dann fest gestellt, dass die Gesamtkosten steigen. Ursache hierfür sind in erster Linie die zusätzlichen Mensch-MaschineSchnittstellen, die auftreten, wenn das PACS die bestehenden Arbeitsabläufe lediglich ergänzen soll. Erst wenn neben der elektronischen Speicherung und Verarbeitung auch die Möglichkeiten der Automatisierung von Abläufen konsequent genutzt werden, entfaltet eine elektronische Infrastruktur ihr Einsparungspotential. So konnte mit einer über 10 Jahre eingeführten klinikübergreifenden digitalen Infrastruktur im Baltimore Veterans Affairs Medical Center der Prozess des ThoraxRöntgens erheblich vereinfacht werden. Die Anzahl der Arbeitsschritte wurde von 52 auf 11 reduziert (87). Ein ähnlicher Trend ist im Prozessmodell zu erkennen: Die Anzahl der Arbeitsschritte wurde für das Szenarium einer digitalen Infrastruktur von 29 auf 14 reduziert. Eine Reduktion des Arbeitsaufwands ist hieraus zwar abzuleiten, jedoch wurden bisher mangels vorhergehender Messungen keine Angaben zum quantitativen Ausmaß der Aufwandsverminderung und damit zur Höhe der einzusparenden Kosten gemacht. Die Simulation erlaubt dagegen eine quantitative Abschätzung der zu erwartenden Einsparungen. Die Aussagekraft der Simulation steht und fält mit dem Vertrauen, dass die simulierten Daten mit den realen Daten übereinstimmen (78). Unterständiger Rückkopplung der prozessbeteiligten Ärzten und MTRAs wurde die strukturelle Gültigkeit des Modells wiederholt verifiziert. Zudem mussten die simulierten Daten mit den gemessenen Daten auf mehreren Ebenen übereinstimmen. Insgesamt wurde das Modell als ausreichend valide für die gegebene Fragestellung beurteilt.

Der simulierte Nettoarbeitsaufwand pro Untersuchung bei digitaler Infrastruktur reduzierte sich gegenüber der konventionellen Infrastruktur um 44,2\% von 0:52 h auf 0:29 h. Umgerechnet auf die Bruttopersonalkosten ergibt sich eine Reduktion um $38,6 \%$ von $32,89 €$ auf $20,20 €$. Diese Reduktion wird über die Einsparung von Personal erzielt, da die Untersuchungen jetzt von zwei Ärzten und einem MTRA durchgeführt werden. Die Durchlaufzeit der Patienten steigt dabei gegenüber dem konventionellen Szenarium um 15,1\% von 0:52 h auf 1:00 h an. Gegenüber der für inn schnelleren Lösung mit drei Ärzten und zwei MTRAs bei digitaler Infrastruktur $(0: 45 \mathrm{~h})$ besteht sogar ein Anstieg um 32,1\%. Hier werden allerdings keine Kosten 
gespart, während die Ressourcenauslastung sinkt. Eventuell besteht auch die Möglichkeit, die Anwesenheitsdauer durch andere Optimierungsmaßnahmen zu reduzieren. Die Anwesenheitszeit der Patienten nimmt bei einem MTRA gegenüber zweien leicht ab. Erwarten würde man den umgekehrten Trend. Der Effekt ist auf eine Eigenheit des Prozessmodells zurück zu führen. Die Tätigkeit „Patient abrufen“ hat eine niedrige Priorität. Ein Patient wird nur abgerufen, wenn ein MTRA gerade nichts Wichtigeres zu tun hat. Dieser Zustand tritt häufiger auf, wenn mehrere MTRAs zur Verfügung stehen.

Die Aufwandsreduktion betrifft ausschließlich den administrativen Anteil der Untersuchung. Der Arbeitsaufwand am Patienten bleibt hier unberührt, da die einzelnen Vorgangsdauern gleich blieben. Dies kann sich ändern, wenn z.B. im Vorgang $1 \mathrm{j}$ (Befund erstellen und drucken) statt der Befundeingabe per Tastatur ein Spracherkennungssystem zum Einsatz kommt $(76,93)$. Generell sind Verkürzungen überall dort zu erwarten, wo Mensch-Computer-Schnittstellen effizienter gestaltet werden. Bisher werden z.B. die Stammdaten des Patienten immer wieder neu am Ultraschallgerät und am Befundungscomputer eingetippt. Hier ist ein Effizienzgewinn zu erwarten, wenn die Geräte automatisiert auf einen gemeinsamen Datenpool zugreifen

können.

Alle Personaleinsparungen vor Ort müssen in Relation gesehen werden zu zusätzlichen Personal- und sonstige Kosten, die Einrichtung und Betrieb einer digitalen Infrastruktur mit sich bringen. Legt man die ermittelten Werte zugrunde, kann an dem einen Arbeitsplatz mindestens ein MTRA weniger beschäftigt werden, während der Patientendurchsatz sich erhöht. Das bedeutet eine jährliche Einsparung von $39790 €$ (Haushaltsbrutto) bei steigenden Einnahmen. Eine digitale Infrastruktur bedient alle Arbeitsplätze einer Klinik und macht viele zusätzliche Aufgaben wie Botengänge oder integritätserhaltende Aktenarbeit überflüssig. Bei entsprechender Anpassung des Arbeitsablaufs birgt sie trotz hoher Investitions- und zusätzlicher Betriebskosten ein immenses Potential zur Effizienzsteigerung und Kosteneinsparung. 


\section{Anhang \\ 6.1 Literatur}

\section{Literaturverzeichnis}

1. DIN 69900-1: Projektwirtschaft, Netzplantechnik, Begriffe. 1 Berlin, Beuth-Verlag, 1987

2. Sonographie-Honorar deckt nicht einmal die Kosten. Trotzdem keine höhere Bewertung?. Medical Tribune. 1994, 24, S.51-52,

3. Continuous Quality Improvement. University of Alabama, 2002

4. Process Win9x, ME, NT, 2000, XP. sciforma corporation, 2002

5. Stat.Fit. Win 9x, ME, NT, 2000, XP. South Kent, Connecticut, USA, Geer Mountain Software Corp., 2002

6. Andrews PJ, Piper IR, Dearden NM, Miller JD: Secondary Insults During Intrahospital Transport of Head Injured Patients.. Lancet. 1990, 335, S.327$330,8685$.

7. Attebo L, Bengtsson $M$, Johnson A: Comparison of Portable Emergency Ventilators Using a Lung Model.. Br J Anesth. 1993, 70, S.372-377, 3.

8. Banks J: Principles of Simulation. Banks J. 1998, S.3-30,

10. Bredenberg CE, lannettoni M, Rosenbloom M, Hodge CJ, Litvin GK, Aust JC: Operative Angiography by Intraarterial Digital Subtraction Angiography: A New Technique for Quality Control ofCarotid Endarterectomy. J Vasc Surg. 1989, 9, S.530-534, 4.

9. Block L: Project Management - New Characters, New Results. Hosp Prog. 1973, 54, S.68-71, 5.

11. Brye PE, Loharikar R, Duda E: New Picture Archiving and Communications System Plus New Facility Equals Critical Path Planning Challenge.. J Digit Imag. 1999, 12, S.130-133,

12. Buffa ES: Modern Production Management.. 3 New York, Wiley, 1969

13. Coffey RJ, Othman JE, Walters JI: Extending the Application of Critical Path Methods.. Qual Manag Health Care. 1995, 3, S.14-29,

14. Coffey RJ, Richards JS, Remmert CS, LeRoy SS, Schoville RR, Baldwin PJ: An Introduction to Critical Paths.. Qual Manag Health Care. 1992, 1, S.45-54,

16. Cono M, Dawson KA: Determining the Size of the Gastroenterology Divison Expansion Using Simulation: A Case Study. Proc Annual HIMSS Conf. 1993, 2, S.127-137,

15. Cohen EL: Nursing Case Management: Does it Pay?. J Nurs Admin. 1991, 21, S.20-25,

17. D'Alesandro JB: Process Simulation as a Total Quality Management Tool.. Production Modeling Corporation, 2002

18. D'Aquila NW: Facilitating in Service Programs through PERT/CPM.. J Nurs Manag . 1993, 24, S.92-94,

19. Dale BG, Lascelles DM, Plunkett JJ: The Process of Total Quality Management.. 1990 S.3-18, 
21. Diplock C, de Vulder J: Better Project Management Through Network Planning.. Health Soc Serv J. 1979, 89, S.B47-B51,

20. Dawson KA, Ulgen OM, O'Connor K, Sanchez P: How to Conduct a Successful Emergency. Band Center Staffing Simulation Study. Production Modeling Corporation., 2002

22. Doncaster REmploying Modern Quality Improvement Principles to Enhance Performance Resulted in a Comprehensive Service Metrics Program a Massachusetts General Hospital's Radiology Department.Imag Economics19991118-26

23. Dowd MT, Hathaway PB, Fontaine AB, Borsa JJ, Nelsen M, Hoffer E: Combined Use of Portable CT and Fluoroscopy in the Angiography Suite.. Am J Radiol. 1999, 11/12, S.18-26,

24. Ehrenwerth J, Sorbo S, Hackel A: Transport of Critically III Adults.. Crit Care Med. 1986, 14, S.497-498,

25. Eichhorn S: Profitcenter-Organisation und Prozessorientierung.. Stuttgart, Berlin, Köln, Kohlhammer, 1999

26. Eisenberg JM: Doctors' Decision and the Cost of Medical Care.. Health Administrations Press Perspectives, 1986

27. Etheredge ML: Colaborative Care: Nursing Case Management.. 1993, 19, S.8-16,

30. Farley K: The COPD Critical Pathway: a Case Study in Progress.. Qual Manag Health Care. 1995, 3, S.43-54,

32. Frehr HU: Total Quality Management.. München, Carl Hansen Verlag, 1993

31. Feldstein PJ, Wickizer TM, Wheeler JR: Private Cost Containment. The Effect of Utilization Review Programs on HEalth Care Use and Expenditures.. Engl J Med. 1988, 318, S.1310-1314,

33. Gaitanides M, Scholz R, Vrohling A: Prozessmanagement - Umsetzung und Zielsetzungen. Prozessmanagement - Konzepte, Umsetzungen und Erfahrungen des Reengineering.. München, Carl Hansen Verlag, 1994 S.1-19,

34. Gaitanides M, vrohlings A, Scholz R: Prozeßmanagement - Umsetzungen und Erfahrungen des Reengineering.. München, Wien, Carl Hansen, 1994 S.13,

35. Statistical Validation of Simulation Models.. La Jolla, California, Simulation Councils, 1974

36. Gerhardt P: Diagnostic Radiology in the Cost Development Spectrum in Medicine.. Radiologe. 1996, 36, S.270-278,

37. Gervais HW, Eberle B, Konitzke D, Hennes HJ, Dick W: Comparison of Blood Gases of Ventilated Patients During Transport.. Crit Care Med. 1987, 15, S.761-763,

38. Gil Corrales MA: The PERT-CPM Method in Health Programming.. Salud Publica Mex. 1971, 13, S.953-966,

40. Giuliano KK, Poirier CE: Nursing Case Management: Critical Pathways to Desirable Outcomes.. Nurs Manag. 1991, 22, S.52-55,

39. Gillessen C, Lehmkuhl LHJ, Teichgräber UK: Softwaretool zur Zeitlichen Erfassung parallel ablaufender Arbeitsvorgänge für die Prozessanalyse in einer Radiologischen Abteilung.. Gesundheitsökonomie und Qualitätsmanagement. 2002, 1-4, 
41. Greene JH: Production and Inventory Control: Systems and Decicions.. Homewood, IL, 1974 American Hospital Publishing, 1989

28. Ethridge P, Lamb GS: Professional Nursing Case Manegement Improves Quality, Access and Costs.. J Nurs Manag. 1989, 20, S.30-35,

29. Falconer: The Critical Pathway Method in Stroke Rehabilitation: Lessons from an Experiment in Cost Containment and Outcome Improvement.. Qual RevBull.

42. Gunnarsson T, Theodorsson A, Karlsson P, Fridriksson S, Bostrom S, Persliden $\mathrm{J}$ : Mobile Computed Tomography Scanning in the Neurosurgery Intensive Care Unit: Increase in Patient Safety and Reduction of Staff Workload. J Neurosurg. 2000, 93, S.432-436,

43. Gwinn R, Cleary K, Medlock M: Use of a Portable CT Scanner during Resection of Subcortical Supratentorial Astrocytomas of Childhood.. Pediatr Neurosurg. 2000,32, S.37-43,

44. Hansen DA: Total Quality Management (TQM). Tutorial Help Page, AT\&T Worldnet, 2002

45. Hehir MD, Hollands MJ, Deane SA: The Accuracy of the First Chest X-Ray in the Trauma Patient.. Aust NZJ Surg. 1990, 60, S.529-532,

46. Hofmann PA: Critical Path Method: an Important Tool for Coordinating Clinical Care.. J Qual Improv. 1993, 19, S.235-246,

48. Jolliet P, Slosman DO, Ricou B, Suter PM, Chevrolet JC: Pulmonary Scintigraphy at the Bedside in Intensive Care Patients with Suspected Pulmonary Embolism.. Int Care Med. 1995, 21, S.723-728,

47. Hurst JM, Davis K, Johnson DJ, Branson RD, Branson PS: Cost and Complications During in-Hospital Transport of Critically III Patients: a Prospective Cohort Study.. J Trauma. 1992, 33, S.582-585,

49. Jones RH: PERT/CPM Network analysis: a Management Tool for Hospital Pharmacists Involved in Strategic Planning.. Hosp Pharm. 1984, 19, S.89-90,

50. Karcz A: Critical Pathways to Utilization Review.. Adm Radiol. 1995, 14, S.23-25,

51. Kost GJ: Applicatio of Program Evaluation and Review Technic (PERT) to laboratory Research and Development Planning.. Am J Clin Pathol. 1986, 86, S.186-192,

52. Laux A, Rogalla P, Kern H, Hamm B: Use of a Portable CT Scanner for Monitoring Ventilated Patients in an Intensive Care Unit.. RoFo Fortschr Geb Rontgenstr Neue Bildgeb Verf. 2001, 173, S.591-594,

53. Lockvet KGJ: Critical Path Analysis and Other Project Network Techniques.. 5 London, Pitman Publishing, 1991

54. London J: On the Right Path. Colaborative Case Management Makes Nurses Partners in the Care-Planning Process.. Health Progress. 1993, 74, S.36-38,

56. Luttman RJ, Laffel GL, Pearson SD: Using PERT/CPM (Program Evaluation and Review Technique/Critical Path Method) to Design and Improve Clinical Processes.. Qual Manag Health. 1995, 3, S.1-13,

55. Lord J: Practical Strategies for Implementing Continuous Quality Improvement.. Manag Care Q. 1993, 1, S.43-52,

57. Lutz H, Hansemann M: Anhaltszahlen für Ultraschalldiagnostik.. Ultraschall in der Medizin. 1985, 6, S.351, 
58. Matson MB, Jarosz JM, Gallacher D, Malcolm PN, Holemans JA, Leong C: Evaluation of Head Examinations Produced with a Mobile CT Unit. Br J Radiol. 1999, 72, S.631-636,

59. Mattox DE, Mirvis SE: Intraoperative Portable Computed Tomography Scanning: An Adjunct to Sinus and Skull Base Surgery.. Otolaryng Head Neck Surg. 1999, 121, S.776-780,

60. McGuire F: Simulation in Healthcare.. New York, John Wiley \& Sons, Inc, 1998 S.605-627,

61. McQuillan PJ, Hilman DR, Woods WP: Positive End Expiratory Pressure and Critical Oxygenation during Transport in Ventilated Patients.. Int Care Med. 1990, 16, S.513-516,

62. Moder T, Phillips F, Davis J: Project Management with CPM/PERT and Precedence Diagramming.. New York, Van Norstrand Reinhold, 1983

63. Morris R, Macken L, Tail G: Failure of Exhalation During Ventilation with the Drager Oxylog 2000 Ventilator.. Anesth Int Care. 1999, 27, S.400-404,

64. Mosher C, Cronk P, Kidd A, McCormick P, Stockton S, Sulla C: Upgrading Practice with Critical Pathways.. Am J Nurs. 1992, 92, S.41-44,

65. Müller R: Röntgendiagnostik pulmonaler Komplikationen nach allogener Knochenmarktransplantation - Anwendung und Erfahrung mit der digitalen Lumineszenzradiographie.. Pulmologie. 1991, 45, S.977-986,

66. Neumann K, Steinhardt U: GERT Networks and the Time-Oriented Evaluation of Projects.. Berlin Heidelberg, Springer Verlag, 1979

67. Neuwied: Gesundheitspolitik im Zeitalter chronischer Erkrankungen.. Band Das moderne Krankenhaus. Luchterhand, 1994 S.95-105,

68. Oess A: Total Quality Management - die ganzheitliche Qualitätsstrategie.. 1991 S.89,

69. Parry MH: Project Management - what is it?. Can Hosp. 1973, 50, S.42-43,

70. Pearson SD, Goulart-Fisher D, Lee TH: Critical Pathways as a Strategy for Improving Care: Problems and Potential.. Ann Int Med. 1995, 123, S.941-948,

71. Pinkernelle JG, Teichgraber UK, Born C, Ricke J, Felix R: Einsatz der mobilen Computertomographie bei nicht transportfähigen patienten. RoFo Fortschr Geb Rontgenstr Neuen Bildgeb Verfahr . 2002, 174, S.747-753,

72. Reuß J, Weiss H, Wanner T, Leser HJ,: Zeitaufwand von ärztlichen und nichtärztlichen Mitarbeitern für Ultraschalluntersuchungen.. Ultraschall in der Medizin. 1998, 19, S.126-129,

73. Rhea JT: Longterm improvement in cost an quality within hospitals.. Hospital and Health Services Administration. 1986, 31, S.64-73,

75. Rhea JT, Thrall JH, Saini S, Sumner J: Improving the efficiency and service of computed tomographic scanning.. Academic Radiology. 1994, 1, S.164-170,

76. Rosenthal DF, Bos JM, Sokolowski RA, Mayo JB, Quigley KA, Powell RA: A voice-enabled, structures medical reporting system.. Am J Med Inform Assoc. 1997, 4, S.436-441,

77. Ryan JE: Project management teams smooth organizational change.. Hosp Financ Manage. 1979, 33, S.26-27, 
Farrington PA, Nembhard HB, Sturrock DT, Evans GW: Validation and Verification of Simulation Models.. 1999

79. Schriefer J: Managing Critical Pathway Variances.. Qual Manag Health Care. 1995, 3, S.30-42,

80. Schwarze J: Projektmanagement mit Netzplantechnik.. Herne/Berlin, Neue Wirtschaftsbriefe $\mathrm{GmbH}, 2001$

81. Schwarze J: Eine Einführung in das Projektmanagement. 7 Herne/Berlin, Verlag Neue Wirtschaftsbriefe, 1994

82. Seifert K: Leitmotiv "Kundenorientierung" vs. Bürgerorientierung. Prozeßmanagement für die öffentliche Verwaltung.. Wiesbaden, Deutscher Universitätsverlag, 1998 S.160-163,

83. Seifert K: Operationalisierung der Prozessqualität. Prozessmanage,ent für die öffentliche Verwaltung.. Wiesbaden, Deutscher Universitätsverlag, 1998 S.210-212,

85. Seifert K: Prozeßmanagement für die öffentliche Verwaltung.. Wiesbaden, Deutscher Universitätsverlag, 1998 S.228f.,

86. Shoemaker WC: Critical Path Medicine.. Crit Care Med. 1974, 2, S.279,

84. Seifert K: Prozessmanagement - Eine ganzheitliche Optimierungsphilosphie. Prozeßmanagement für die öffentliche Verwaltung.. Wiesbaden, Deutscher Universitäts Verlag, 1998 S.158-160,

87. Siegel E, Reiner B: Work Flow Redesign: The Key to Success when Using PACS.. Am J Radiol. 2002, 178, S.563-566,

88. Simon H, Homburg C: Kundenzufriedenheit, Konzepte- Methoden- Erfahrungen. 1995 S.211f,

89. Smith I, Fleming S, Cernaianu A: Mishaps during Transport from the Intensive Care Unit.. Crit Care Med. 1990, 18, S.278-281,

90. Teichgräber UK, Pinkernelle J, Born C, Barckow D, Bittner RC: Mobile Computertomographie im Routinebetrieb auf einer Intensivstation.. Medica Mundi. 2001, 1, S.45-48,

91. Teichgräber UK, Benter T, Klühs L, Schröder RJ, Hidajat N, Dörken B: Netzplantechnik zur Zeitplanung bei abdominellen Ultraschalluntersuchungen.. Ultraschall in der Medizin. 1999, 20, S.236-241,

93. Teichgräber UK, Ehrenstein T, Ricke J, Felix R: Speech Recognition Helps Eliminate Errors.. Diagnostic Imaging Europe. 2001, 10, S.21-25,

92. Teichgräber UK, Ehrenstein T, Lemke M, Liebig T, Stobbe H, Hosten N: Digitale Spracherkennung bei der Erfassung computertomographischer Befundtexte.. RoFo Geb Rontgenstr Neuen Bildgeb Verfahr. 1999, 171, S.396-399,

94. Teichgräber UK, Neumann F, Boeck J, Ricke J, Felix R: Process Management in Computed Tomography: Using Critical Pathway Method to Design and Improve Work Flow in Computed Tomography.. Eur Radiol. 2000, 10, S.370376,

95. Teichgräber UK, Pinkernelle JG, Ricke J, Jürgensen JS, Barckow D, Felix R: Aggressive Use of Diagnostic Imaging on the Intensive Care Unit.. Int Care Med. 2001, 27, S.75,

96. Wagner HM: Principles of Operations Research.. Englewood Cliffs, NJ, Prentice- 
Hall, 1975

97. Weber C, Maas R, Steiner P, Kramer J, Bumann D, Zander AR: Wertigkeit der digitalen Thoraxaufnahme bei der Detektion von Lungeninfiltraten knochenmarktransplantierter Patienten in der Aplasie.. RöFo Fortschr Geb Röntgenstr Neuen Bildgeb Verfahr. 1999, 171, S.294-301,

98. Weingarten SR, Riedinger MS, Conner L, Lee TH, Hoffmann I, Johnson B: Practice Guidelines and Reminders to Reduce Duration of Hospital Stay with Chest Pain. An Interventional Trial.. Ann Int Med. 1994, 20, S.257-263,

99. Wennberg JE, Blowers L, Parker R, Gittelsohn AM: Changes in Tonsillectomy Rates Associated with Feedback and Review.. Pediatrics. 1977, 59, S.821826 ,

100. White CS, Meyer CA, Wu J, Mirvis SE: Portable CT: Assessing Thoracic Disease in the Intensive Care Unit.. Am J Radiol. 173, S.1351-1356,

101. Whitehouse GE: Systems Analysis and Design Using Network Techniques.. Prentice Hall, 1973

102. Wickizer TM: The Effects of Utilization Review on Hospital Use and Expenditures: A Covariance Analysis.. Health Serv Res. 1992, 27, S.103-121,

104. Zander K: Nursing Case Management: Strategic Management of Cost and Quality Outcomes.. J Nurs Admin. 1988, 5, S.23-30,

105. Zander K: Focusing on Patient Outcome: Case Management in the 90s. Dimens Crit Care Nurs. 1992, 11, S.127-129, 


\subsection{Abkürzungsverzeichnis}

A

AA

Abkürzung

$A E$

ARDS

AUDI

BAT

bzw.

ca.

$\mathrm{cm}$

CPM

CT

d.h.

DEGUM

Dig.

DIN

DRG

DSA

EA

EE

EF

EF

ES

Fa.

FAZ

FP

GAN

GERT

GP

$\mathrm{H}$

HA

$H D$

IAPP

Inf.
Arbeitsaufwand

Anfang-Anfang

Bedeutung

Anfang-Ende

Adult Respiratory Distress Syndrome

Aggressive Use of Diagnostic Imaging

Bundesangestellten Tarif

beziehungsweise

Circa

Zentimeter

Critical Pathway Method

Computertomographie

das heißt

Deutsche Gesellschaft für Ultraschall in der Medizin

Digital

Deutsche Industrienorm

Diagnoses Related Groups

Digitale Substraktionsangiographie

Ende-Anfang

Ende-Ende

Early finish

Endfolge

Early start

Firma

frühester Anfangszeitpunkt

freie Pufferzeit

Generalized Activity Networks

Graphical Evaluation and Review Technique

Gesamtpufferzeit

Stunde

Auftretenshäufigkeit

häufigste Dauer

Intra Arterial Pulmonal Pressure

Information 
kg

Konv.

KV

LS

$\min$

MPM

MTRA

MW

N

NF

NPT

OD

OP

P

P

PACS

PD

PERT

R

RIS

Robsys

$\mathrm{S}$

s.u.

sec

SF

T

$\mathrm{t}$

Tab.

UP

US

USA

z.B.

ZE
Kilogramm

konventionell

Kassenärztliche Vereinigung

Late Start

Minute

Metra Potential Method

Medizinisch technische Röntgenassistentin

Mittelwert

Anzahl der Messwerte

Normalfolge

Netzplantechnik

optimistische Dauer

Operation / Operationssaal

Patientendurchlaufzeiten

Signifikanzniveau

Picture archiving and communication system

pessimistische Dauer

Program Evaluation and Review Method

Ressourcenauslastung

Radiologisches Informations- und Abrechnungssystem

Radiologisches Organisations- und Befundsystem

Sekunde

siehe unten

Sekunde

Sprungfolge

Taktzeiten

Zeit

Tabelle

unabhängige Pufferzeit

Ultraschall

Vereinigte Staaten von Amerika

zum Beispiel

Zeiteinheiten 


\subsection{Abbildungsverzeichnis}

Abbildung 1-1: Zusammenhang von Komplexität, Aufwand und Nutzen für den

Anwender bei Anwendung von Prozessmanagementmethoden.

Abbildung 1-2: CPM/ PERT Netzplan einer CT-Untersuchung unter optimistischen

Bedingungen. Kritischer Weg in schwarz markiert. ........................................... 16

Abbildung 3-1: Gantry mit Beatmungseinheit .............................................. 21

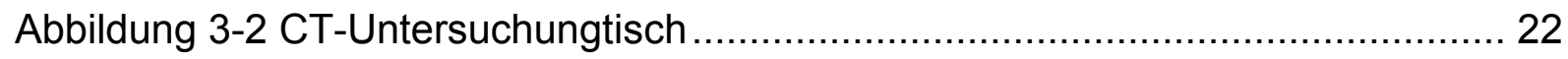

Abbildung 3-3: CT-Computerkonsole (Steuereinheit) ..................................... 22

Abbildung 3-4: Grundriss des Interventionsraums auf der Intensivstation ............... 23

Abbildung 3-5: Gantt-Diagramm mit dem Arbeitsablauf zur Durchführung einer mobilen Computertomographie im Interventionsraum der Intensivstation. .............. 26

Abbildung 3-6: Transferzeiten von den Patientenzimmern zu den

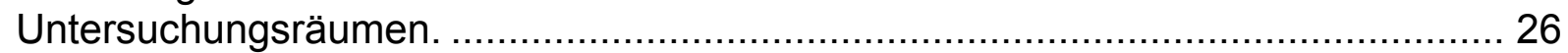

Abbildung 3-7: Zeitdauer der Topogrammerstellung und der Bildakquisition............ 27

Abbildung 3-8: Zeit-Entfernungskorrelation für den Transport eines Patienten von der

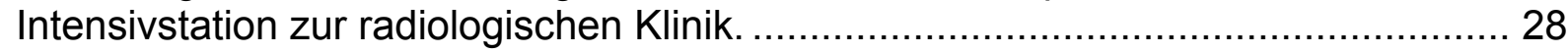

Abbildung 4-1: Beschreibung der Daten eines Arbeitschrittes im Netzplan............. 37

Abbildung 4-2: Beziehungsschema der Arbeitsschritte der abdominellen

Ultraschalluntersuchung ( $X=$ Vorgänge (Arbeitsschritte), $S=$ Scheinvorgänge) ...... 39

Abbildung 4-3: Strukturplan abdominelle Ultraschalluntersuchung....................... 41

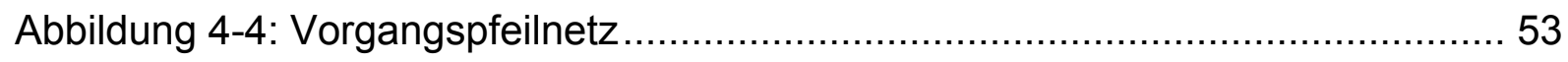

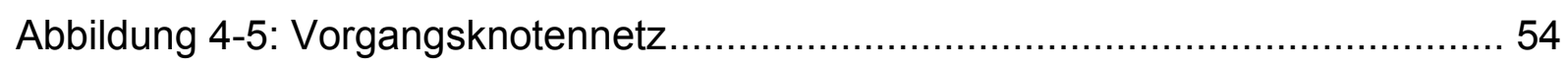

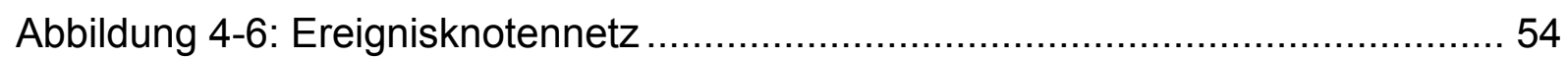

Abbildung 4-7: Gemischtorientierter Netzplan .............................................. 54

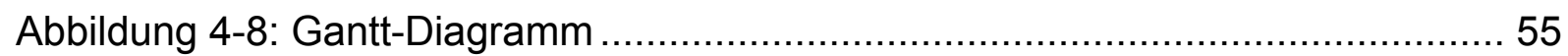

Abbildung 4-9: Schematische Darstellung eines Netzplans mit Scheinvorgang

(gestrichelte Linie) .................................................................................... 56

Abbildung 4-10: Metra-Potential-Skizze .................................................. 57

Abbildung 4-11: Bedienungsoberfläche der proprietären Software zur Zeitmessung 62

Abbildung 4-12: Funktionsbereich 1, optimistische Betrachtung .......................... 66

Abbildung 4-13: Funktionsbereich 1, realistische Betrachtung .............................. 66

Abbildung 4-14: Funktionsbereich 2, realistische Betrachtung .............................. 66

Abbildung 4-15: Planungs- und Durchführungs- und Überprüfungszyklus für einen PERT/CPM Netzplan.

Abbildung 4-16: Schematische Darstellung der Erstellung von PERT Diagrammen

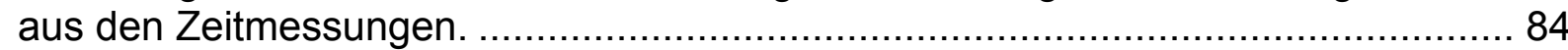

Abbildung 4-17: Verteilung der erfassten Untersuchungen ............................... 89

Abbildung 4-18: Vorgang 3g - Untersuchung durchführen (CT S, CT Thorax, mobile Patienten: n=32). Anzahl der gemessenen Zeitdauern. OD: Optimistische Dauer; HD: Häufigste Dauer; PD: Pessimistische Dauer. Werte in Minuten. .......................... 91

Abbildung 4-19: Realistischer Netzplan am CT S, CT Thorax, mobile Patienten. 
Kritische Vorgänge in schwarz; Abschnitte in grau (I: Vorbereitung; II: Durchführung; III: Nachbereitung); Nicht kritische Vorgänge in weiß; Gesamte Pufferzeit nach nichtkritischen Vorgängen in grau. Zeit in Minuten.

Abbildung 4-20: Vergleich der Durchführungsdauer bei unterschiedlichen Patientenkategorien; M: mobile Patienten; L: bettlägerige Patienten; I: Intensivpatienten (CT S, CT Thorax).

Abbildung 5-1: Flussdiagramm der Prozessstruktur; Evaluationsmodell. Die grau unterlegten Vorgänge dienen der Prozesssteuerung. Prozessstruktur: Vom Evaluationsprozess wurden alle ausbildungsorientierten Vorgänge („1i“ und Steuervorgänge, s. Abbildung 4-1 bis 4-3) entfernt.

Abbildung 5-2: Validierung der Prozessdaten, Vergleich der gemessenen Patientenlaufzeiten von Patientenregistrierung (Aktivität 1c) bis Nachbereitung der Untersuchung (Aktivität 1I) mit den simulieren Zeiten in Abhängigkeit von Tageszeit

Abbildung 5-3: Prozessstruktur mit digitaler Infrastruktur

Abbildung 5-4: Arbeitsaufwand für Untersuchungs- und Administrationstätigkeiten sowie unproduktive Zeiten pro Untersuchung. Gegenüberstellung eines Szenariums mit konventioneller Infrastruktur und mehrerer Szenarien mit digitaler Infrastruktur. Unterschiedliche personelle Besetzung (Ärzte/MTRAs).

Abbildung 5-5: Geräteauslastung pro Untersuchung mit Gerätetaktzeit.

Gegenüberstellung eines Szenariums mit konventioneller Infrastruktur und mehrerer Szenarien mit digitaler Infrastruktur. Unterschiedliche personelle Besetzung (Ärzte/MTRAs).

Abbildung 5-6: Produktive und unproduktive Personalkosten pro Untersuchung. Gegenüberstellung eines Szenariums mit konventioneller Infrastruktur und mehrerer Szenarien mit digitaler Infrastruktur. Unterschiedlicher personelle Besetzung (Ärzte/MTRAs). 


\subsection{Tabellenverzeichnis}

Tabelle 1-1: Auswirkungen und Vorteile durch die Anwendung von Netzplantechnik

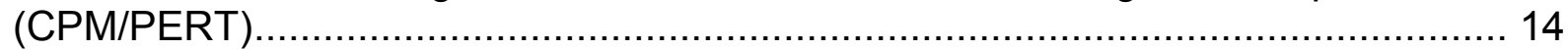

Tabelle 1-2: Erstellung eines Netzplans in vier Phasen ................................. 15

Tabelle 4-1: Arbeitsschritte der abdominellen Ultraschalluntersuchung ................... 38

Tabelle 4-2: Netzplantechniken im Vergleich. (IOR: Inclusive OR. IOR ist WAHR, wenn aus einer Menge von Bedingungen eine oder mehrere erfüllt sind. EOR:

Exclusive OR. EOR ist WAHR, wenn aus einer Menge von Bedingungen genau eine erfüllt ist.)

Tabelle 4-3: Auflistung der Arbeitsvorgänge mit Vorgänger und

Ressourcenallokation (Funktionsbereich 1; (to: optimistische Zeit , $t_{r}$ : realistische Zeit, $t_{p}$ : pessimistische Zeit))

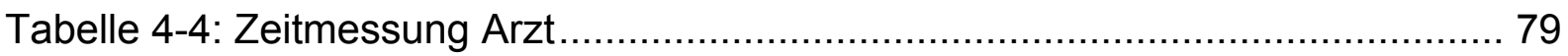

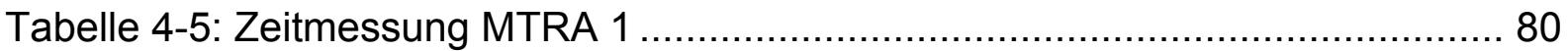

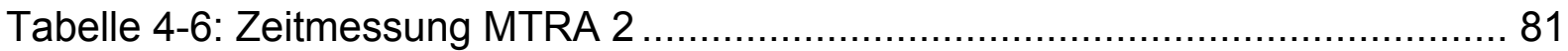

Tabelle 4-7: Untersuchungszahlen an den CT-Geräten unterteilt nach

Untersuchungsarten für das Jahr 1999

Tabelle 4-8: Vorgänge des Radiologen: PERT (CT S, CT Thorax, mobile Patienten: $n=32)$. n(V): Auftretenshäufigkeit der Vorgänge; RD: realistische Dauer; OD: optimistische Dauer; PD: pessimistische Dauer.

Tabelle 4-9: Vorgänge der MTRA 1: PERT (CT S, CT Thorax, mobile Patienten: $n=32) . n(V)$ : Auftretenshäufigkeit der Vorgänge; RD: realistische Dauer; OD: optimistische Dauer; PD: pessimistische Dauer. Zeiten in Minuten.

Tabelle 4-10: Vorgänge der MTRA 2: PERT (CT S, CT Thorax, mobile Patienten: $n=32) . n(V)$ : Auftretenshäufigkeit der Vorgänge; RD: realistische Dauer; OD: optimistische Dauer; PD: pessimistische Dauer, Zeiten in Minuten).

Tabelle 4-11: Vorgänge des Radiologen; CPM (CT S, CT Thorax, mobile Patienten). FAZ: Frühester Anfangszeitpunkt; SAZ: Spätester Anfangszeitpunkt; FEZ: Frühester Endzeitpunkt; SEZ: Spätester Endzeitpunkt; FP: Freie Pufferzeit; GP: Gesamte Pufferzeit. Zeiten in Minuten.

Tabelle 4-12: Vorgänge der MTRA 1: CPM (CT S, CT Thorax, mobile Patienten); FAZ: Frühester Anfangszeitpunkt; SAZ: Spätester Anfangszeitpunkt; FEZ: Frühester Endzeitpunkt; SEZ: Spätester Endzeitpunkt; FP: Freie Pufferzeit; GP: Gesamte Pufferzeit. Zeiten in Minuten.

Tabelle 4-13: Vorgänge der MTRA 2: CPM (CT S, CT Thorax, mobile Patienten); FAZ: Frühester Anfangszeitpunkt; SAZ: Spätester Anfangszeitpunkt; FEZ: Frühester Endzeitpunkt; SEZ: Spätester Endzeitpunkt; FP: Freie Pufferzeit; GP: Gesamte Pufferzeit. Zeiten in Minuten. ................................................................. 100

Tabelle 5-1: Erstellung eines Prozessmodells in fünf Phasen ..............................113

Tabelle 5-2: Indikatoren der Prozessleistung. .115

Tabelle 5-3: Ermittelte Vorgänge einer Ultraschalluntersuchung mit Beschreibung, Ressourcen, Vorgängern, Auftretenshäufigkeit $\left(\mathrm{H}_{\mathrm{A}}\right)$, Anzahl der Messwerte $(\mathrm{n})$, Mittelwert der Messwerte $(\mu \mathrm{MW})$, Mittelwert der simulierten Werte ( $\mu$ Sim.) und Signifikanzniveau (p). 


\section{5}

\section{Danksagung}

Danken möchte ich an erster Stelle Herrn Professor Dr. med. Dr. h.c. Roland Felix, ohne dessen kontinuierliche Unterstützung und Diskussionsbereitschaft diese Arbeit nicht möglich gewesen wäre.

Weiterhin schulde ich Herrn Christoph Gillessen, Herrn Fabian Neumann und Herrn Jens Pinkernelle Dank, die durch ihre konstruktive Zusammenarbeit wesentlich zum Gelingen der Arbeit beigetragen haben.

Bei den Mitarbeitern der Klinik für Strahlenheilkunde möchte ich mich für die stete Unterstützung für meine Arbeit bedanken.

Herrn James Rhea, M.D. Associate Professor of Radiology und Herrn Robert A. Novelline, M.D. Professor of Radiology aus dem Massachusetts General Hospital and Harvard Medical School bin ich für die Einführung in den Themenbereich des Prozessmanagements dankbar. 


\section{6}

\section{Eidesstattliche Versicherung}

Gemäß Habilitationsordnung der Medizinischen Fakultät Charité

Hiermit erkläre ich, dass

- keine staatsanwaltlichen Ermittlungsverfahren gegen mich anhängig sind,

- weder früher noch gleichzeitig ein Habilitationsverfahren durchgeführt oder angemeldet wurde bzw. welchen Ausgang ein durchgeführtes Habilitationsverfahren hatte;

- die vorgelegte Habilitationsschrift ohne fremde Hilfe verfasst, die beschriebenen Ergebnisse selbst gewonnen wurden, sowie die verwendeten Hilfsmittel, die Zusammenarbeit mit anderen Wissenschaftlerinnen oder Wissenschaftlern und technischen Hilfskräften und die Literatur vollständig angegeben sind,

- mir die geltende Habilitationsordnung bekannt ist. 